BONPLANDIA 18(2): 95-189. 2009

\title{
LAS RAZAS DE MANÍ DE BOLIVIA
}

\author{
ANTONIO KRAPOVICKAS ${ }^{1}$, RICARDO O. VANNI ${ }^{2}$, JOSÉ R. PIETRARELLI ${ }^{3}$, DAVID E. WILLIAMS ${ }^{4} \&$ CHARLES \\ E. SIMPSON 5
}

\begin{abstract}
Krapovickas, A., R. O. Vanni, J. R. Pietrarelli, D. E. Williams \& C. E. Simpson. 2009. The peanut landraces from Bolivia. Bonplandia 18(2): 95-189. ISSN: 0524-0476.

Bolivia is regarded as the probable place of origin of the domesticated peanut, and an important world center of unique peanut diversity. As the first published study of its kind on peanut, this paper documents the infraspecific diversity of the crop in its country of origin and center of diverstity. 62 distinct landraces of Bolivian peanut were identified and systematically described. 42 landraces belong to Arachis hypogaea L. ssp. hypogaea var. hypogaea; 17 to $A$. hypogaea ssp. fastigiata var. fastigiata; one to $A$. hypogaea ssp. fastigiata var. vulgaris; and two to $A$. hypogaea ssp. fastigiata var. peruviana. With very few exceptions, the landraces encountered in Bolivia are almost entirely endemic to that country. The most typical peanuts from Bolivia pertain to the landraces "Crema", "Colorado San Simón", "Bayo americano", "Overo", and "Overo carenado", which are widely cultivated throughout the country. A few regions of unusually high peanut diversity can be identified. In the Yungas region of La Paz, 11 landraces were collected, of which three are endemic. In the mountainous regions of Santa Cruz and Cochabamba, 18 landraces were collected, of which six are endemic. The Department of Tarija yielded 14 landraces, of which two are endemic. All of the aforementioned landraces pertain to the botanical variety hypogaea. In contrast, the subspecies fastigiata has a remarkable center or diversity in the watershed of the Rio Beni, where 10 landraces were collected in a fairly small area, nine of which are endemic to that region. This monograph is intended to enhance the knowledge and appreciation of peanut diversity, and facilitate the conservation and use of peanut landraces by scientistis, plant breeders, and farmers.
\end{abstract}

Key words: Arachis hypogaea, biodiversity, cultivated races, peanut.

Resumen: Krapovickas, A., R. O. Vanni, J. R. Pietrarelli, D. E. Williams \& C. E. Simpson. 2009. Las razas de maní de Bolivia. Bonplandia 18(2): 95-189. ISSN: 0524-0476.

Bolivia se destaca como probable lugar de origen del maní cultivado y un centro de variación único en el mundo, lo que señala a Bolivia como un importante fuente de materiales para el mejoramiento de este cultivo de importancia mundial En este estudio para maní se diferencian para Bolivia 62 razas de maní cultivado de las cuales, 42 pertenecen a Arachis hypogaea $\mathrm{L}$ subsp.hypogaea var. hypogaea, 17 a $A$. hypogaea susbp. fastigiata Waldron var. fastigiata, 1 a $A$. hypogaea susbp. fastigiata var. vulgaris y 2 a $A$. hypogaea subsp. fastigiata var. peruviana Krapov. \& W. C. Gregory. Bolivia constituye un centro de variación independiente pues sus razas son exclusivas de su territorio, salvo muy pocas excepciones. Bolivia como unidad, se

${ }^{12}$ Instituto de Botánica del Nordeste (UNNE-CONICET), Casilla de Correo 209, 3400 Corrientes, Argentina.

${ }^{3}$ Estación Experimental INTA Manfredi, Córdoba, Argentina.

${ }^{4}$ System-wide Genetic Resources Programme (SGRP), c/o Bioversity International, Rome, Italy.

${ }^{5}$ Texas \& University, Stephenville, Texas, USA. 


\begin{abstract}
caracteriza por las razas "Crema", "'Colorado San Simón", '"Bayo americano", "'Overo" y "Overo carenado", que se cultivan en todo el país. Se pueden delimitar algunas regiones de mayor diversidad: la región de los Yungas de La Paz, donde se coleccionaron 11 razas de las cuales 3 son exclusivas y la región montañosa de Santa Cruz y Cochabamba, con 18 razas de las cuales 6 son exclusivas. Estas dos regiones pertenecen a la cuenca del Amazonas. Una tercera región se encuentra en el Departamento de Tarija, perteneciente a la cuenca del Plata, donde se coleccionaron 14 razas, de las cuales 2 son exclusivas. Todas estas razas pertenecen a la var. hypogaea. La subsp. fastigiata, presenta un centro de variación muy notable en la cuenca del río Beni, donde en un área muy pequeña se coleccionaron 10 razas, de las cuales 9 son exclusivas. Se destaca Bolivia como un centro de variación único en el mundo y la gran variabilidad del maní señala a Bolivia como una importante fuente de posibilidades para el mejoramiento de este cultivo.
\end{abstract}

Palabras clave: Arachis hypogaea, biodiversidad, razas cultivadas, maní.

\section{Introducción}

En Sudamérica, salvo contadas excepciones, el maní no es un producto de gran importancia económica, sin embargo está ampliamente difundido y se lo encuentra cultivado por todas partes en las zonas cálidas del continente. En la mayoría de los casos, es un cultivo hortícola, para uso familiar y de venta en pequeña escala.

El análisis de las amplias colecciones de germoplasma de maní reunidas a partir de 1959, muestran una regionalización de las razas locales muy manifiesta. Ecuador y Perú comparten muy pocas razas entre sí. Entre Ecuador, Perú y Bolivia conocemos solamente 3 razas en común. Los maníes de Acre (Brasil) se diferencian tanto de los peruanos como de los bolivianos a pesar de que colinda con éstos dos países. A su vez, los maníes cultivados por los Nambiquara de Rondonia y Mato Grosso incluyen razas o especies únicas. Los maníes cultivados por los Xingú en Mato Grosso se separan también del resto de los sudamericanos. En la región guaranítica, que abarca Paraguay y áreas vecinas de Brasil y Argentina, se cultivan razas, en su mayor parte también exclusivas.

Esta diferenciación refleja una acción selectiva humana muy prolongada en el tiempo, debido a la importancia que siempre tuvo el maní en la dieta, a causa de sus buenas características nutritivas.

Se ha podido sistematizar la variabilidad del maní, estableciendo una jerarquía taxonómica, en buena parte confirmada por electroforesis (Grosso \& al., 1988, 1994). En Sudamerica estos taxones infraespecíficos presentan centros de variación genética independientes y geograficamente diferenciados.

Se postula que Bolivia es el país de origen del maní cultivado (Arachis hypogaea L.) debido a su alta diversidad de razas primitivas y por la ocurrencia de las especies silvestres de Arachis más cercanamente emparentadas con el cultígeno. Hasta 1954 había muy poca o ninguna información sobre los maníes de Bolivia. Por ejemplo, en 'Le marché mondial des arachides" (Anónimo, 1954), Bolivia aparece en blanco en los mapas en los que se ilustra la dispersión y la producción del maní en el mundo.

El Ministerio de Asuntos Agropecuarios (1974) dice lo siguiente: "Después de estudiar aproximadamente cien variedades procedentes de diversos lugares, se han seleccionado: "Perla de Saavedra", "Tatuí 76", "Argentino", "Star", "Overo", "Chiquitano", "Colorado", "San Simón", "Tanash", "WC-5", "'Virginia Bunch 67" (Muñoz Reyes, 1980: 315). De éstas once sólo cuatro parecen ser autóctonas: "Overo", "Chiquitano", "Colorado", y "San Simón", el resto corresponde a variedades introducidas de Argentina, Brasil y EE.UU.

La Estación Experimental INTA de Manfredi recibió las primeras muestras de maní originarias de Bolivia en el mes de Julio del año 1946, las que fueron remitidas por Luis Nanetti. La semilla de ese material sembrado en noviembre de ese mismo año, al 
germinar y adquirir el desarrollo vegetativo correspondiente, presentó una serie de formas muy diferentes de las poblaciones existentes en la colección de Manfredi de ese entonces, revelando las mismas portes diferentes, ciclo vegetativo muy largo, para las condiciones climáticas de la región manisera de Córdoba y muy buen comportamiento frente a los ataques de "viruela" (Cercospora spp.).

Estas primeras muestras, así como las coleccionadas posteriormente por A. Krapovickas en 1950 y 1958, pertenecían todas a Arachis hypogaea subsp. hypogaea var. hypogaea. En 1959, el equipo formado por W.
C. Gregory, A. Krapovickas y J. Pietrarelli, coleccionó en la zona de influencia de Santa Cruz de la Sierra las primeras muestras bolivianas pertenecientes a $A$. hypogaea subsp. fastigiata Waldron var. fastigiata.

En el mes de marzo de 1977 comenzaron las prospecciones (Gregory, 1977, 1979; Pietrarelli, 1961, 1968, 1982; Simpson, 1980, 1982, 1984; Williams, 1989a, 1989b, 1991a, 1991b, 1996) cuyo equipo estaba integrado por técnicos de Argentina, EE.UU. y Bolivia. Esas exploraciones y recolecciones de muestras prosiguieron en los años 1979 y 1980.

En octubre de 1981, el equipo Pietrarelli-

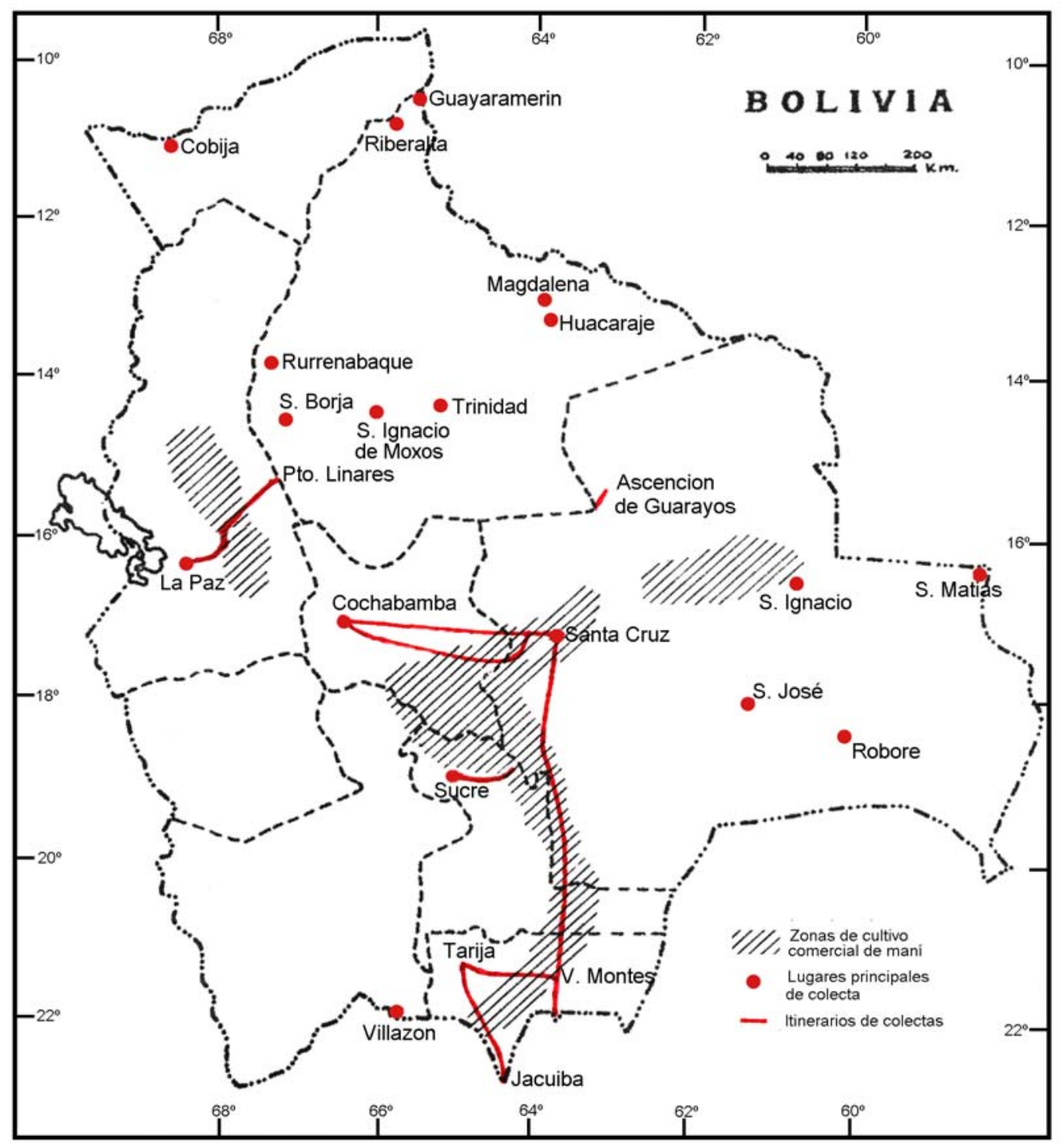

Fig. 1. Itinerarios y lugares de colección de muestras de maní (mapa base tomado de Muñoz Reyes, 1980). 
Zanini, ambos de la EEA de Manfredi, con fondos del IBPGR (FAO), encontró una gran variabilidad de plantas y con diferentes colores de tegumento de las semillas, en la región del Beni, exactamente en las márgenes del río del mismo nombre. Todas ellas pertenecientes a la subsp. fastigiata var. fastigiata. Posteriormente y en diferentes períodos D. E. Williams, por entonces botánico del New York Botanical Garden, incrementó esa colección con muestras de La Paz, Beni y Pando, incluyendo algunas de A. hypogaea subsp. fastigiata var. peruviana.

En la actualidad la colección de Manfredi asciende a unas 700 accesiones de Bolivia que abarcan gran parte de las zonas aptas para el cultivo del maní en dicho país.

Las colecciones se hicieron en chacras, mercados y centros de acopio. Muchas de las muestras obtenidas estaban constituidas por mezclas de colores. Por ejemplo, en Entre Ríos (Tarija), la colección US 826 contenía las razas "Colorado San Simon", "Colorado carenado", "Overo", Overo tarijeño", "Overo carenado" y "Overo cojín carenado", y la US 832 contenía las razas "Sopachuy", "Overo", "Overo alazán", "Churco" y "Colorado San Simon".

Estas mezclas explicarían en parte, la gran variabilidad de los maníes bolivianos por favorecer la posibilidad de cruzamientos espontáneos provocados por la visita de insectos. El material original coleccionado fue repartido entre Manfredi, USDA, ICRISAT e instituciones bolivianas, incluyendo CIAT-Saavedra, y el Centro de Investigaciones Fitoecogenéticas de Pairumani.

En este estudio se pudieron diferenciar para Bolivia 62 razas de maní cultivado de las cuales: 42 pertenecen a A. hypogaea subsp. hypogaea L. var. hypogaea.

17 a A. hypogaea subsp. fastigiata Waldron var. fastigiata.

1 a A. hypogaea subsp. fastigiata var. vulgaris C. Harz y 2 a A. hypogaea subsp. fastigiata var. peruviana Krapov. \& W.C. Gregory.

Bolivia constituye un centro de variación independiente pues sus razas son endémicas de su territorio, salvo muy pocas excepciones. Con Paraguay y Brasil, en la región guaranítica, presenta en común únicamente una raza de la var. hypogaea, el "Crema" que es idéntico al "Guaycurú", que se cultiva a lo largo del Alto Paraná y que se extiende hasta el nordeste de Brasil.

Con Perú, las razas en común son: el "Rastrero veteado", coleccionado en Sud Yungas y en Cuzco (Perú), perteneciente a la var. hypogaea, junto con el "Taba" y el "Panza", pertenecientes a la var. peruviana, el primero coleccionado en Cobija (Pando), el segundo en Rurrenabaque (Beni). La única muestra obtenida de la var. vulgaris es un remanente de anteriores introducciones comerciales. Con el desarrollo agrícola de Santa Cruz de la Sierra y de Villa Montes (Tarija) se introdujeron algunas razas brasileñas como el "Tatú" y también algunos maníes tipo "Spanish" (var. vulgaris), provenientes de Taiwán, que no prosperaron.

El límite de altitud del cultivo del maní en Bolivia, es de aproximadamente $2000 \mathrm{~m}$. s.n.m. La subsp. hypogaea, con razas típicamente tardías, se cultiva principalmente en zonas montañosas, a excepción del "Overo" y el "Crema", que se extienden por todo el territorio boliviano. En cambio, la subsp. fastigiata, que es más precóz, es cultivada exclusivamente en las llanuras bajas orientales.

En la subsp. hypogaea hay razas con caracteres únicos, sólo presentes en Bolivia. En el resto del mundo hay sólo dos portes de planta: tipo "Virginia Runner" y tipo "Virginia Bunch". En Bolivia en cambio, hay razas gigantes, que denominamos tipo cojín, de hasta 1,50 m de diámetro y 60-70 cm de altura.

Los tipos "Virginia" tienen uniformemente 2 granos por caja, pero en Bolivia son frecuentes razas de la subsp. hypogaea con hasta 3 o 4 granos y cajas mucho más grandes.

Fuera de Bolivia, los overos son muy poco frecuentes y siempre son colorados y albos. En cambio, en Bolivia los overos muestran una variabilidad muy grande de colores y asociaciones: colorado, rosado, salmón, alazán y albo.

Los progenitores silvestres de Arachis hypogaea: A. duranensis Krapov. \& W.C.Gregory y A. ipaensis Krapov. \& W.C.Gregory (Krapovickas, 2004; Seijo \& al, 2004) viven en la cercanía de Villa Montes (Tarija), son de porte rastrero, con frutos muy pequeños y dos granos por fruto. Es llamativo 
que la única raza rastrera con los frutos más pequeños y con dos granos sea el "Rastrero colorado de dos granos", proveniente también de Villa Montes.

Bolivia como unidad se caracteriza por las razas 7."'Crema", 29.'"Colorado San Simón", 38." Bayo americano", 41."Overo" y 42." Overo carenado", que se cultivan en todo el país.

Se pueden distinguir algunas regiones por su alta divesidad. Una delimitada por las localidades de Samaipata y Vallegrande (Santa Cruz) y Mizque (Cochabamba), donde se han coleccionado 18 razas, de las cuales 6 son exclusivas.Una segunda región se encuentra en el Departamento de Tarija, donde se coleccionaron 14 razas de las cuales 2 son exclusivas y una tercera en los Yungas de La Paz, donde se coleccionaron 11 razas, de las cuales 3 son exclusivas. Todas estas razas pertenecen a la var. hypogaea.

En cuanto a la subsp. fastigiata, hay un centro de variación muy notable en la cuenca del río Beni. En un área muy pequeña se han coleccionado las siguientes razas:

43."Colorado precoz" San Borja

49." Colorado de Cero-Ocho" Yacuma

51." "Colorado de Rurrenabaque" Rurrenabaque

53."Colorado cáscara negra"

54." "Pálido del Beni"

55."Pálido de Uchupiamonas"

56." Negro de Rurrenabaque"

58."Amarillo"

59."Albo"

62."Panza"

La gran variabilidad del maní en Bolivia, señala a este país como un centro de variación único en el mundo y por lo tanto representa una importante fuente de posibilidades para el mejoramiento de este cultivo.

\section{Materiales y Métodos}

El estudio aquí presentado para la clasificación de las razas de maní de Bolivia se llevó a cabo durante sucesivos ciclos experimentales realizados entre 1960 y 2007, en la Estación Experimental Agrícola Manfredi (EEA Manfredi), ubicada en el centro de la franja manicera de la provincia de Córdoba, Argentina (31 $48^{\prime} \mathrm{S}, 63^{\circ} 40^{\prime} \mathrm{W}$ alt. 290 m s.n.m.). Se trabajo con unas 700 entradas de maní de origen boliviano que forman parte de la gran colección de germoplasma de Arachis mantenida por INTA en la EEA Manfredi, en la cual se conservan más de 2000 accesiones de maní de más de 20 paises, de los cuales 1200 son originarios de la región andina (Bolivia, Perú y Ecuador).

Uno de los desafíos que se enfrentó para la clasificación de las razas de maní es el hecho de que posee fructificación subterránea, porque cuando se analizan los frutos ya no existen las plantas y viceversa. Para superar este inconveniente, se registraron cuidadosamente las características agromorfológicas de las plantas y de los frutos en sus respectivas temporadas idóneas, durante repetidos ciclos y posteriormente se juntó esta información de manera sistemática para lograr una clasificación práctica basada en el conjunto de dichas características.

En Manfredi, se registraban las accesiones según su lugar de origen, nombre de los coleccionistas y se les asignaba un número de registro RCM o US. El material fue ordenado en primer término por el sistema de ramificación, el porte, el color del grano y la forma del fruto o caja.

La colección se sembró en el campo según las afinidades morfológicas. Por ejemplo, todos los "Overos" juntos, todos los "Crema" también juntos, etc.

Se trató de uniformar las parcelas, separando las plantas fuera de tipo. De esta manera la planta que se analizaba representaba a toda la parcela.

Este ordenamiento permitió apreciar pequeñas diferencias y afinidades que de otra manera serían difíciles de notar. Por ejemplo, al estar juntas todas las parcelas afines, se vió claramente que la subsp. hypogaea tiene un color verde más intenso que la subsp. fastigiata.

De acuerdo a la ramificación, al tipo de caja y número de semillas por fruto, se las separaba en las dos subespecies: hypogaea y fastigiata (Krapovickas \& Rigoni, 1960; Krapovickas \& Gregory, 1994, 2007). A su vez se trataba de separar por variedades. 
Reunida la colección de un país, en este caso Bolivia, a las diferentes accesiones y posibles razas, se las sembraban en parcelas de $10 \mathrm{~m}$ de largo, 2 semillas cada $20 \mathrm{~cm}$. en la misma línea. Cada línea entre sí, separada a $70 \mathrm{~cm}$. entre surco o 1,40 m, dependiendo del tamaño de las plantas. Con menor distancia entre surcos para las plantas de porte erecto de la subsp. fastigiata, y mayor distancia entre surcos para acomodar las plantas rastreras de la subsp. hypogaea.

La planificación de la siembra se realizaba durante el mes de octubre, se procedía a la misma y en el mes de marzo del año siguiente o primeros días de abril, según las lluvias y temperaturas, se realizaba la observación de la colección en el campo para estudiar el tipo de planta (porte, color de los tallos, tipo de ramificación, presencia o ausencia de cerdas, características florales, precocidad, etc.).

En cada parcela se realizaban observaciones. Después de la cosecha, en octubre, se estudiaban los caracteres de las cajas (frutos). A través de los años se fueron uniformando las accesiones, de manera que, las que presentaban similitudes se sembraban en parcelas contiguas.

Para denominar las razas se respetaron los nombres de las muestras. De no ser así, se dieron nombres según la región de cultivo o en otros casos remarcando algún carácter exomorfológico distintivo de la planta, porte, tipo de caja o color del tegumento de la semilla.

La EEA de Manfredi publicó varios catálogos de la colección de maníes en 1959, 1976, 1977/78 (Pietrarelli, 1976, 1977/78) y 1990 (Pietrarelli \& Krapovickas, 1990). En el último catalogo de 1990 figuran los números de parcela de la cosecha 85/86. En el Anexo incluímos estos números para relacionar el presente ordenamiento con el publicado en 1990. También se publicó en Estados Unidos un catálogo abarcando todas las colecciones realizadas hasta ese momento (Simpson \& Higgins, 1984).

En el herbario del Instituto de Botánica del Nordeste (CTES) en Corrientes, se conservan la colección de frutos de maníes de Bolivia y los ejemplares de herbario de la cosecha 85/86.

Los resultados que se presentan aquí son las conclusiones realizadas desde el año 1979 y completadas con la cosecha 2006/7. En esta última se sembraron solo dos accesiones de cada raza y son las que sirvieron para las ilustraciones del presente trabajo.

La formación de este importante banco de germoplasma de maní, fue posible gracias al apoyo de las siguientes instituciones: IBPGR (FAO), USDA (EE.UU.), INTA e IBONE (UNNE-CONICET).

Las siguientes siglas corresponden a los integrantes de los equipos que coleccionaron en Bolivia:

$\mathrm{B}=$ Banks, D. J. USDA, Oklahoma, State University Stillwater Oklahoma, USA.

$\mathrm{Co}=$ Coradin, L. Cenargen/Embrapa, Brasilia, Brasil.

$\mathrm{Cr}=$ Coro, M. Universidad Juan Misael Saracho, Tarija, Bolivia.

F= Fernandez, A. Instituto de Botánica del Nordeste, Corrientes, Argentina.

$\mathrm{G}=$ Gregory, W.C. North Carolina State University, Raleigh, North Carolina, USA.

$\mathrm{Gb}=$ Gibbons, R. W. ICRISAT (International Crops Research Institute for the Semi-Arid Tropics, Patancheru, A.P., India.

Jk= Janicki, L. PRODES, La Paz, Bolivia

K= Krapovickas, A. Instituto de Botánica del Nordeste, Corrientes, Argentina.

Mo= Mroginski, L. A. Instituto de Botánica del Nordeste, Corrientes, Argentina.

$\mathrm{N}=$ Nanetti, L. Bolivia.

$\mathrm{P}=$ Pietrarelli, J. R. INTA (Instituto de Tecnología Agropecuaria), Manfredi, Córdoba, Argentina.

$\mathrm{Pl}=$ Pletikosic, R. Instituto Boliviano de Tecnología Agrícola, Sucre, Bolivia.

$\mathrm{S}=$ Simpson, C. E. Texas \& University, Stephenville, Texas, USA.

Sc $=$ Schinini, A. Instituto de Botánica del Nordeste, Corrientes, Argentina.

$\mathrm{Wi}=$ Williams, D. E. New York Botanical Garden, New York, USA.

Z= Zurita, H. O. CIAT (Centro de Investigación Agrícola Tropical) Santa Cruz de la Sierra, Bolivia

Zi= Zanini, R. H. INTA, Manfredi, Córdoba, Argentina. 


\section{Clave para distinguir los taxones infraespecíficos de A. hypogaea L. de Bolivia}

1. Eje central sin flores y ramas $\mathrm{n}+1$ en las que alternan regularmente dos ramas vegetativas $\mathrm{y}$ dos reproductivas (ramificación alternada). Folíolos oscuros (excepto Rastrero colorado del Beni)

subsp. hypogaea var. hypogaea

$1^{\prime}$. Eje central con flores y ramas laterales en las que las ramas reproductivas y vegetativas no presentan orden (ramificación secuencial). Folíolos claros.

2. Fruto con más de dos semillas. Fructificación extendida.

subsp. fastigiata

3. Frutos con retículo suave o algo marcado, sin que se destaquen las costillas longitudinales. Ramas reproductivas por lo común breves y delgadas.

1. var. fastigiata

3'. Frutos siempre con retículo muy marcado y con costillas longitudinales sobresalientes. Ramas reproductivas largas, 5-10 cm long., robustas, tanto en el eje central como en las ramas laterales.

2. var. peruviana

2’. Frutos por lo común con dos semillas. Fructificación aglomerada hacia la base de la planta. Con frecuencia, espigas compuestas.

3. var. vulgaris

\section{Clave para distinguir las razas de Arachis hypogaea de Bolivia}

\section{Arachis hypogaea subsp. hypogaea var. hypogaea}

1. Granos de un solo color.

2. Plantas rastreras o cojines.

3. Procumbentes, rastreras medianas, hasta 1,20 m de diámetro.

4. Cajas grandes, ca. 4-6 cm long.

5. Cajas hasta $3-4$ granos.

6. Granos colorados.

7. Eje central muy aparente, cajas con retículo marcado, hasta 4 granos.

1. Rastrero Colorado Grande

7’. Eje central no aparente, cajas hasta 3 granos, retículo suave. Folíolos claros.

2. Rastrero Colorado bel Beni

6’ Granos salmón, cajas con retículo grueso diluído.

3. Rastrero Salmón del Beni

5’. Cajas de 2(3) granos. Granos colorados.

8. Cajas con retículo suave y nervaduras logitudinales gruesas.

4.Rastrero de Guayaramerin

8’. Cajas con retículo marcado.

5. Valluno Rastrero

4. Cajas medianas, hasta 3,5 cm long.. 
9. Granos colorados.

\section{Rastrero Colorado De Dos Granos}

9’Granos salmón claro.

7. Crema

9”. Granos negros o violáceos. Brote violáceo.

10. Granos negros.

8. Barcino

10`. Granos violáceos, punta clara.

9. Rastrero Violáceo Punta Clara

3’. Decumbentes, cojínes muy extendidos, 1,40 m de diámetro o más.

11. Eje central muy aparente.

12. Ramas con muchas reproductivas seguidas, basales sinuosas.

10. Chaucha Rosado

$12^{\prime}$.Ramas con secuencia 2V-2R.

13. Cajas muy grandes, hasta 4 granos, colorado clarete. Pl. rastrera.

11. Mani Pico Loro

13’. Cajas de hasta 3 granos, colorados o rosados. Pl. semirastrera a cojín, enmarañado.

12. Yungueño

11'. Eje central poco o no aparente, cojín compacto.

13. Ronco

2'. Cojines grandes.

14. Granos colorados o salmón. Brote verde.

15. Eje central no aparente. Cojín compacto.

16. Cajas reticuladas.

17 Cajas medianas.

18. Semirastrero a cojín grande, muy ramificado. EC algo aparente.

19.Granos salmón.

14. Sopachuy Salmon

19'Granos colorados

15 Sopachuy Colorado

18’. Cojín compacto. EC no aparente.

16. San Jose de Chicaludo

17’. Cajas grandes, hasta (2) 3-4 granos.

20 Granos salmón.

17. Churco

20’Granos colorados.

18. Churco De Tarija

16. Cajas medianas, retículo diluído. Granos colorado fuerte.

19 Cojin Valluno

15’. Eje central aparente, ramas sinuosas. Muy ramificada, hasta N4.

20. Sara Mani 
14’. Granos negros o violáceos. Brote violáceo.

21.Granos negros.

22. Eje central recto.

23. Eje central breve, ramas rectas

$$
\text { 21. Negro Cojin }
$$

23’. Eje central desarrollado, ramas sinuosas.

22. Negro de los Yungas

22’. Eje central sinuoso caído.

23. Negro de Aiquile

21'. Granos morados.

24. Cajas lisas.

24. Morado Mesa Rancho

$24^{\prime}$. Cajas estranguladas.

25. Cojín achatado.

25. Morado de Santa Cruz

25’. Cojín.

26. Chaucha Morado

2". Plantas erectas (bunch).

26. Granos colorados.

27. Hojas verde claro.

27. Colorado Caja Redonda

27'. Hojas verde oscuro.

28. Folíolos revolutos.

28.Colorado Revoluto

28'. Folíolos planos.

29 Ramas rectas.

29 Colorado San Simón

29’. Ramas sinuosas.

30. Colorado Carenado

26’. Granos salmón.

31. Salmón Carenado

1'. Granos bicolores.

30. Plantas rastreras.

31. Granos negro y albo.

31'. Granos alazán y albo.

32. Overo Negro

33. Overo Alazán Rastrero

31". Granos colorado y albo.

32. Granos colorado en manchas.

34. Overo Rastrero 
32`Colorado en líneas.

35. Rastrero Veteado

30’. Cojines grandes, 1,40 m de diámetro .Granos overos, colorado y albo.

36. Overo Cojin Carenado

30”. Cojines compactos, hasta $1 \mathrm{~m}$ de diámetro.

33. Overo fondo rosado.

34. Alazán más rosado.

37. Overo Alazán

34'. Colorado más rosado.

35. Ramas sinuosas. Rosado más rosado claro.

38. Bayo Americano

35’. Ramas rectas.

39. Overo Valluno

33’. Overo colorado fondo albo.

36. Ramas sinuosas.

40. Overo Colorado

$36^{\prime}$. Ramas rectas.

37. Cajas con poca carena o sin ella.

41. Overo

37’. Cajas con carena y pico.

42. Overo Carenado

\section{Arachis hypogaea subsp. fastigiata var. fastigiata de Bolivia}

1. Granos colorados.

2. Plantas, precoces, son las primeras en madurar en la colección.

3. Ramas dísticas (o a penas torcidas en el àpice). Cajas chicas, muchas de 2 granos.

4. Tallo verde. Cajas con carena.

43. Colorado Precoz

4’. Tallo violáceo. Cajas redondas sin carena.

3'. Ramas con filotaxis 2/5.

44. Colorado Distico de Entre Rios

5. Ramas erectas.

6. Cajas con pico y carena.

45.Colorado Menonitas

6’. Cajas sin pico.

7. Cajas con carena.

46. Colorado Precoz Compacto

$7^{\prime}$. Cajas s sin carena.

8. Planta muy ramificada, folíolos normales.

47. Precoz de Villa Montes 
8’. Plantas con pocas ramas al pié, folíolos muy grandes.

5’. Ramas decumbentes.

48. Cartucho

49. Colorado de Cero Ocho

2'. Planta menos precoces, pero más precoces que la var. hypogaea.

9. Cajas de cácascara clara

10. Plantas verde oscuro. Eje central con frecuencia sin flores

50.Cruceño

10'. Plantas verde claro. Eje central con flores.

11. Ramas sinuosas

$11^{\prime}$. Ramas rectas

51. Colorado Rurrenabaque

52. Colorado Picudo

9’. Cajas con cáscara negra

53. Colorado Cascara Negra

1’. Granos pálidos.

12. Cajas con pico

54. Palido del Beni

12’. Cajas sin pico.

55.Palido de Uchupiamonas

1". Granos negros.

1"'. Granos rosado pálido

56. Negro de Rurrenabaque

$1^{\prime \prime \prime}$. Granos amarillos

57. Rosado Palido

58. Amarillo

$1^{\prime \prime \prime \prime}$. Granos albos

59. Albo

Arachis hypogaea L. subsp. fastigiata Wald. var. vulgaris C. Harz.

60. Palido de Villa Montes

Arachis hypogaea L. subsp. fastigiata var. peruviana Krapov. \& W. C. Gregory

1. Granos pálidos

61. Taba

1'. Granos violáceos

62. Panza 


\section{Rastrero colorado grande}

Planta rastrera, hasta 1,20 m de diámetro; eje central muy aparente. Con cerdas hasta en el pecíolo.

Cajas grandes, ca. 4-6 cm long., con retículo marcado, hasta 3 (raro 4) granos. Granos colorados.

06/07 1001 85/86 1701 US 39 GKBSPScZ Colorado grande Sta. Cruz, E.Saavedra

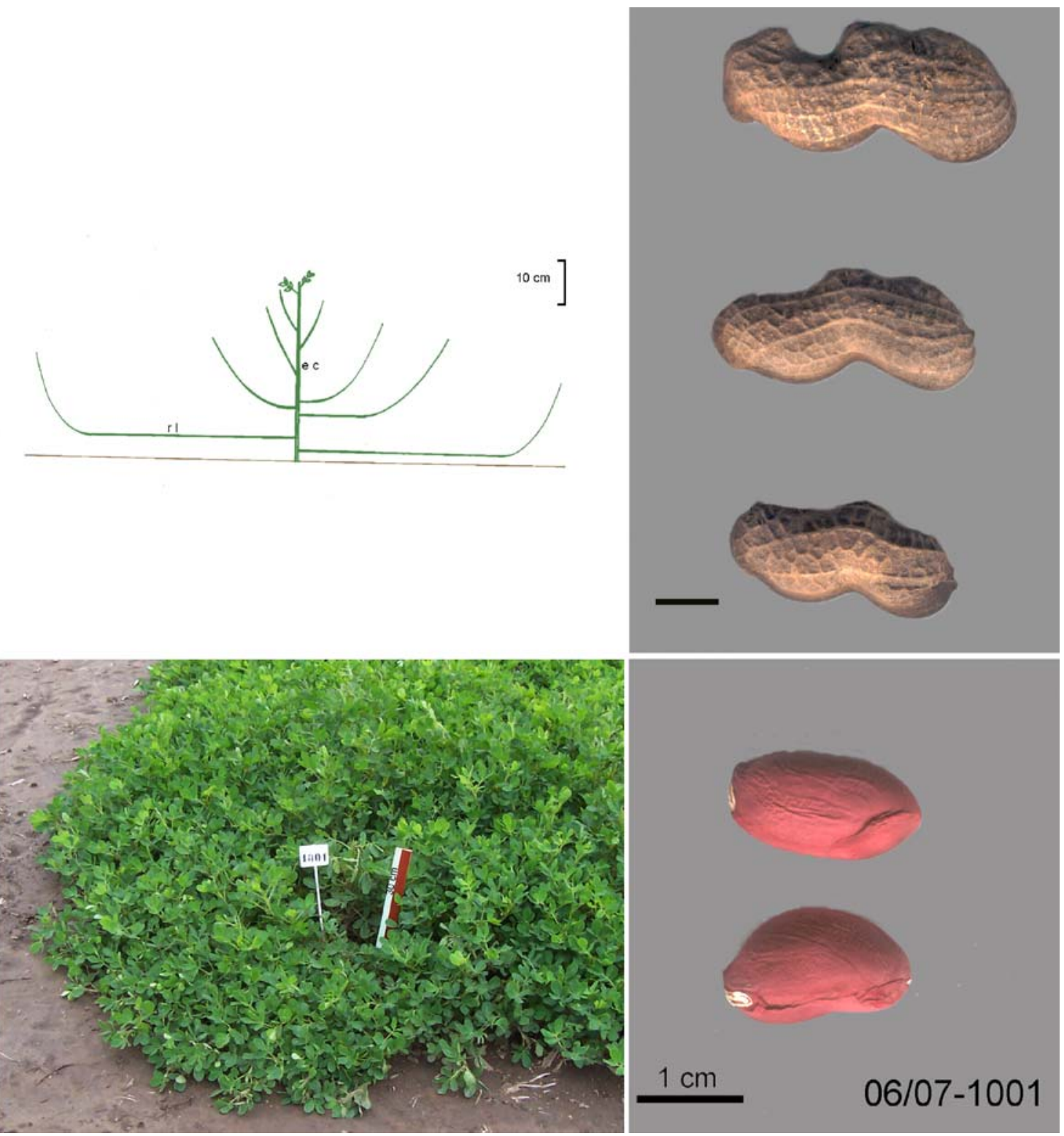

$85 / 861702$

$85 / 861705$

$85 / 861706$

$85 / 861714$
US 45

US 210

US 211

US 345-2
GKBSPScZ Colorado grande GKSPScGb Colorado grande

Colla

KSBScCo
Sta. Cruz, E. A. Saavedra

Sta. Cruz, Sta. Cruz

Sta. Cruz, Sta. Cruz

Sta. Cruz, Sta. Cruz 


\section{Rastrero colorado del Beni}

Planta rastrera compacta, hasta 1,20 de diámetro, eje central no aparente. Con cerdas hasta el pecíolo. Folíolos claros.

Cajas grandes, con retículo más o menos diluído, hasta 3 granos. Granos colorado fuerte.

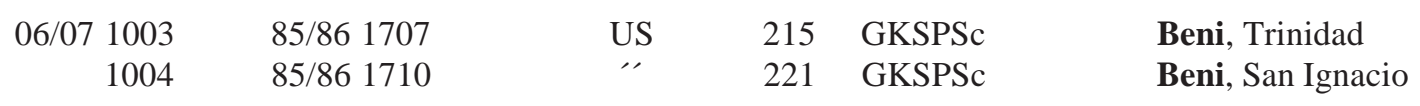
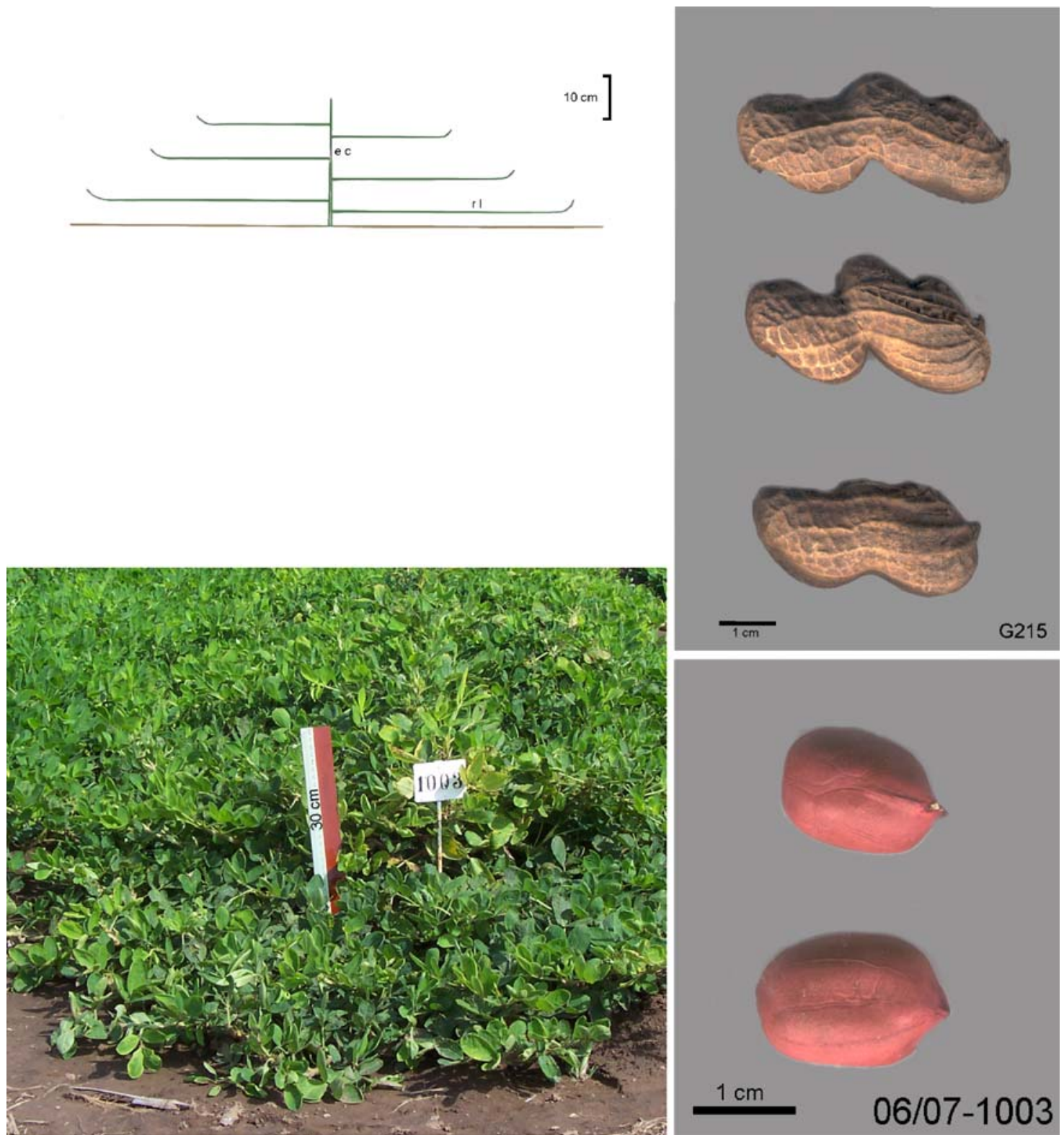


\section{Rastrero salmón del Beni}

Planta rastrera, compacta. Eje central poco aparente. Con cerdas hasta el pecíolo. Folíolos oscuros. Estandarte con aro.

Cajas grandes, con retículo grueso + - diluído, hasta 3 granos. Granos salmón
06/07 1005
US $737 \mathrm{Wi}$
Beni, S. Borja
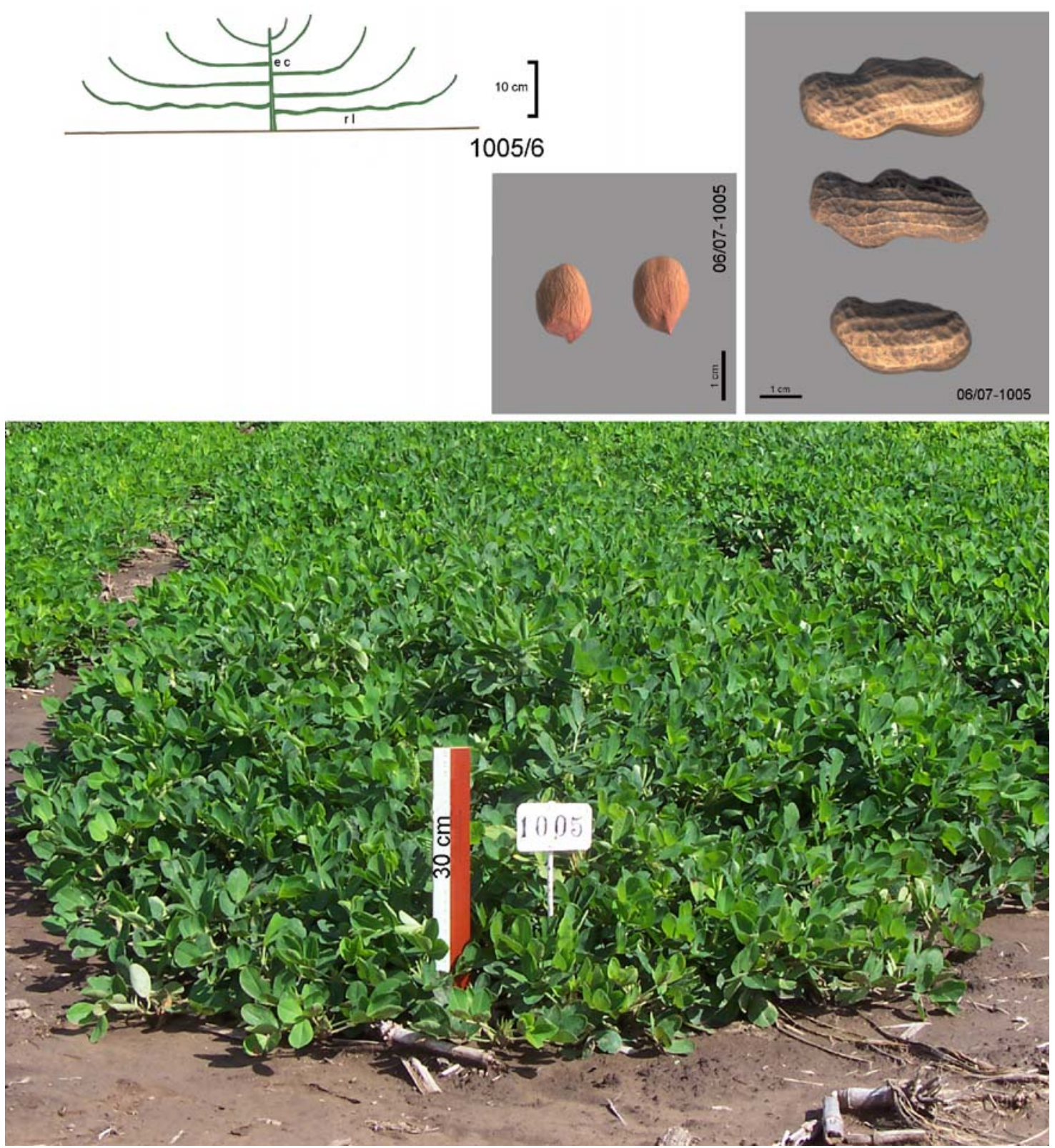

$85 / 861708$

$85 / 861709$

$85 / 861711$
US 215 GKSPSc

US 221

US 397 BZCoJ
Beni, Trinidad

Beni, S. Ignacio-

Alto Beni, S. Ana 


\section{Rastrero de Guayaramerin}

Planta rastrera muy grande, eje central aparente. Cerdas hasta en el raquis.

Cajas grandes, con retículo suave y con nervaduras longitudinales gruesas, 2 (3) granos colorado clarete.
06/07 1007
$85 / 861716$
US 222 GKSPSc
Beni,Guayaramerin
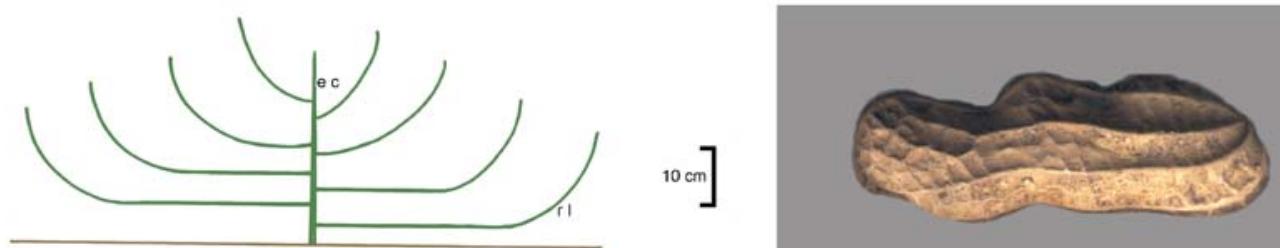

$10 \mathrm{~cm}$

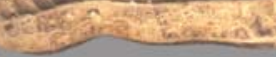

1007
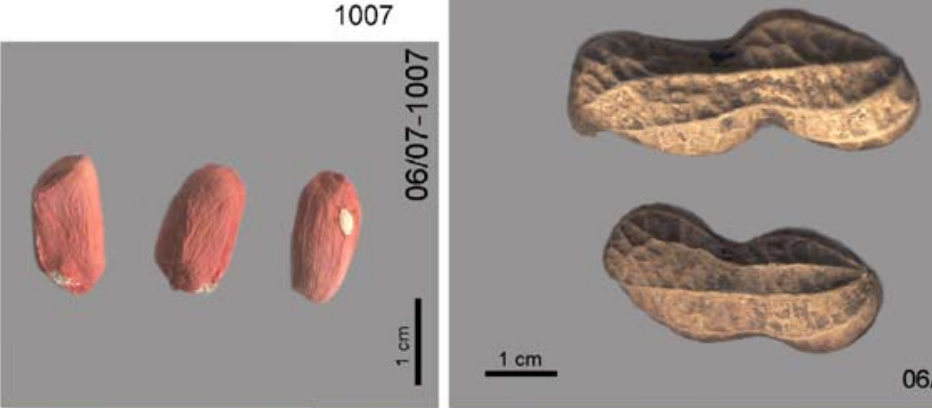

06/07-1007

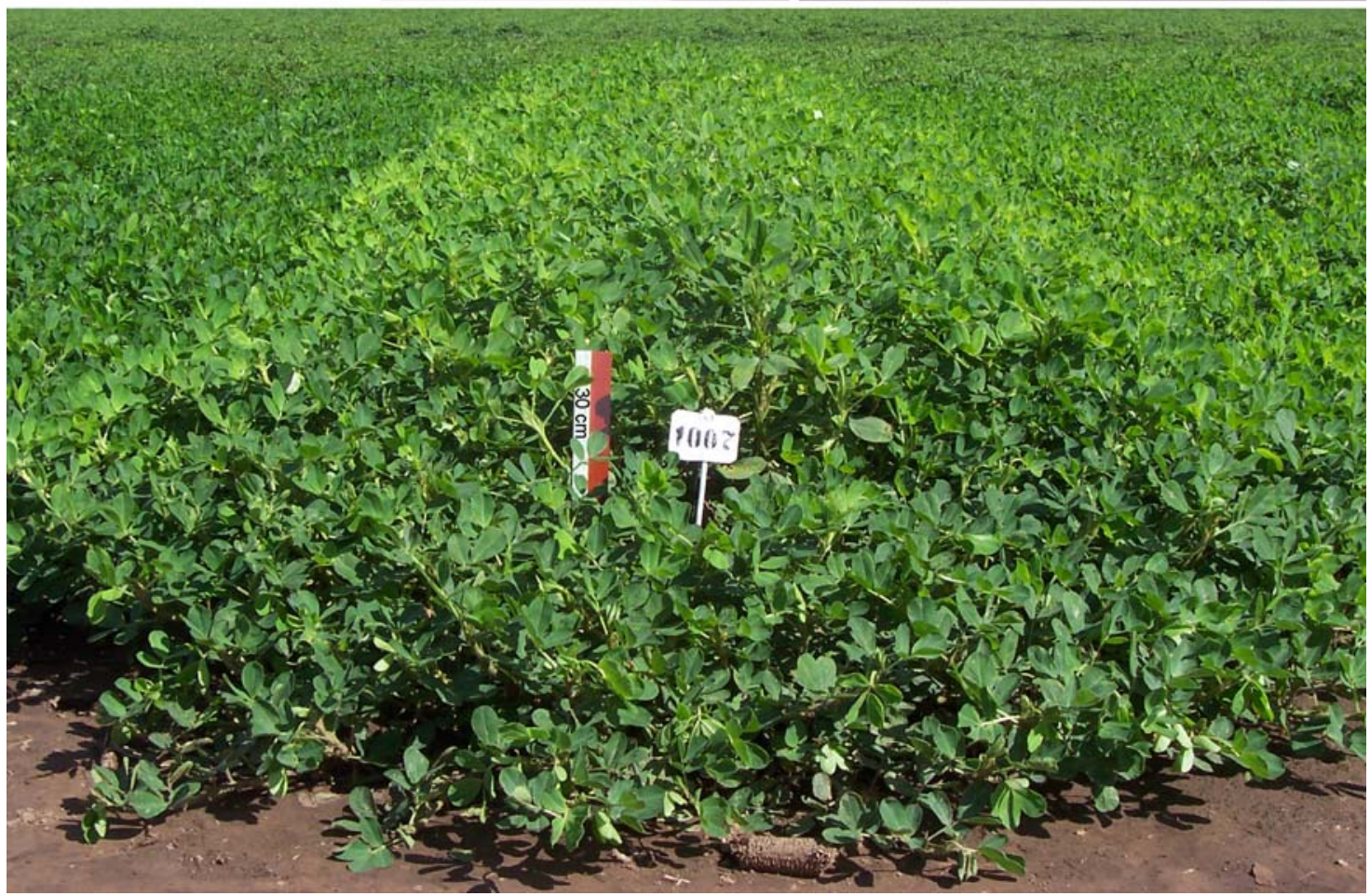




\section{Valluno rastrero}

Planta rastrera, grande, eje central bien aparente. Cerdas hasta el raquis.

Cajas: con retículo marcado, con joroba, sin pico. Granos colorado clarete.

$\begin{array}{rrlll}\text { 06/07 1008 } & 85 / 861760 & \text { US } 56 & \text { BPZ } & \begin{array}{l}\text { Santa Cruz,Valle Grande } \\ \text { Cochabamba, Cochabamba }\end{array}\end{array}$
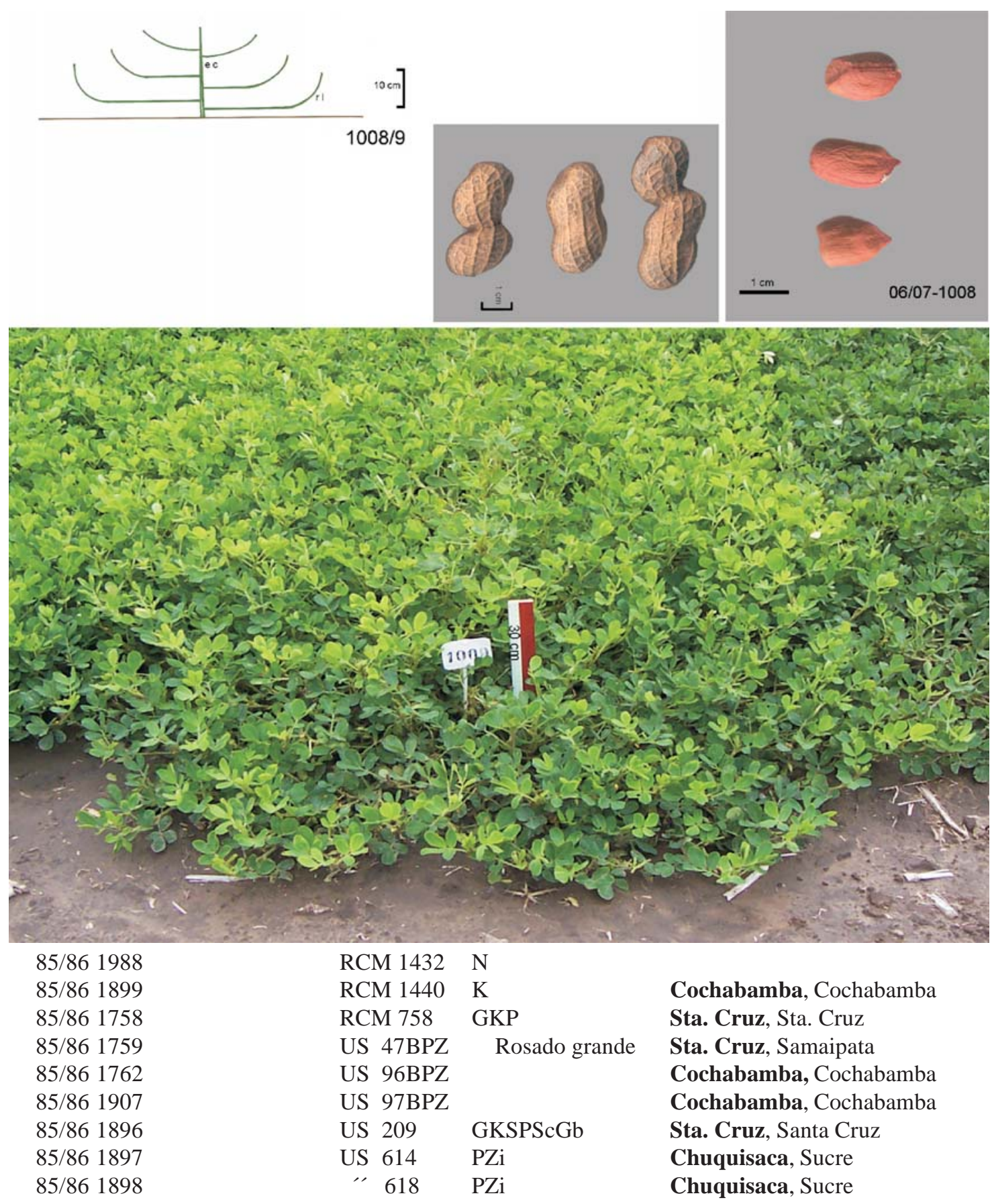


\section{Rastrero colorado de dos granos}

Planta rastrera, abierta; eje central aparente, con ramas que dan una forma piramidal a la planta. Cerdas hasta e el raquis. Verde oscuro. Muy atacado por viruela.

Cajas similares a Sara Maní con pico. Granos colorado fuerte.
06/07 1010
$85 / 861717$
US 29 GKBSPSc
Tarija,Villa Montes
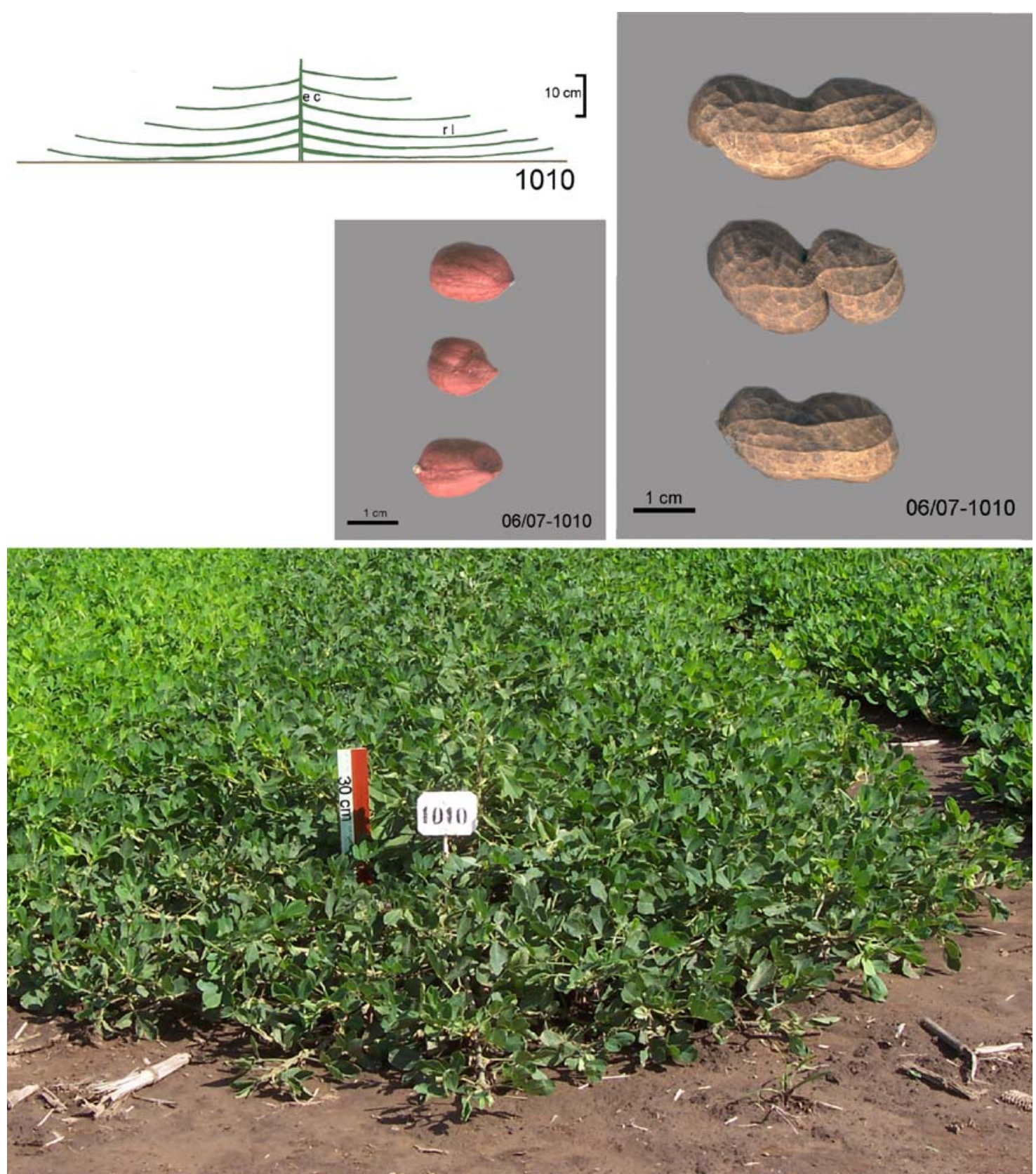


\section{Crema}

Planta rastrera, eje central no aparente. Estandarte sin aro. Cajas redondeadas, retículo grueso, sin carena, sin pico, 2 (3) granos salmón claro.

$$
\begin{array}{r}
\text { 06/07 } 1018 \\
1019
\end{array}
$$
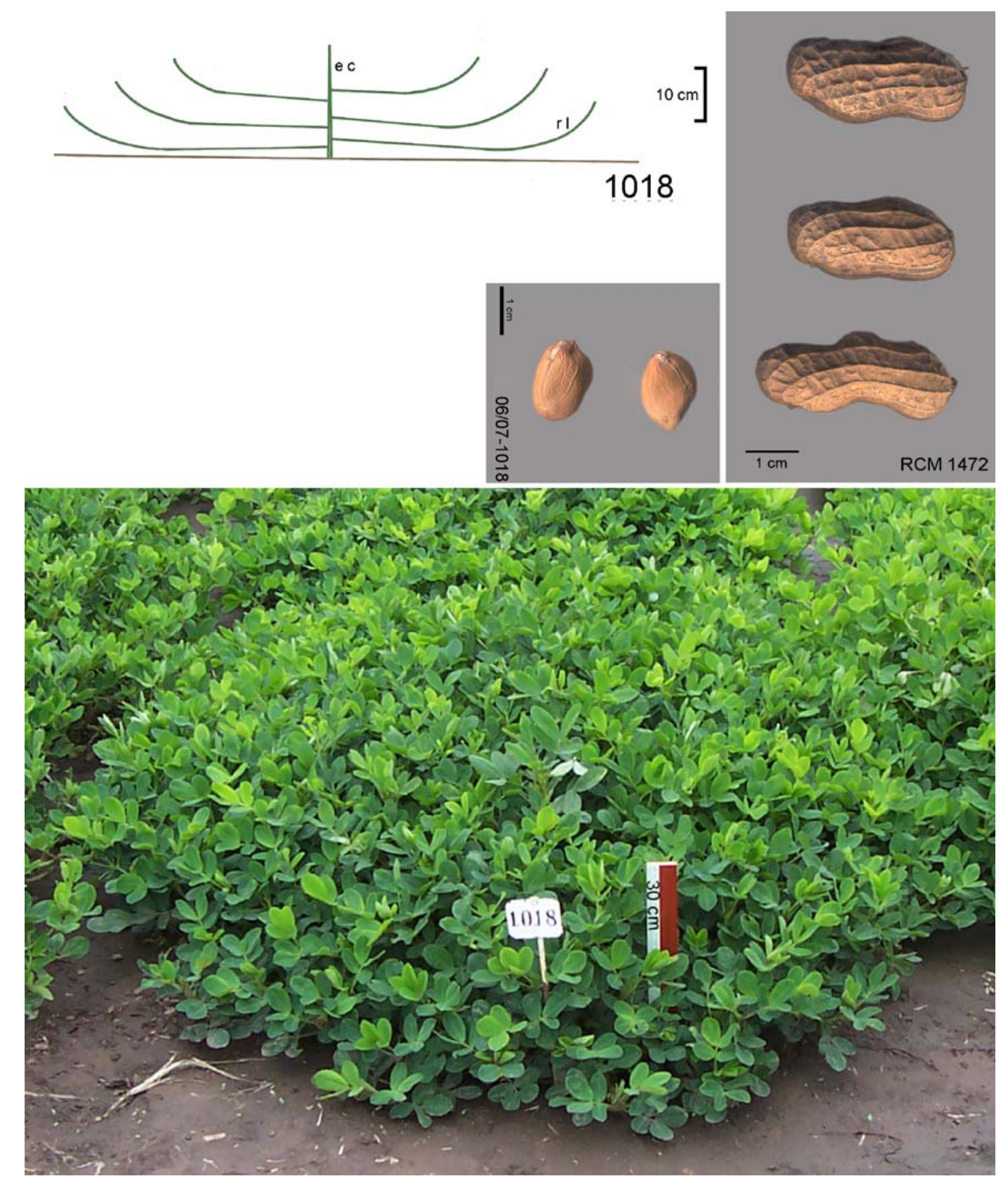

Tarija, San Telmo

Pando, Cobija 
Krapovickas, A.\& al., Las razas de maní de Bolivia

\begin{tabular}{|c|c|c|c|c|c|}
\hline 85/86 1727 & \multicolumn{2}{|c|}{ RCM 1474} & \multicolumn{2}{|l|}{ KFMo } & Tarija, Tarija \\
\hline 85/86 1729 & US & 8 & \multicolumn{2}{|l|}{ GKBSPSc } & Tarija, Bermejo \\
\hline 85/86 1730 & & 10 & & & \\
\hline 85/86 1731 & " & 103 & \multicolumn{2}{|l|}{$\mathrm{BPZ}$} & La Paz, La Paz \\
\hline 85/86 1733 & " & $310-2$ & \multicolumn{2}{|l|}{ KSBScCo } & Potosi ,Villazón \\
\hline 85/86 1734 & " & 311 & " & & " " " \\
\hline 85/86 1735 & " & 312 & \multicolumn{2}{|l|}{ ", } & " \\
\hline 85/86 1736 & " & 314 & \multicolumn{2}{|l|}{ " } & " \\
\hline 85/86 1737 & " & 319 & \multicolumn{2}{|l|}{ ", } & " \\
\hline 85/86 1738 & "' & 321 & \multicolumn{2}{|l|}{ " } & " \\
\hline 85/86 1739 & "' & 351 & \multicolumn{2}{|l|}{ BZCo } & La Paz, La Paz \\
\hline $85 / 861740$ & " & 355 & \multirow{2}{*}{ "' } & Crema & " \\
\hline 85/86 1741 & " & 360 & & ", & ", \\
\hline 85/86 1742 & "' & 361 & " & ", & $"$ \\
\hline 85/86 1743 & ", & 363 & ", & " & " \\
\hline 85/86 1744 & "' & 366 & " & " & $"$ \\
\hline 85/86 1745 & " & 367 & ", & " & " \\
\hline 85/86 1746 & " & 368 & ", & " & " \\
\hline 85/86 1747 & " & 369 & ", & " & " \\
\hline 85/86 1748 & " & 370 & ", & " & " \\
\hline 85/86 1749 & " & 374 & " & ", & " \\
\hline 85/86 1750 & " & 376 & " & Colorado & " \\
\hline 85/86 1751 & " & 378 & "' & Crema & " \\
\hline 85/86 1752 & " & 386 & BZCoJk & & La Paz, San Pedro (Coroico) \\
\hline 85/86 1753 & $"$ & 391 & " & Crema & La Paz, Caranavi \\
\hline 85/86 1754 & "' & 392 & " & Moro blanco & \\
\hline 85/86 1755 & $"$ & 805 & KSSc & & Tarija, Bermejo \\
\hline & " & 741 & Wi & & Beni, Rurrenabaque \\
\hline & " & 825 & Wi & & Pando, Cobija \\
\hline & " & 931 & "' & & Pando, Cobija \\
\hline & " & 1148 & $"$ & & Beni, Rurrenabaque \\
\hline
\end{tabular}




\section{Barcino}

Planta rastrera, eje central aparente, ramas rectas. Brote violáceo. Pocas cerdas en las estípulas (2-4).

Cajas redondas, algo estranguladas, sin carena ni pico; retículo grueso. Cajas con 2 granos negros.

06/07 1011

705 Wi Barcino

Beni, San Ignacio Moxos
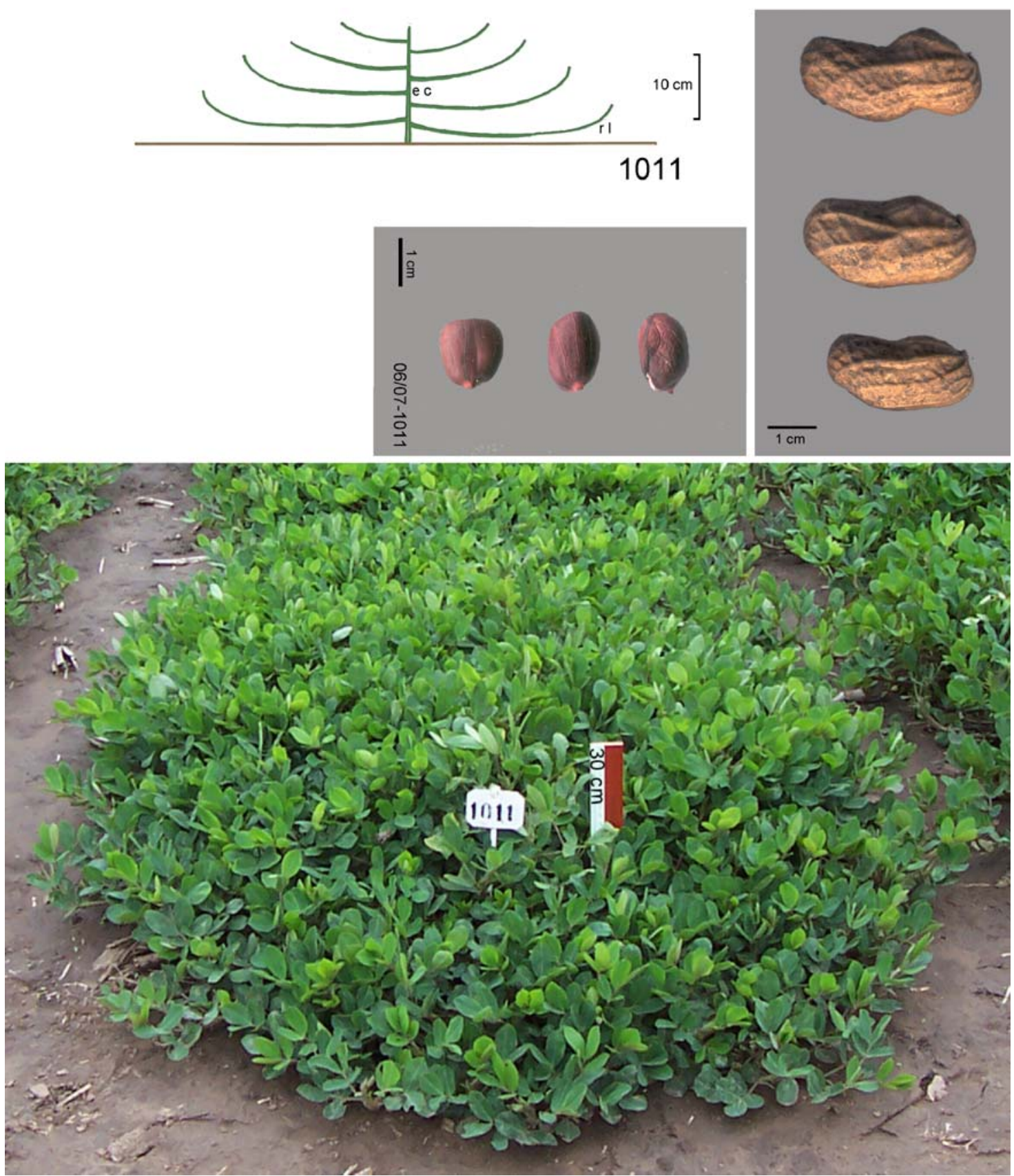


\section{Rastrero violáceo punta clara}

Planta rastrera, ramas rectas. Eje central aparente. Brote violáceo. Corola con las alas con manchas moradas.

Cajas g randes retículos marcado con pico y carena, (2)-3 granos violáceos, punta clara.

\section{6/07 1012 85/86 1994-3 US 345-2 KSBScCo～Sta. Cruz, Sta Cruz}
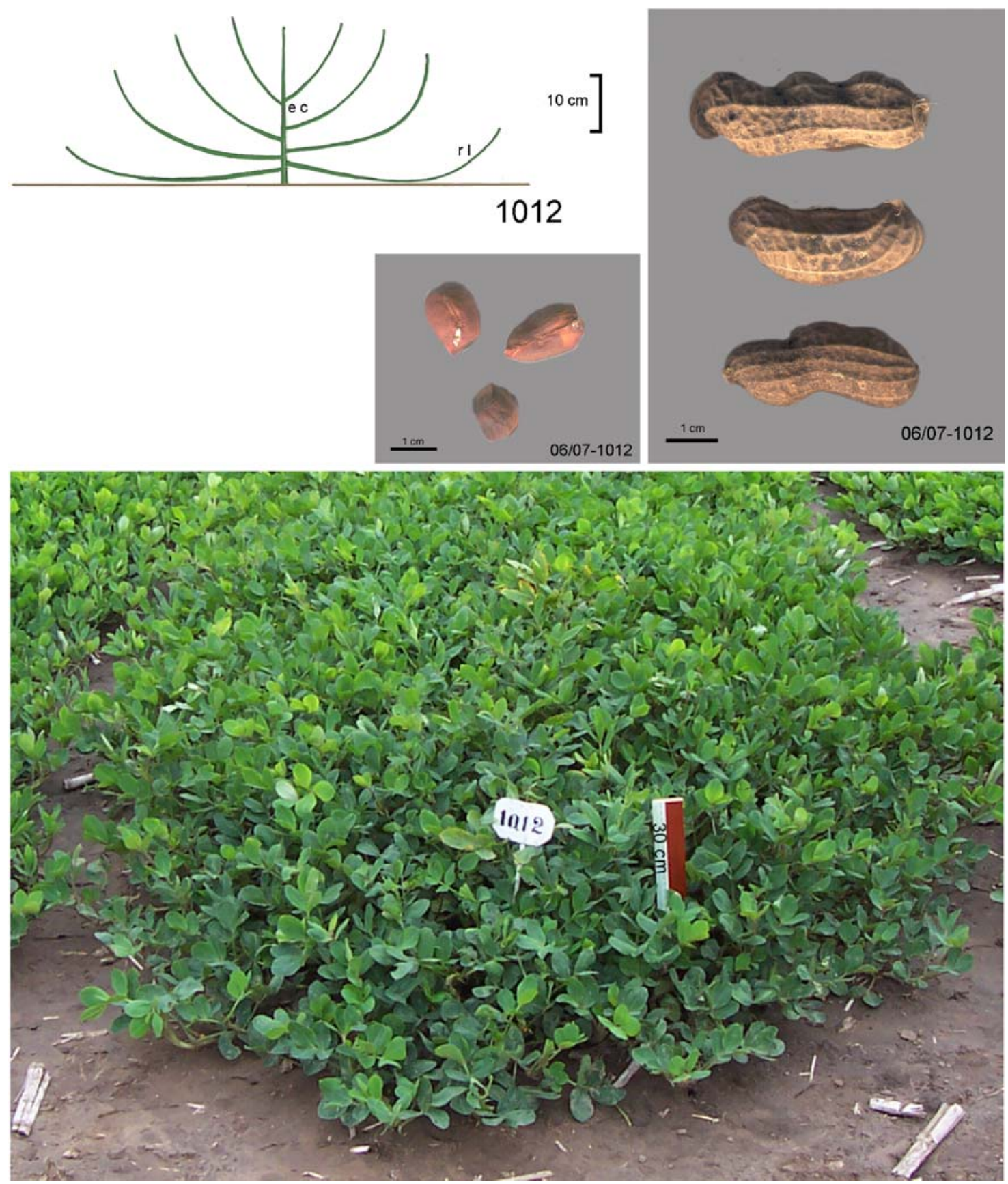


\section{Chaucha rosado}

Planta rastrera, eje central bien aparente,sin flores, Ramas sinuosas, con muchas reproductivas seguidas. Suceptible a viruela. Precoz ?

Cajas muy grandes, retículo marcados, venas longitudinales muy notorias, con constricción joroba y pico, 3- (2) granos rosados.

06/07 $1020 \quad 85 / 861989$
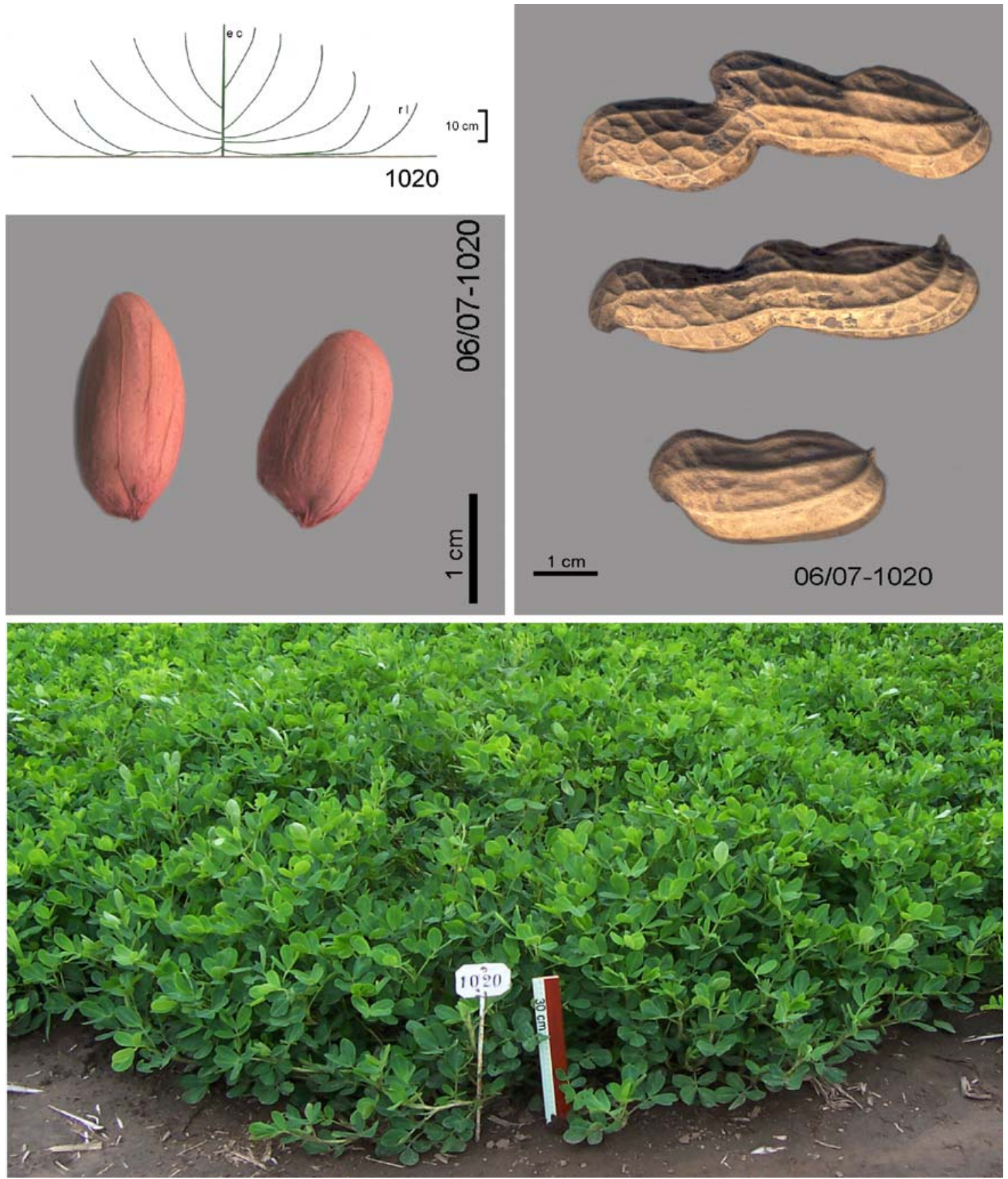

Sucre, Villa Serrano

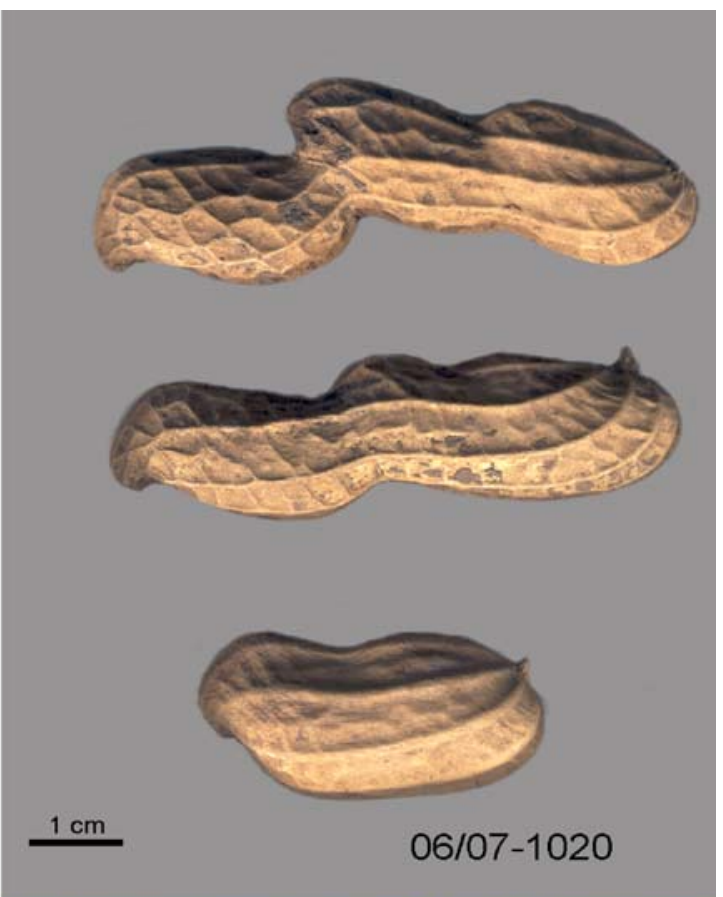




\section{Mani pico loro}

Planta rastrera, grande. Eje central muy aparente. Cerdas hasta en el raquis. Ramas sinuosas. Cajas muy grandes, hasta 4 granos. Colorado clarete.

$\begin{array}{rlll}\text { 06/07 } 1021 & 85 / 861704 & \text { US 99 BPZ } & \text { Cochabamba, Cochabamba } \\ 1022 & 85 / 861764 & \text { RCM 759 GKP } & \text { Sta. Cruz, Santa Cruz }\end{array}$
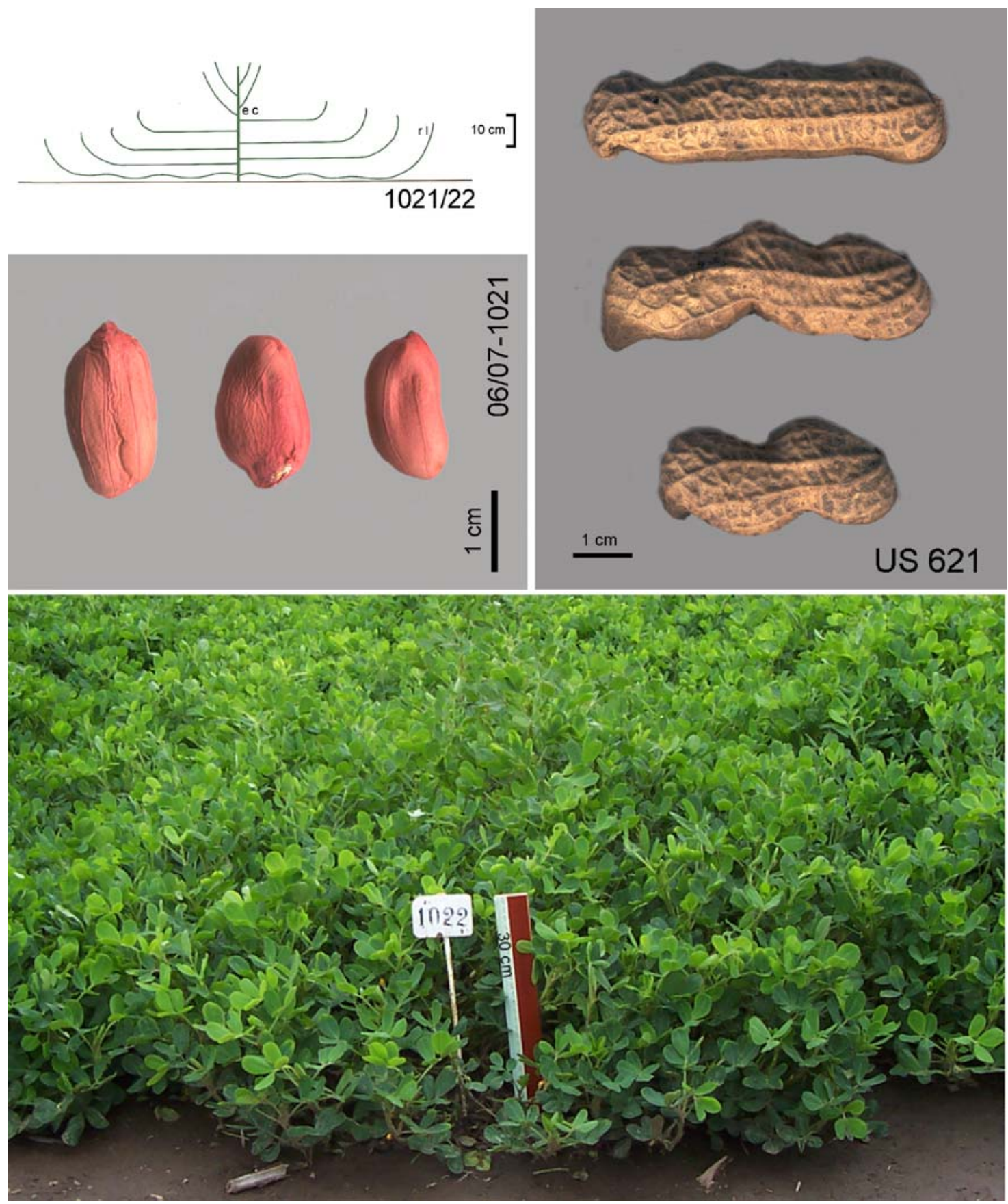


\begin{tabular}{|c|c|c|c|}
\hline 85/86 1766 & US 47 & Rosado grand & e Sta. Cruz, Samaipata \\
\hline 85/86 1767 & " 50 & " & Sta. Cruz, Puente de Mataral \\
\hline 85/86 1768 & " 55 & " & Sta. Cruz Valle Grande \\
\hline 85/86 1770 & " 56 & " & \\
\hline 85/86 1771 & ' 58 & " & \\
\hline 85/86 1970 & " 59 & " & \\
\hline 85/86 1776 & $\because 77$ & Rosado fuerte & Sta. Cruz, S. Isidro \\
\hline 85/86 1779 & " 79 & " & " \\
\hline 85/86 1781 & "' 80 & " & \\
\hline 85/86 1783 & " 81 & " & Cochabamba, Teneria- Aiquile \\
\hline 85/86 1784 & ' 87 & & Cochabamba, Pogo-Mizque \\
\hline 85/86 1787 & " 88-1 & " & ", Mizque \\
\hline 85/86 1795 & " 89 & " & " \\
\hline 85/86 1788 & " 90 & " & " \\
\hline 85/86 1790 & " 91 & " & ", \\
\hline 85/86 1792 & ” 92 & " & " $\quad$ " \\
\hline 85/86 1794 & " 94 & Cdo. Grande & Cochabamba-Cochabamba \\
\hline 85/86 1796 & " 96 & " & "r $\quad$ " \\
\hline 85/86 1864-2 & " 349 & KSBScCo & Sta. Cruz, Santa Cruz \\
\hline 85/86 1802 & "609-1 & $\mathrm{PZi}$ & Chuquisaca, Sucre \\
\hline 85/86 1804 & " 610 & " & " \\
\hline 85/86 1806 & " 611 & " & " \\
\hline 85/86 1807 & " 612 & " & " $\quad$ " \\
\hline 85/86 1825 & " 614 & " & Chuquisaca, Sucre \\
\hline 85/86 1822 & " 615 & " & Chuquisaca, Sucre \\
\hline 85/86 1810 & ” 617 & " & "r \\
\hline 85/86 1812 & " 618-1 & " & ", \\
\hline 85/86 1813 & " 621 & " & Chuquisaca, Padilla \\
\hline $85 / 861816-3$ & ” 622 & " & Chuquisaca, La Calera, Padilla \\
\hline 85/86 1817 & " 623-1 & Larguillo & Chuquisaca, Villa Serrano \\
\hline 85/86 1819 & ” 624 & Pico loro & ") \\
\hline 85/86 1820 & " 625 & ", & " \\
\hline 85/86 1821 & " 626 & " & " \\
\hline 85/86 1826 & " 628-1 & " & Chuquisaca, Villa Serrano \\
\hline 85/86 1830 & ' 629-1 & " & $\begin{array}{lll}\prime & \prime & \end{array}$ \\
\hline 85/86 1832 & " 630 & Larguillo & $\begin{array}{lll}\prime & \prime & \prime\end{array}$ \\
\hline 85/86 1834 & "631-1 & " & Chuquisaca, Villa Serrano \\
\hline 85/86 1846 & ” 632 & " & ") \\
\hline 85/86 1847 & " 633-1 & " & ', \\
\hline 85/86 1837 & "634-1 & " & Chuquisaca, Pozos \\
\hline 85/86 1841 & " 637 & & \\
\hline 85/86 1984 & "' 638 & & ', Villa Serano \\
\hline 85/86 1843 & " 836-1 & $\mathrm{KSScCr}$ & Tarija, Entre Ríos \\
\hline
\end{tabular}




\section{Yungueño}

Planta rastrera, grande. Eje central muy aparente. Cerdas hasta el raquis.

Cajas con dos a tres granos, reticulo grueso, con algo de pico, granos colorados.

\begin{tabular}{|c|c|c|c|c|}
\hline 06/07 1025 & 85/86 1909 & US 107 & BPZ & La Paz, La Paz \\
\hline 1026 & 85/86 1910 & US 380 & BZCoJk & La Paz, S. Pablo (Coroico) \\
\hline
\end{tabular}
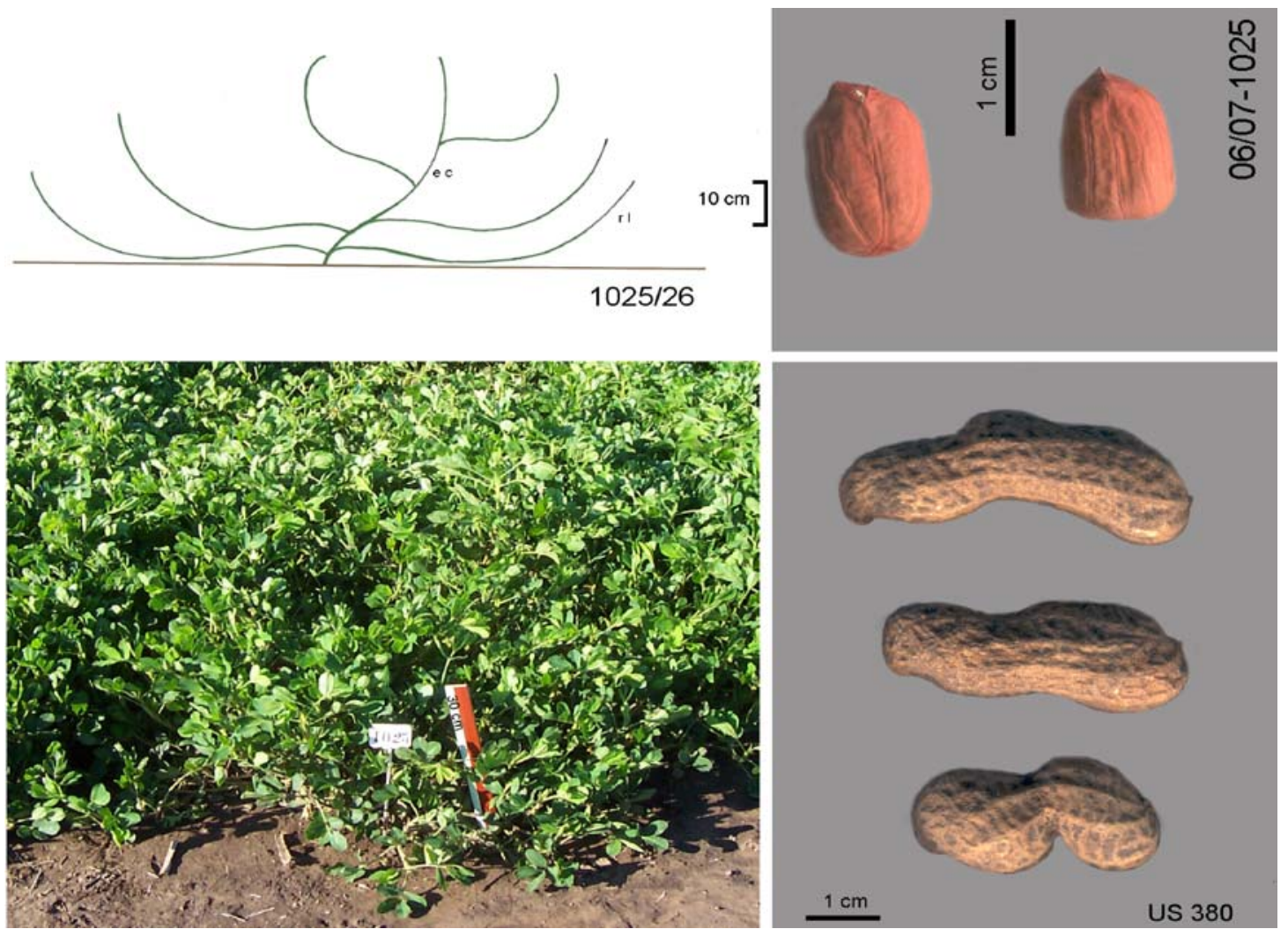

85/86 1908

$85 / 861911$

85/86 1912

85/86 1913

85/86 1915

85/86 1918

85/86 1921

85/86 1922

85/86 1923

85/86 1926

85/86 1927

85/86 1929

85/86 1930

85/86 1935

85/86 1936

85/86 1937

85/86 1939

\begin{tabular}{|c|c|c|}
\hline US 106 & BPZ & \\
\hline ' 381 & BZCoJk & Criollo \\
\hline “ 382 & m & "' \\
\hline 385 & ", & Colorad \\
\hline $102-A$ & BPZ & Yungueî \\
\hline 104 & "' & \\
\hline 105 & " & Criollo \\
\hline 106 & "' & \\
\hline 107 & "' & \\
\hline 108 & $"$ & \\
\hline " 109 & " & \\
\hline " 110 & " & \\
\hline 111 & " & \\
\hline " 112 & " & \\
\hline " 113 & " & \\
\hline " 114 & m & \\
\hline 379 & BZCoJk & \\
\hline
\end{tabular}

La Paz., La Paz

La Paz, Yalaca (Coroico)

La Paz, Munaipata

La Paz, Coroico

La Paz, La Paz

La Paz, Chulumani (S.Yungas)

a Paz, Sanani

La Paz, Chulumani (S. Yungas)

La Paz, Rancho Chico de Irupana

La Paz, Capani-Irupana

La Paz, Irupana

La Paz, Chulumani

La Paz, B. Huaylluco

La Paz, Sacawaya-S. Yungas

La Paz, Sirupaya

La Paz, S. Pedro (Coroico) 


\section{Ronco}

Planta en cojín compacto, ramas rectas, cubre los surcos. EC no aparente a aparente. Cajas muy grandes retículo grueso, con 2-3 ganos salmón.
$06 / 07$
$1023 \quad 85 / 861811$
US 618
1024
Chuquisaca, Sucre-Monteagudo
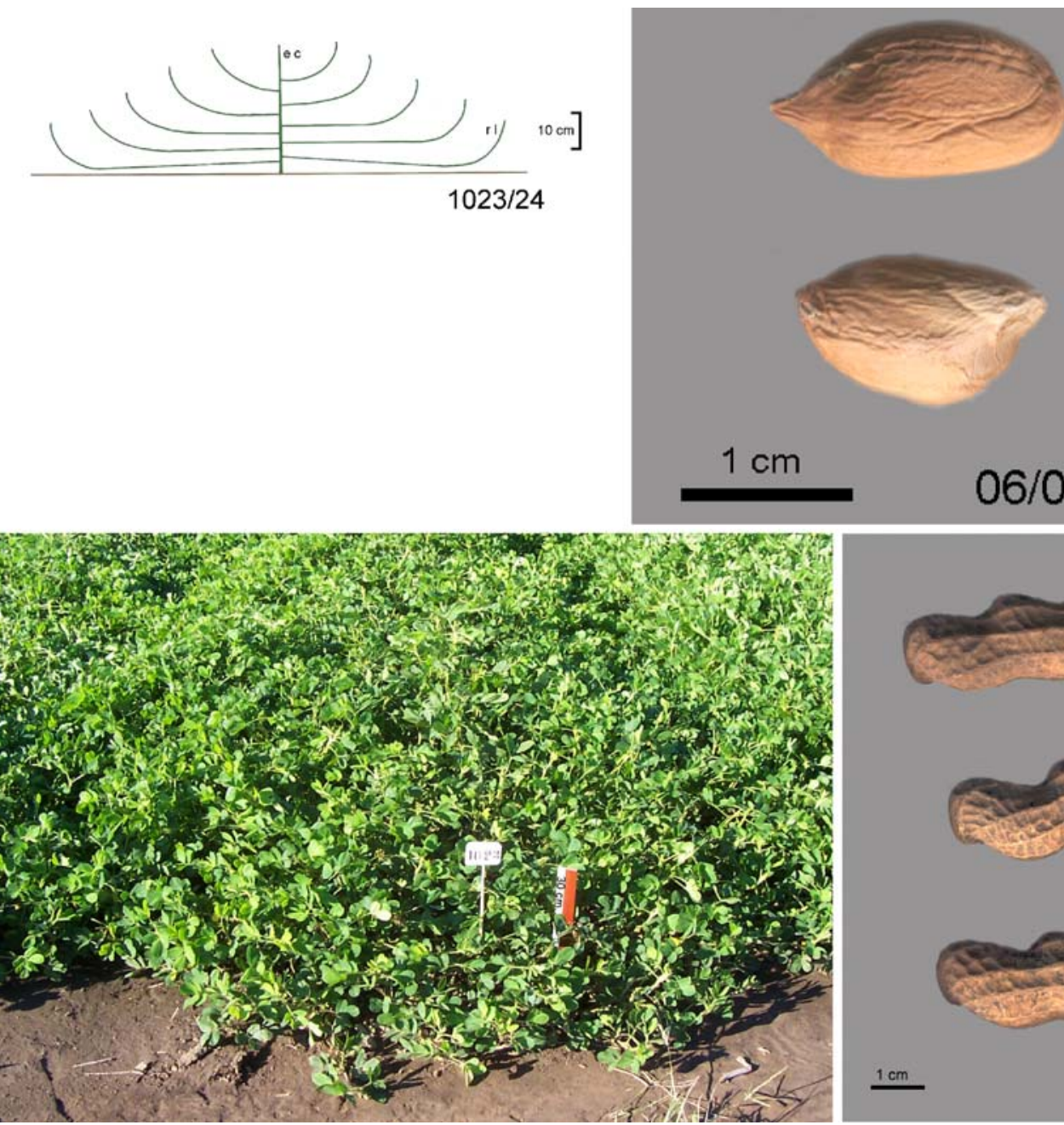

85/86 1857

85/86 1801

85/86 1818

85/86 1827

85/86 1833

85/86 1835

85/86 1836

85/86 1838

85/86 1844

85/86 1842

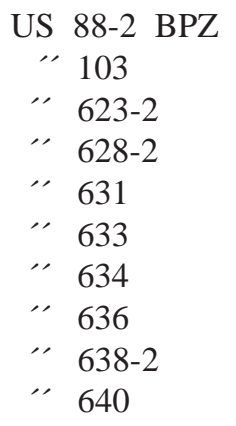

\section{6/07-1023}

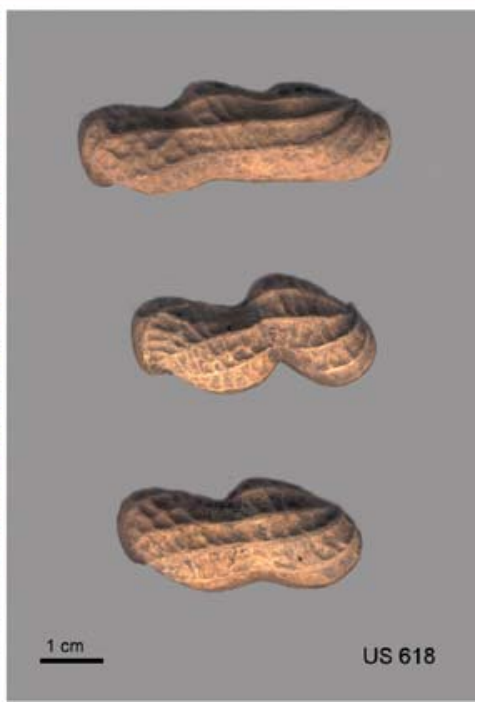

Cochabamba, Mizque

La Paz, La Paz

Chuquisaca, Villa Serrano

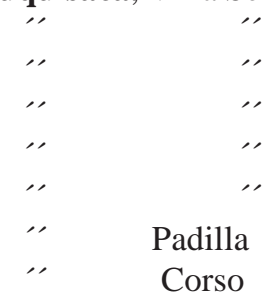




\section{Sopachuy salmón}

Planta semirastrera a cojín grande, muy ramificada. EC algo aparene.

Cajas generalmente con dos granos, raro 3, reticulo fino. Granos salmón.

$\begin{array}{rlll}\text { 06/07 } 1027 & 85 / 861942 & \text { RCM 1480 KMoF } & \text { Tarija, San Simóm } \\ 1028 & 85 / 861964 & \text { US 29 GKBSPSc } & \text { Tarija, Villa Montes }\end{array}$

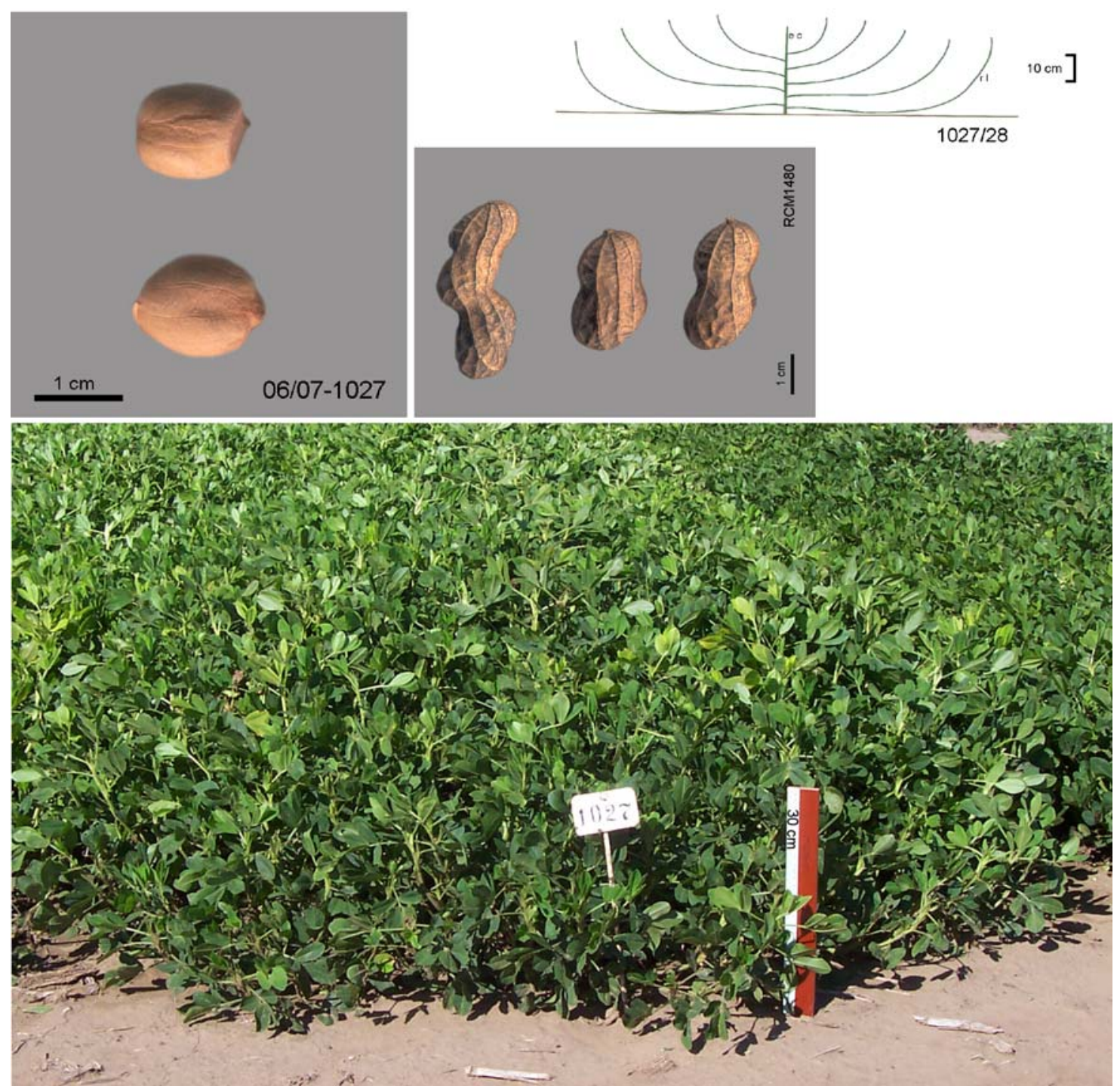

85/86 1944

85/86 1809

85/86 1950

85/86 1951

85/86 1952

85/86 1892

85/86 1953

\begin{tabular}{|c|c|}
\hline RCM & $1457 \mathrm{~N}$ \\
\hline US & $615 \mathrm{PZi}$ \\
\hline 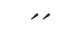 & $825 \mathrm{KSSc}$ \\
\hline & 827 ' \\
\hline & $832-1{ }^{\prime \prime}$ \\
\hline & -b" \\
\hline & $833-3$ \\
\hline
\end{tabular}

Chuquisaca, Sucre

Tarija, Entre Ríos

$\begin{array}{lll}\text {," } & \text {, } & \text {, } \\ \text {, } & \text {, } & \text {, } \\ \text {, } & \text {, } & \text {, } \\ \text {, } & \text {,. } & \text {, }\end{array}$




\section{Sopachuy colorado}

Granos colorado clarete.
06/07 1029
85/86 2014
RCM 1429 N
85/86 1941 RCM $1475 \mathrm{KMoF}$
Cochabamba, Mairana
1030
Tarija, Tarija
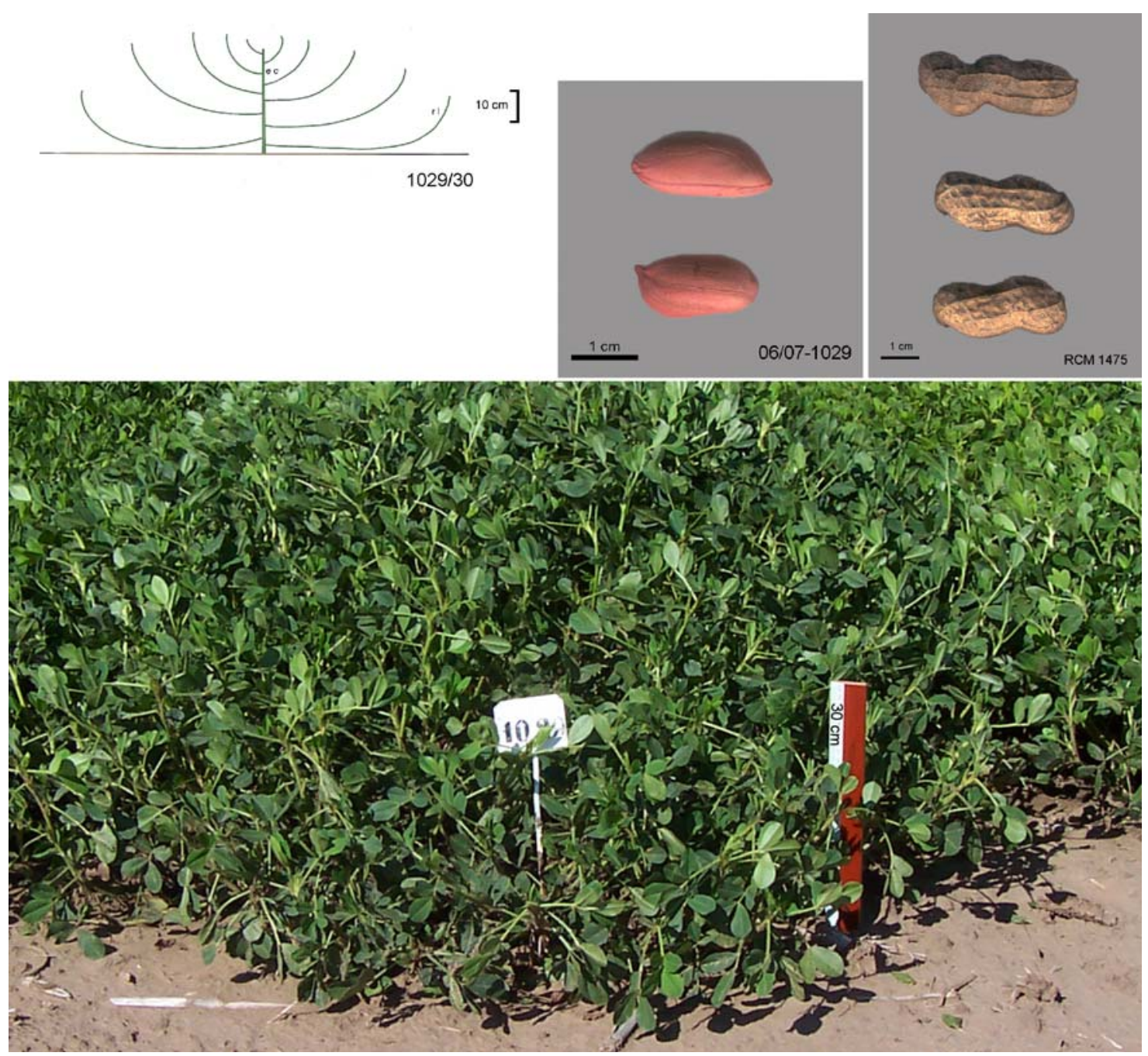

85/86 1963

85/86 1943

85/86 2039

85/86 1945

85/86 2042-1

85/86 1799

85/86 2023

85/86 1876

85/86 1879

85/86 1894

$$
\begin{aligned}
& \text { RCM } 1470 \mathrm{~K} \\
& \text { RCM } 1481 \text { KMoF } \\
& \text { US } 48 \text { BPZ } \\
& \text { " } 49 \text { "' } \\
& \text { " } 69 \text { " } \\
& \text { " } 97 \text { " } \\
& \text { " 814-2 KSSc } \\
& \text { ". 816-1 "' } \\
& \text { " 827-2 ", } \\
& \text { ". 828-4 " }
\end{aligned}
$$

Sta, Cruz, Samaipata

Tarija, Tarija

Sta. Cruz, Yerba Buena

$$
\begin{array}{lll}
\text {," } . & \text { Agua Clara } \\
& & \text { Samaipata }
\end{array}
$$

Cochabamba, Cochabamba

Tarija, Tarija

Tarija, Tarija

Tarija, Entre Ríos 


\section{San José de Chicaludo}

Planta cojín compacto, eje central no aparente; cerdas hasta en el raquis.

Cajas grandes, 2-3 granos colorado clarete.

06/07 1031 85/86 2102 RCM 1443 N La Paz, N.Yungas San Jose de Chicaludo
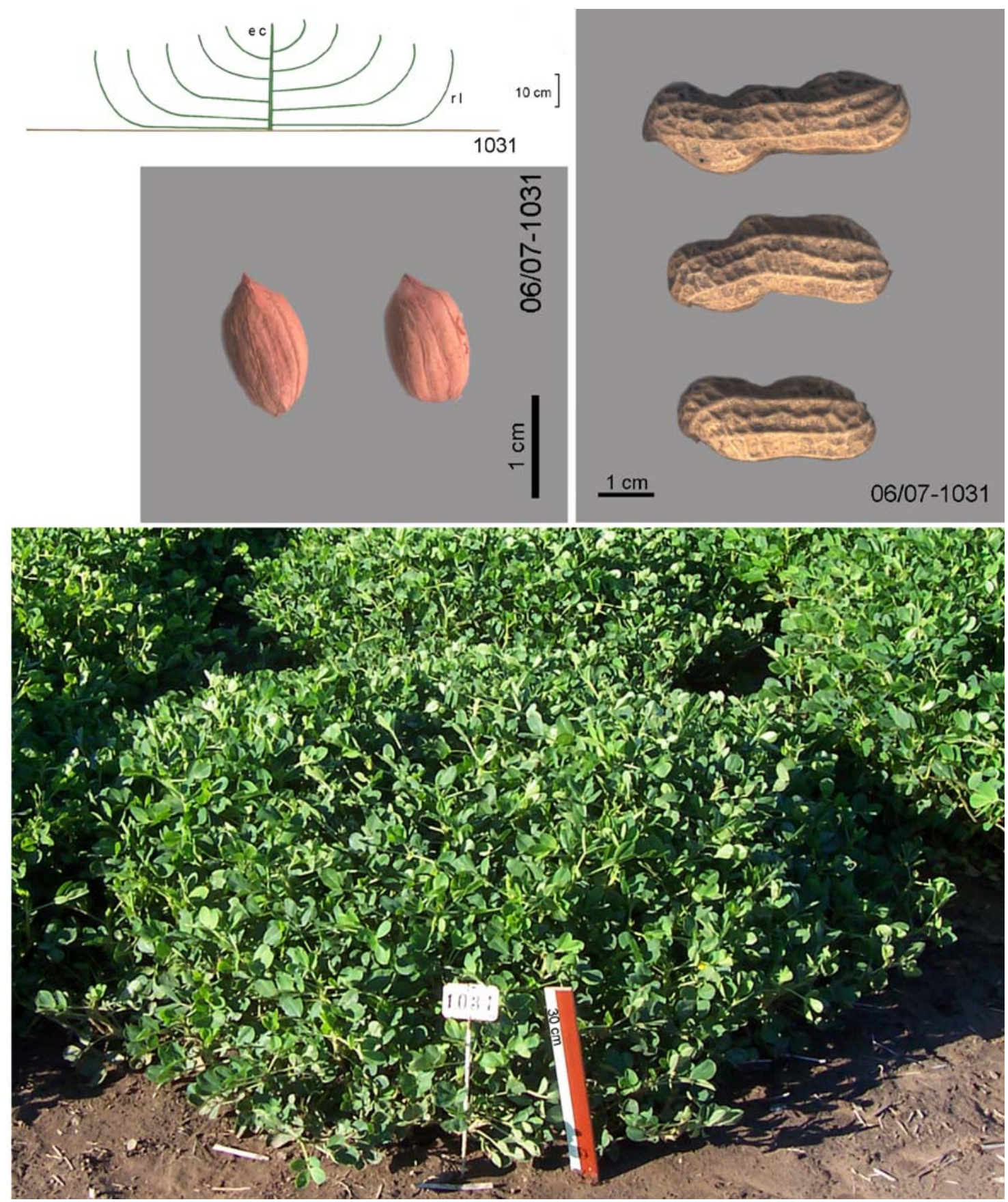


\section{Churco}

Planta cojín grande.

Cajas grandes, con 2-3 granos, color salmón.

06/07 1034 85/86 1856 US 89 BPZ Cochabamba, Mizque
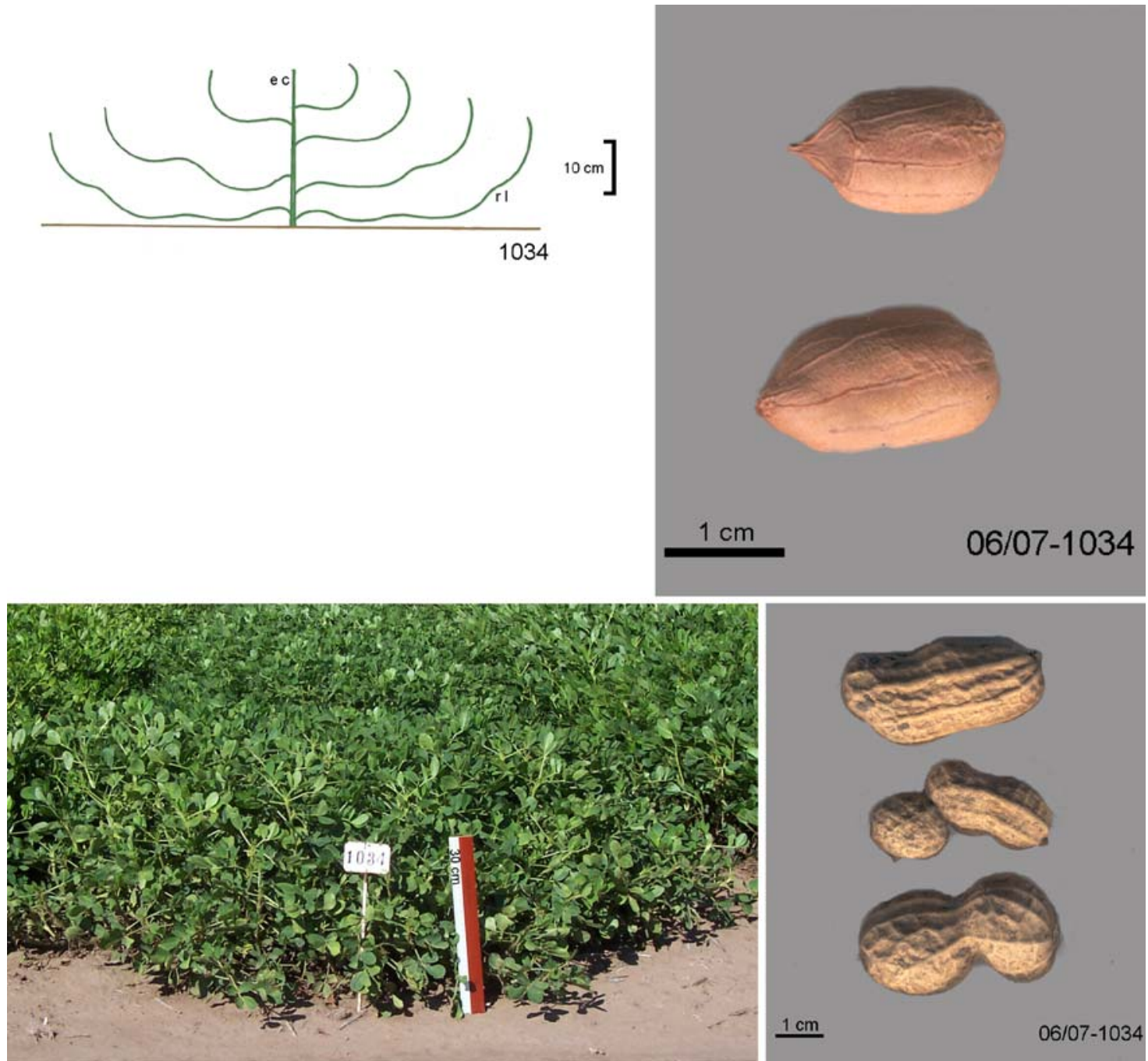

85/86 1854

85/86 1848

85/86 1849

85/86 1791

85/86 1861

85/86 1864-1

$85 / 86$ 1865-1

85/86 1883

85/86 1893

\begin{tabular}{|c|c|c|}
\hline RCM & 1429 & $\mathrm{~N}$ \\
\hline ", & 1483 & KMoF \\
\hline US & 11 & GKBSPSc \\
\hline 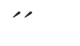 & 91 & ", \\
\hline & 92 & " \\
\hline 'ו & 349 & KSBScCo \\
\hline " & 616 & $\mathrm{PZi}$ \\
\hline ' & 806 & KSSc \\
\hline & 832-f & \\
\hline
\end{tabular}

Sta. Cruz, Mairana

Tarija, Tarija

Tarija, Bermejo<smiles>C1CCC1</smiles>

Sta. Cruz, Santa Cruz Chuquisaca, Sucre

Tarija, Tarija

Tarija, Entre Ríos 


\section{Churco de Tarija}

Planta cojín compacto; eje central no aparente. Cerdas hasta el raquis.

Cajas grandes tres granos algunos de 2 granos, retículo fino. Colorado clarete.
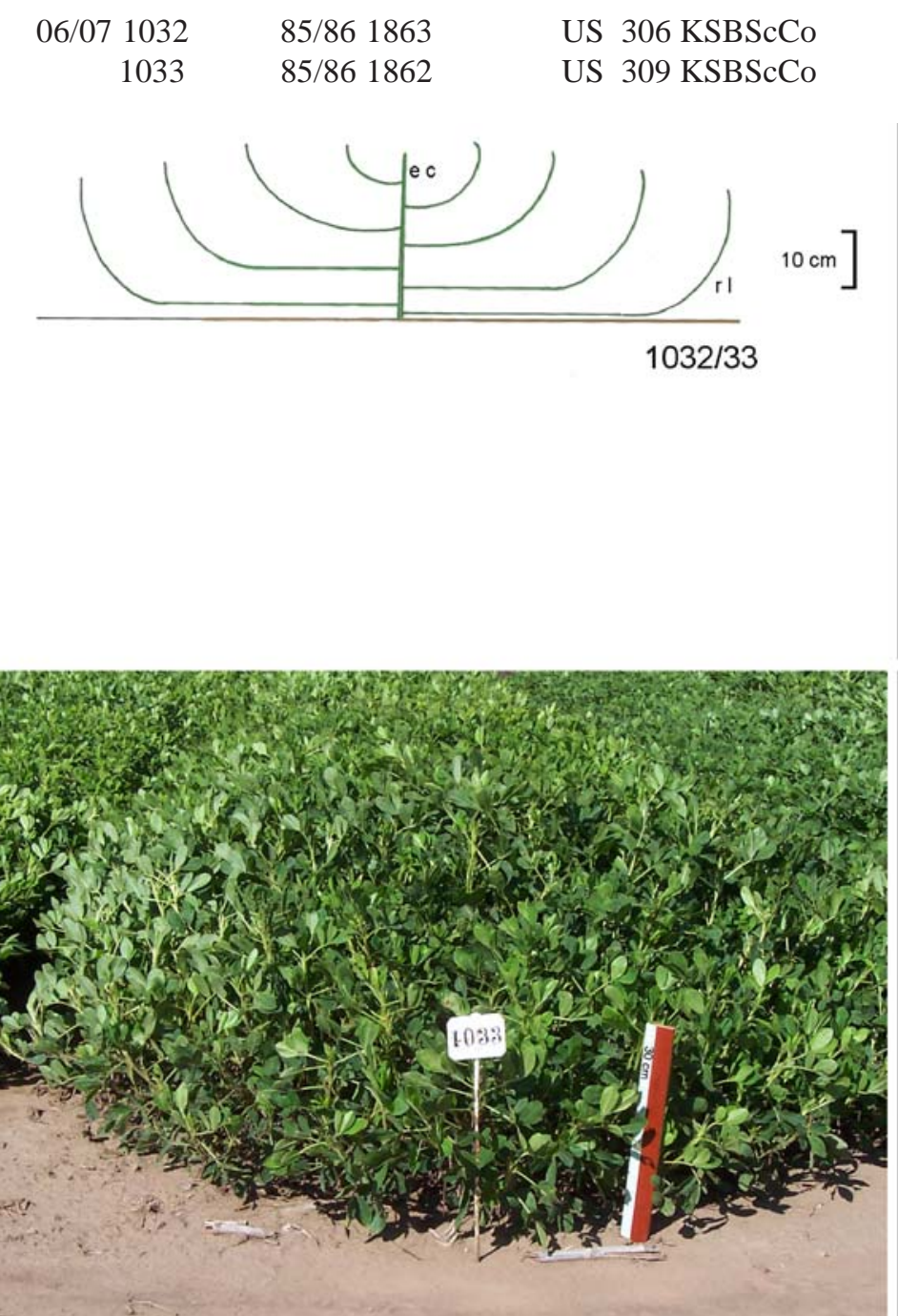

$85 / 862101$

$85 / 861851$

$85 / 861858$

85/86 1871

$85 / 861873$

$85 / 861874$

$85 / 861875$

$85 / 861889$

$85 / 861877$

85/86 1878

\begin{tabular}{|c|c|c|}
\hline RCM & 1462 & K \\
\hline US & 22 & GKSPSc \\
\hline & 90 & PBZ \\
\hline & $807-3$ & KSSc \\
\hline & $808-2$ & " \\
\hline & 809 & " \\
\hline , & $810-1$ & " \\
\hline " & 811-1 & " \\
\hline '" & 815 & " \\
\hline & 817 & $"$ \\
\hline & 821 & $"$ \\
\hline
\end{tabular}

Potosi, Villazón

Potosi, Villazón
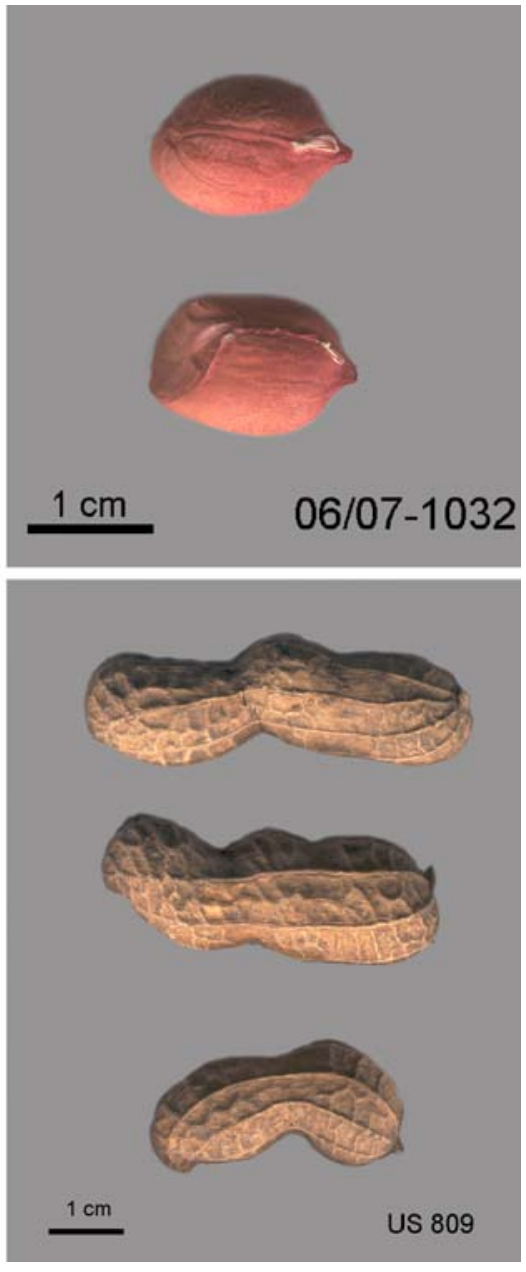

Cochabamba, Cochabamba

Tarija, Carapari

Cochabamba, Mizque

Tarija, Tarija

$\begin{array}{ll}\text { ". } & \text { ". } \\ \text { ". } & \text { ". } \\ \text { " } & \text { ". } \\ \text { ". } & \text { ". } \\ \text { " } & \text { ". } \\ \text { ". }\end{array}$




\section{Cojín Valluno}

Planta cojín compacto, ramas rectas; eje central no aparente. Sin cerdas.

Cajas con 2 granos; retículo más o menos diluído . Granos colorado fuerte.
06/07 1038
85/86 1985
US 342 KSBScCo
Sta. Cruz, Santa Cruz 1039
85/86 1900
Sta. Cruz, Santa Cruz
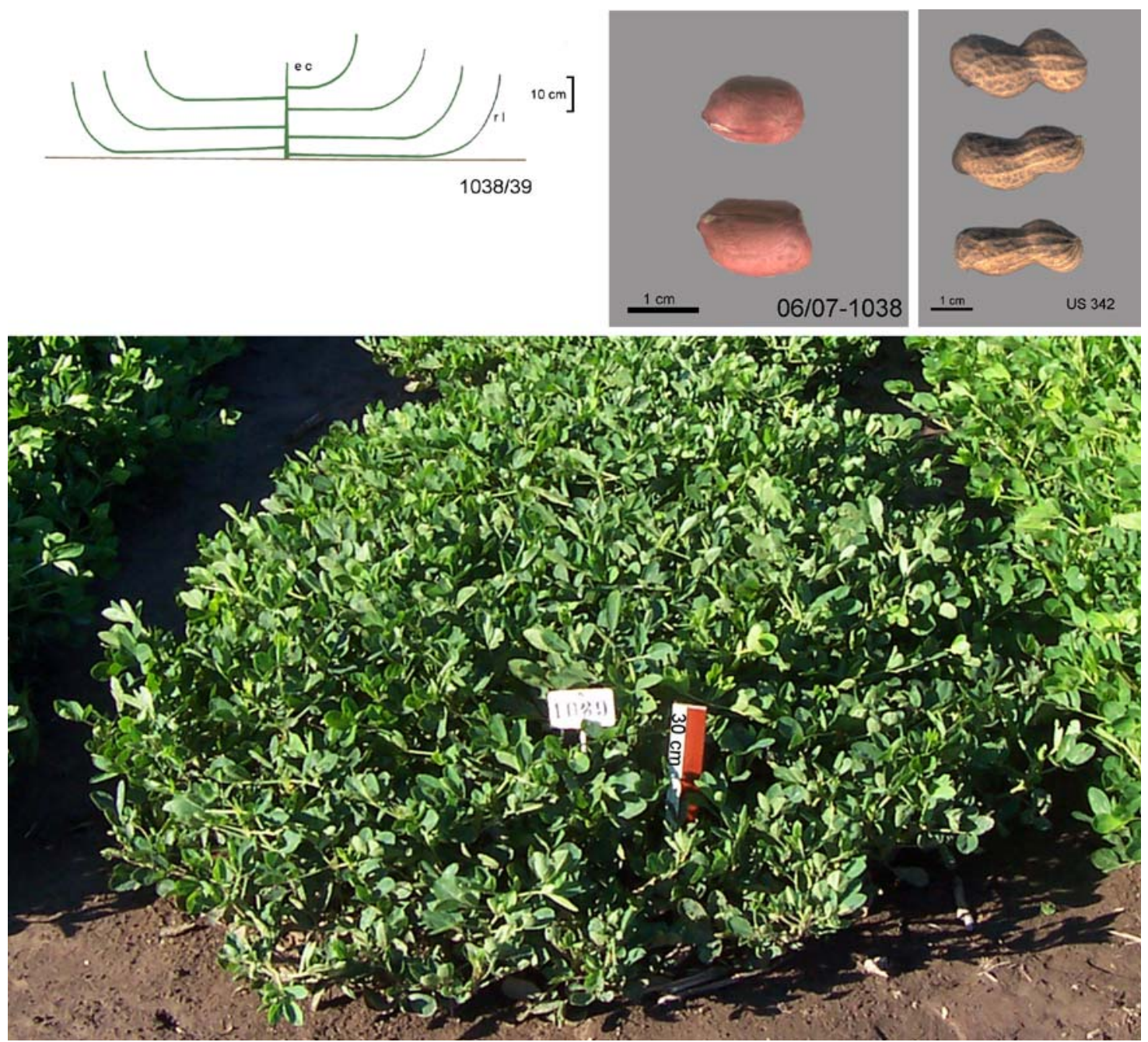

85/86 2098

85/86 2107

85/86 2037

85/86 1757

$85 / 862100$

85/86 2108

85/86 1901

85/86 1902

85/86 1903

\begin{tabular}{|c|c|c|}
\hline RCM & 1434 & $\mathrm{~N}$ \\
\hline & 1450 & $\mathrm{~N}$ \\
\hline & 1451 & $\mathrm{~N}$ \\
\hline & 1453 & $\mathrm{~K}$ \\
\hline & 1454 & $\mathrm{~K}$ \\
\hline & 1469 & $\mathrm{~K}$ \\
\hline US & $330-2$ & KSBScCo \\
\hline 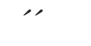 & 343-2 & "' \\
\hline " & 344 & " \\
\hline
\end{tabular}

Sta. Cruz, Mairana

Cochabamba, Cochabamba

Sta. Cruz, Samaipata

Sta. Cruz, Santa Cruz

Sta. Cruz, Santa Cruz

Sta. Cruz, Santa Cruz 


\section{Sara maní}

Planta cojín grande, ramas muy sinuosas; eje central poco aparente, n1, n2 ,n3 y n4. Con cerdas.

Cajas con constricción, pico y carena, retículo marcado, notorio. 2 -(3) granos salmón.

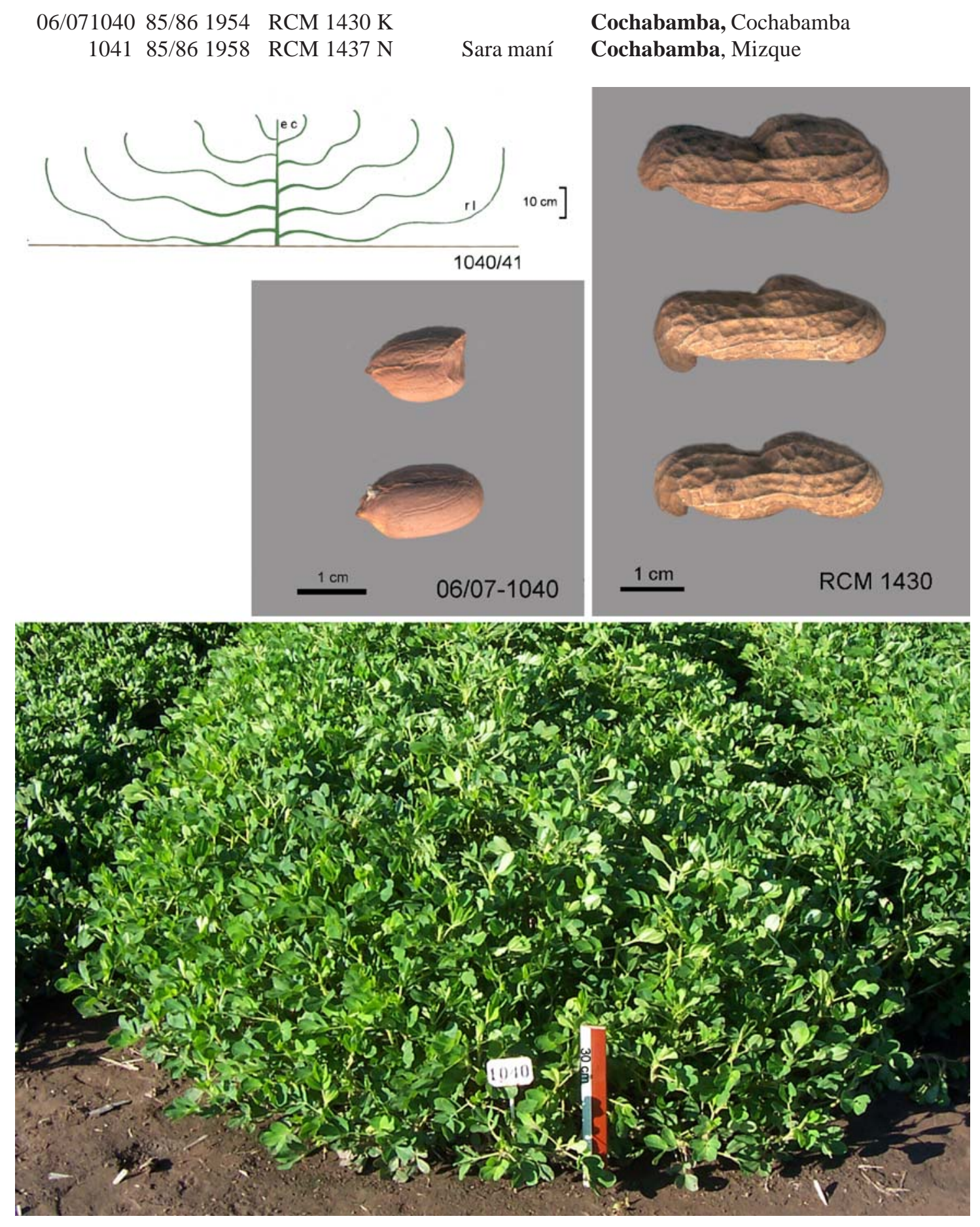

Cochabamba, Cochabamba

Cochabamba, Mizque 
BONPLANDIA 18(2). 2009

$\begin{array}{ll}85 / 86 & 1955 \\ 85 / 86 & 1956 \\ 85 / 86 & 1957 \\ 85 / 86 & 1959 \\ 85 / 86 & 1961 \\ 85 / 86 & 1962 \\ 85 / 86 & 1965 \\ 85 / 86 & 1966 \\ 85 / 86 & 1967 \\ 85 / 86 & 1968 \\ 85 / 86 & 1969 \\ 85 / 86 & 1971 \\ 85 / 86 & 1972 \\ 85 / 86 & 1973 \\ 85 / 86 & 1975 \\ 85 / 86 & 1977 \\ 85 / 86 & 1978 \\ 85 / 86 & 1979 \\ 85 / 86 & 1981 \\ 85 / 86 & 1983\end{array}$

\begin{tabular}{|c|c|c|c|}
\hline RCM & 1431 & & \\
\hline "' & 1433 & & \\
\hline , & 1436 & & \\
\hline " & 1438 & & Sara maní \\
\hline " & 1441 & & \\
\hline " & 1468 & K & \\
\hline US & 50 & BPZ & \\
\hline "' & 52 & "' & \\
\hline "' & 54 & " & \\
\hline " & 58 & ', & \\
\hline m & 59 & $"$ & \\
\hline "' & 62 & "' & \\
\hline "r & 63 & ", & \\
\hline " & 64 & " & \\
\hline "' & 67 & "' & \\
\hline "' & 82 & ' & \\
\hline "r & 83 & ", & \\
\hline " & 84 & " & \\
\hline " & 85 & "' & \\
\hline " & $86-$ & "' & \\
\hline
\end{tabular}

Cochabamba, Cochabamba

Cochabamba, Cochabamba Sta. Cruz, Samaipata

Sta. Cruz, Puente de Mataral

Sta. Cruz, Lagunilla

Sta. Cruz, Vallegrande

Sta Cruz, Vallegrande

Sta. Cruz, Trigal

Sta. Cruz, Cochabambita

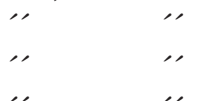

Cochabamba, Tenería Aiquile

Cochabamba, Mesa Rancho ,.

Cochabamba, Rumicancha 


\section{Negro cojín}

Planta en cojín compacto, casi rastrero. Eje central no aparente, ramas erectas. Brote violáceo. Cerdas hasta el raquis.

Cajas con pico marcado, 3 granos, negros.
06/07 1042
85/86 1994-2
US 345-2 KSBScCo
Sta. Cruz, Santa Cruz
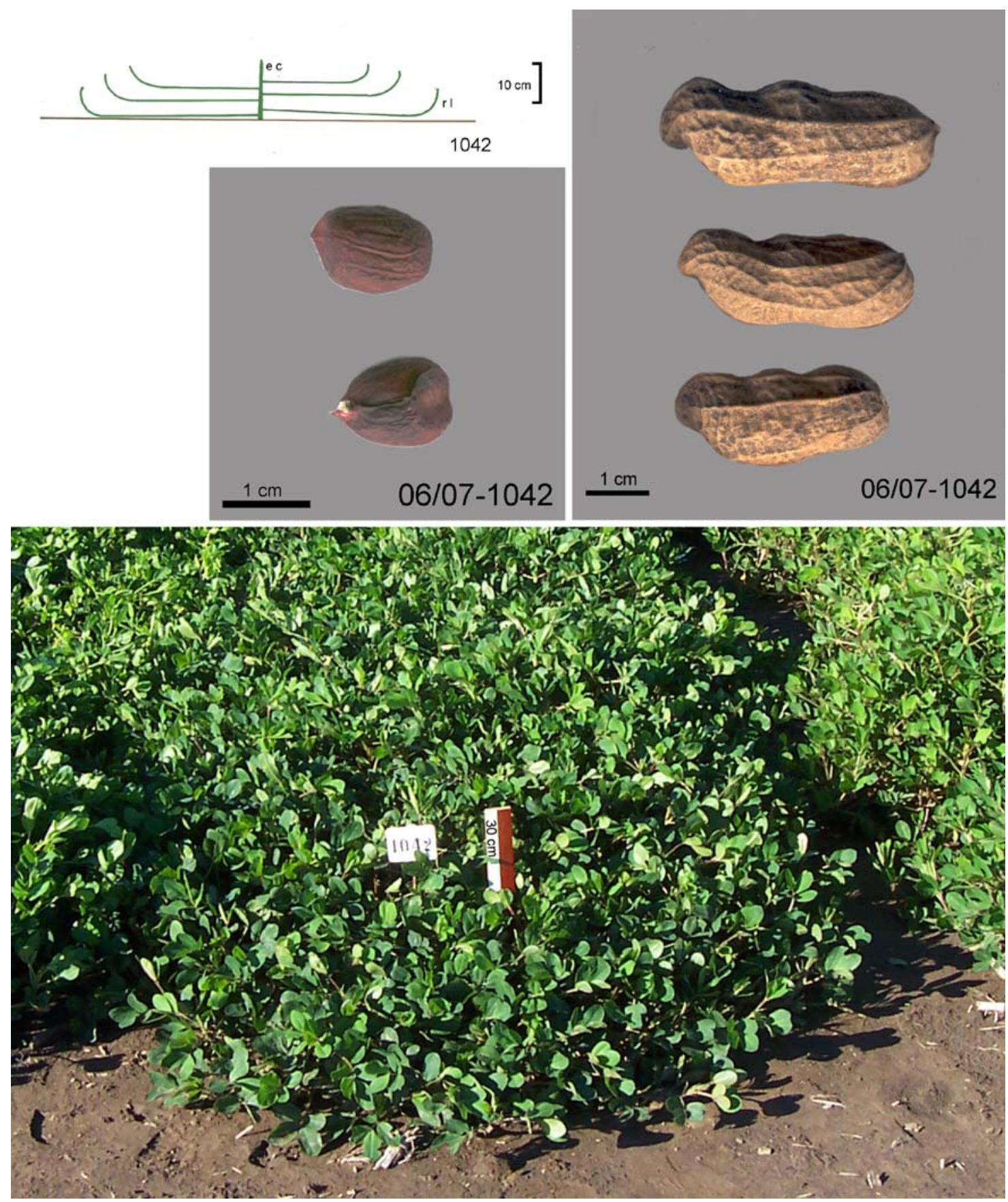


\section{Negro de los Yungas}

Planta cojín, con eje central desarrollado, ramas sinuosas. Brote violáceo.

Cajas con retículo liso, 3 (2) granos negros.

\begin{tabular}{|c|c|}
\hline /07 1046 & 85/86 1996 \\
\hline & 85/86 1997 \\
\hline
\end{tabular}

La Paz, La Paz

La Paz, Sakawaya, S. Yungas
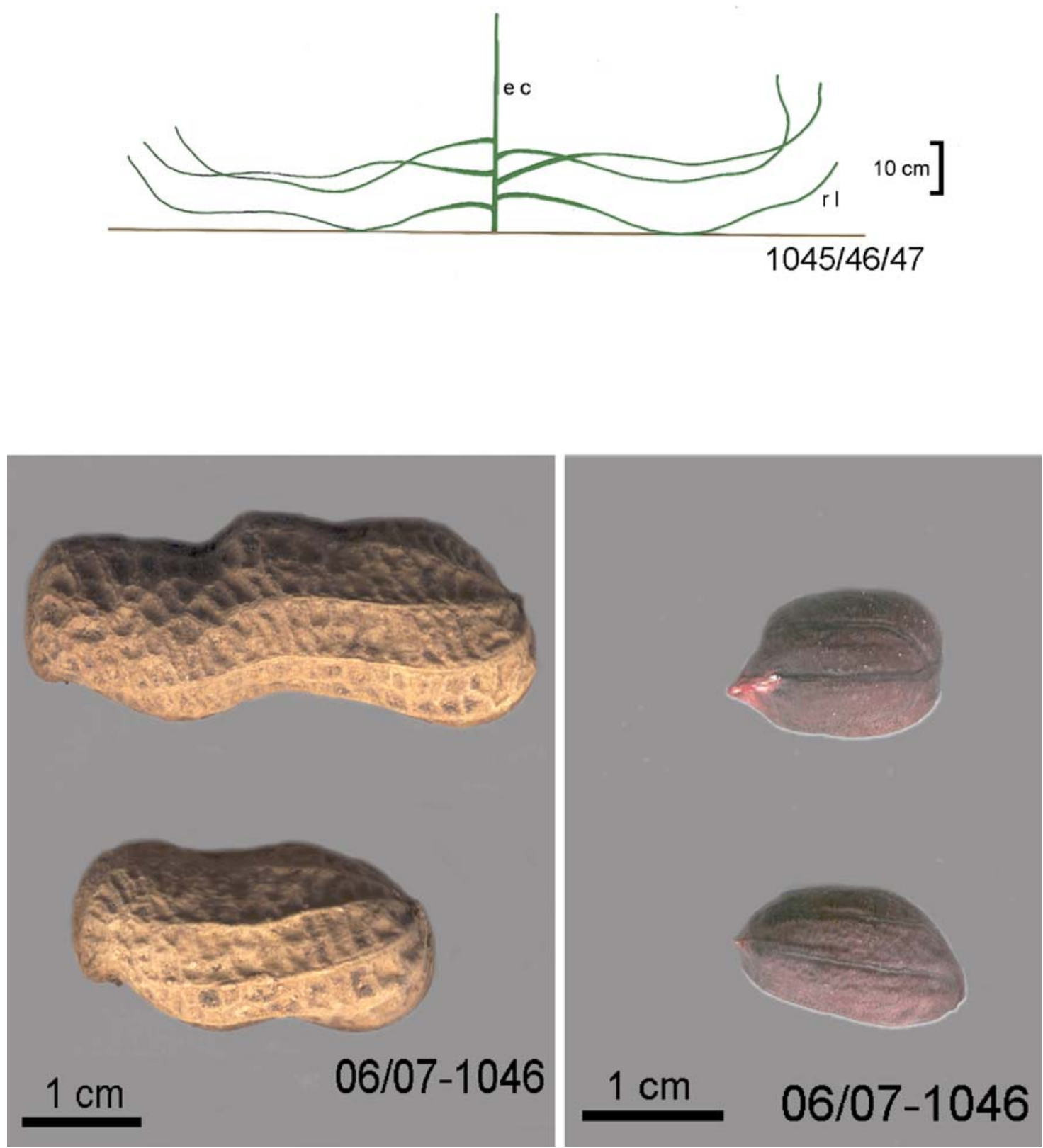
Krapovickas, A.\& al., Las razas de maní de Bolivia

\section{Negro de Aiquile}

Planta rastrera abierta, desparramada, ramas sinuosas. Eje central largo, sinuoso, caído. Hojas mayores y algo más claras. Cerdas hasta el raquis.

Cajas como Sara maní, pero mas chicas.
06/2007 $1050 \quad 85 / 862000$
US $86 \mathrm{BPZ}$
Cochabamba, Aiquile 


\section{Morado Mesa Rancho}

Planta semierecta alta, desparramada, ramas sinuosas. Eje central aparente. Brote violáceo. Cerdas hasta el raquis.

Cajas más o menos lisas. Grano morado.

$\begin{array}{lllll}\text { 06/07 } & 1043 & 85 / 861991-1 & \text { US } 85 \text { BPZ } & \begin{array}{l}\text { Cochabamba, Mesa Rancho } \\ \text { La Paz, San Pablo(Coroico) }\end{array} \\ 1044 & 85 / 861992 & \text { US } 380 \text { BZCoJ } & \end{array}$
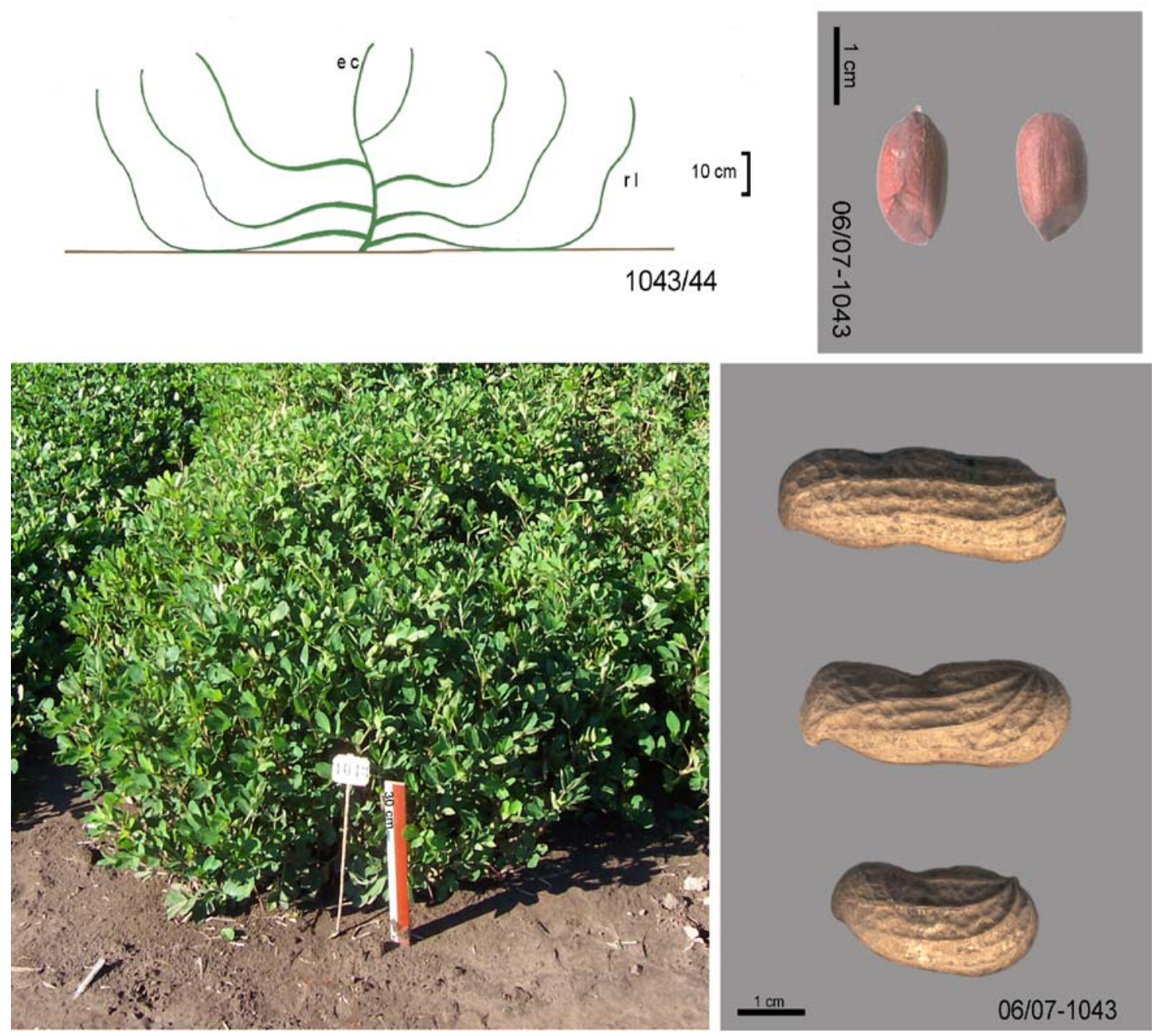


\section{Morado de Santa Cruz}

Planta cojín, achatado, ramificada, desparramada, ramas sinuosas, muy violáceas. Eje central aparente. Hojas grandes. Cerdas hasta el raquis.

Cajas con constricción, estranguladas 2-3 granos morados.
06/07 1045
85/86 1994-1
US 345-1 KSBScCo
Sta. Cruz, Santa Cruz
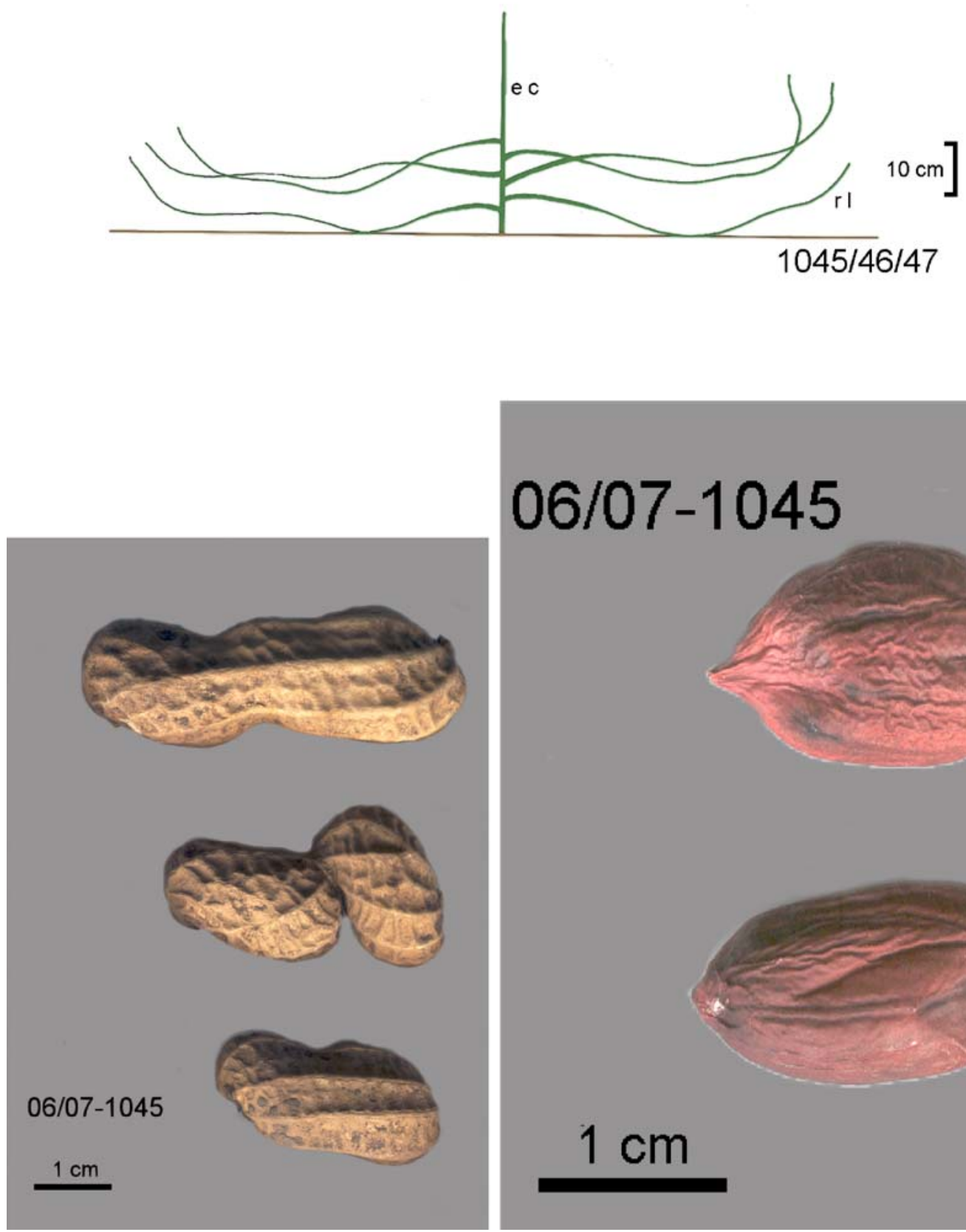

\section{6/07-1045}

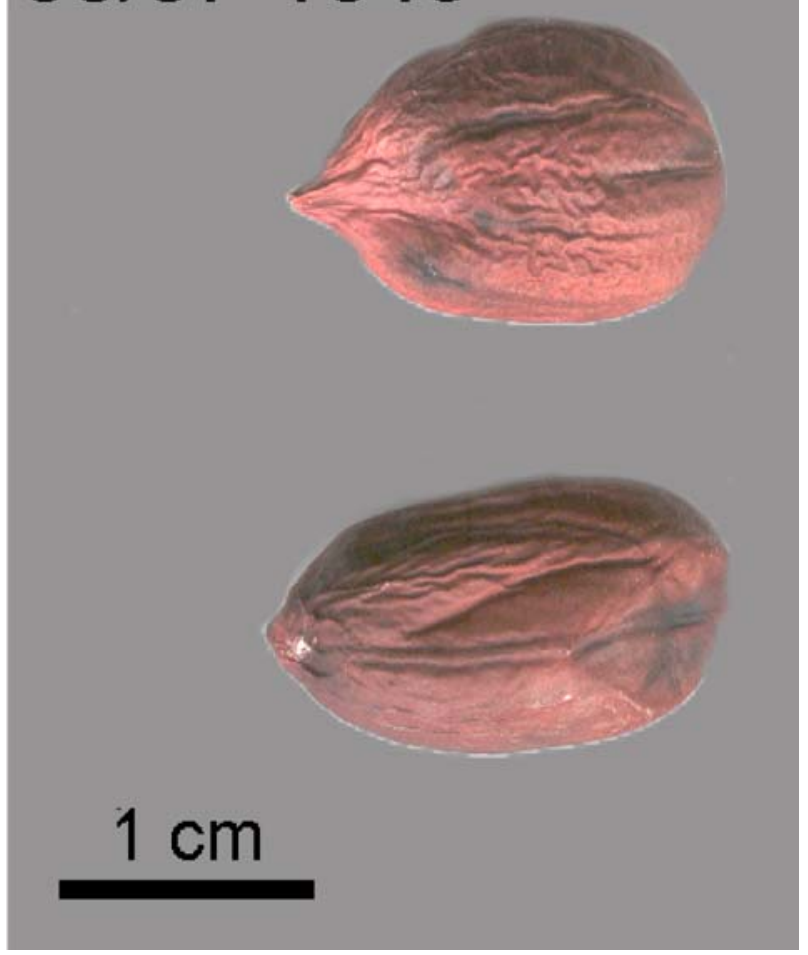




\section{Chaucha morado}

Planta cojín achatada, grande, desparramada, abierta, ramas sinuosas. Tallos morados claros. Eje central aparente. Cerdas hasta el raquis.

Cajas como las de Sara maní, cáscara delgada. Granos morados.

$\begin{array}{lllllll}\text { 06/07 } & 1048 & 85 / 86 & 1999 & \text { RCM } & 1439 \mathrm{~K} & \text { Cochabamba, Cochabamba } \\ & 1049 & 85 / 86 & 2001 & \text { US } & 614 \mathrm{PZi} & \text { Chuquisaca, Sucre }\end{array}$
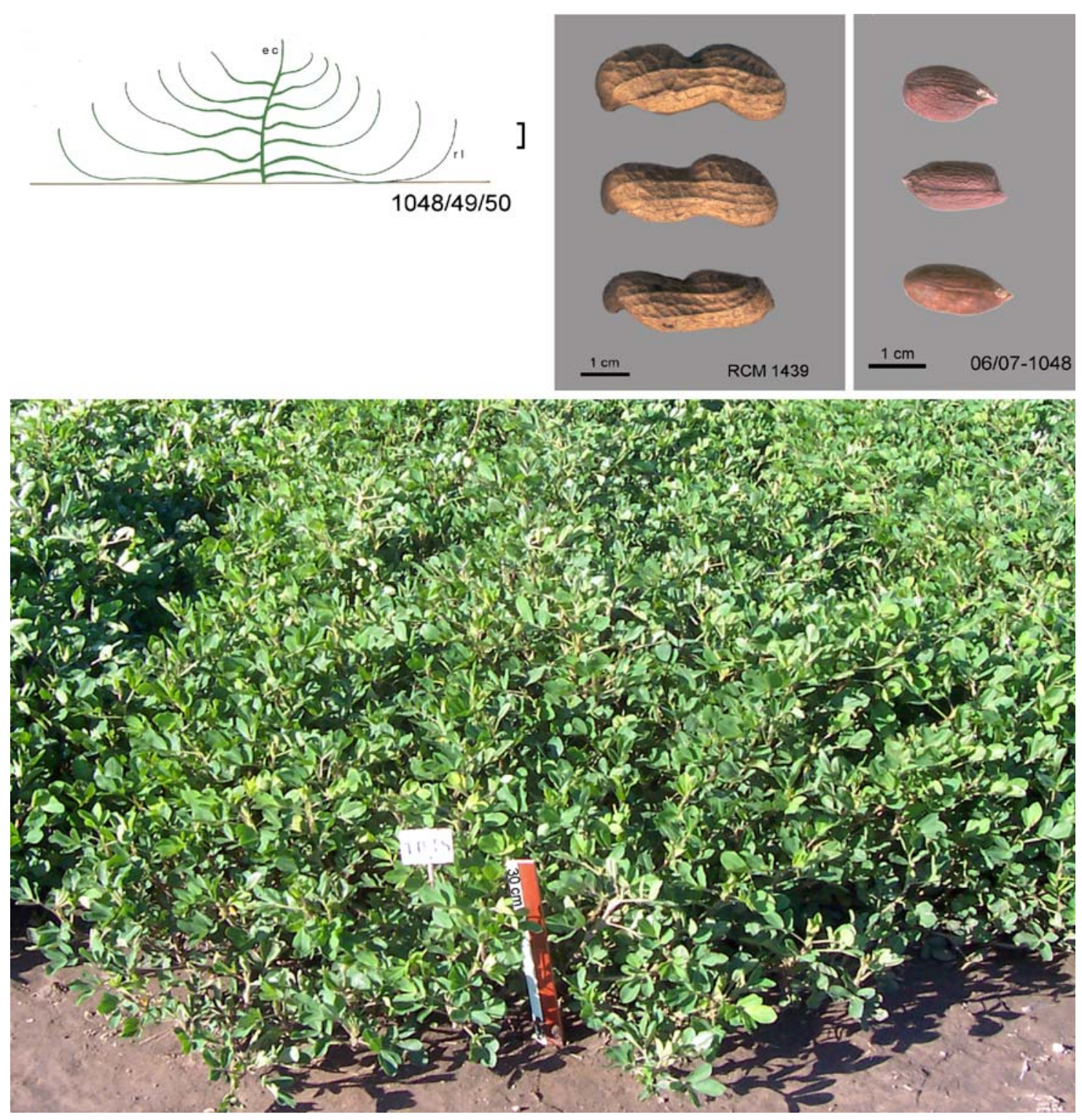

$85 / 862002$

$85 / 862003$

$85 / 862004$

$$
\begin{aligned}
& \text { US } 618 \text { PZi } \\
& \text { ". } 637 \text { " Chaucha morado } \\
& \text { ". } 639-1 \text { ". }
\end{aligned}
$$

Chuquisaca, Sucre

Chuquisaca, Huaca Huasi

Chuquisaca, .Padilla 


\section{Colorado caja redonda}

Planta erecta (bunch), compacta verde claro, eje central no aparente.

Cajas redondas, estranguladas, sin carena. Granos colorado clarete.
06/07 1051
$85 / 861885$
US $808-1$ KSSc
Tarija, Tarija, mercado
1052
$85 / 861888$
US 814-2 KSSc
Tarija, Tarija, mercado
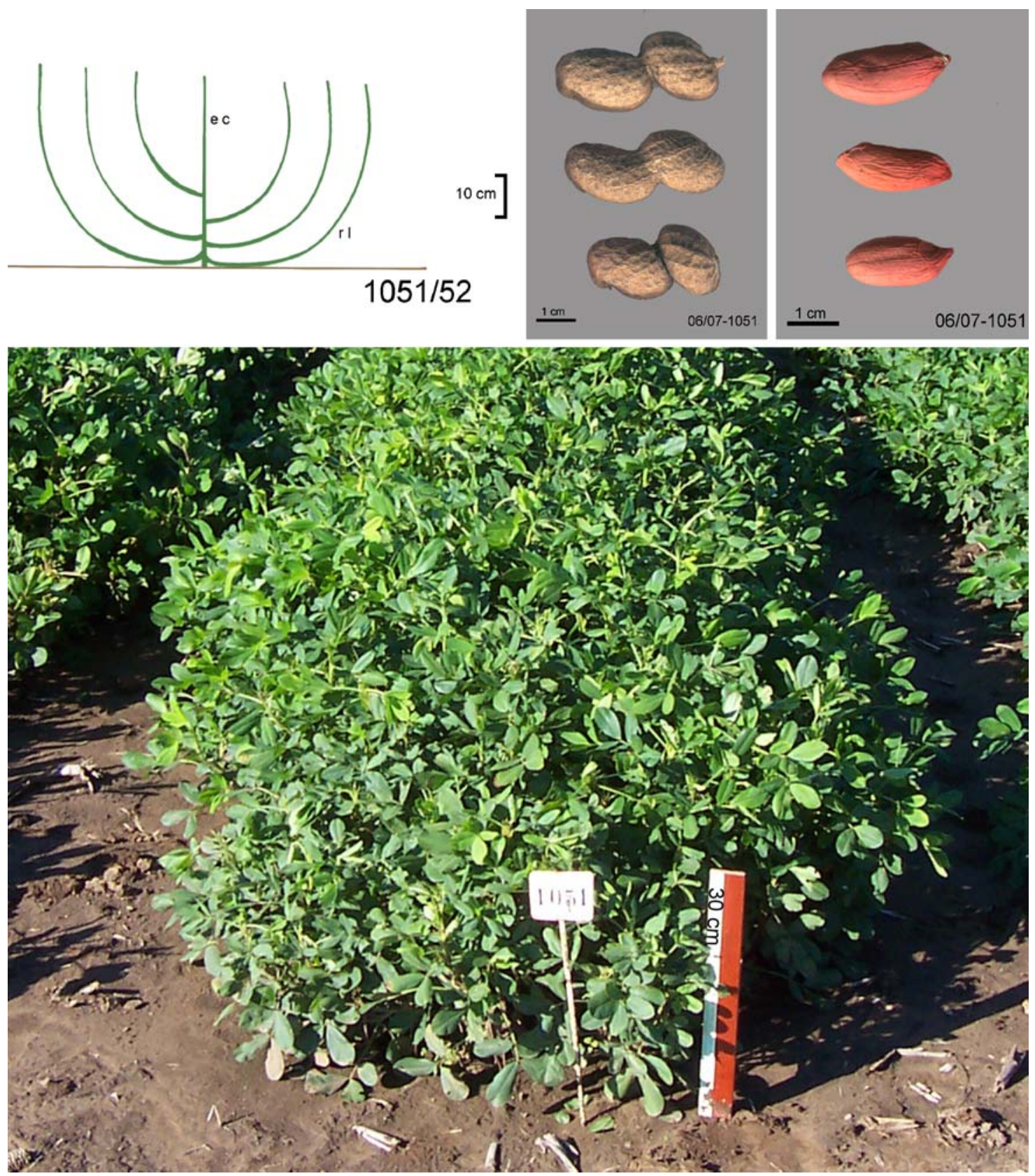

85/86 1906

US $73 \mathrm{BPZ}$

Cochabamba, Mairana 


\section{Colorado revoluto}

Planta erecta compacta, verde oscuro, ramas sinuosas, tallo verde. Folíolos revolutos.

Cajas grandes, redondeadas, estranguladas, reticuladas, sin pico, ni carena, 2-3 granos, colorados.

06/07 1053 85/86 2006 US 52 BPZ Sta. Cruz, Lagunilla, Vallegrande
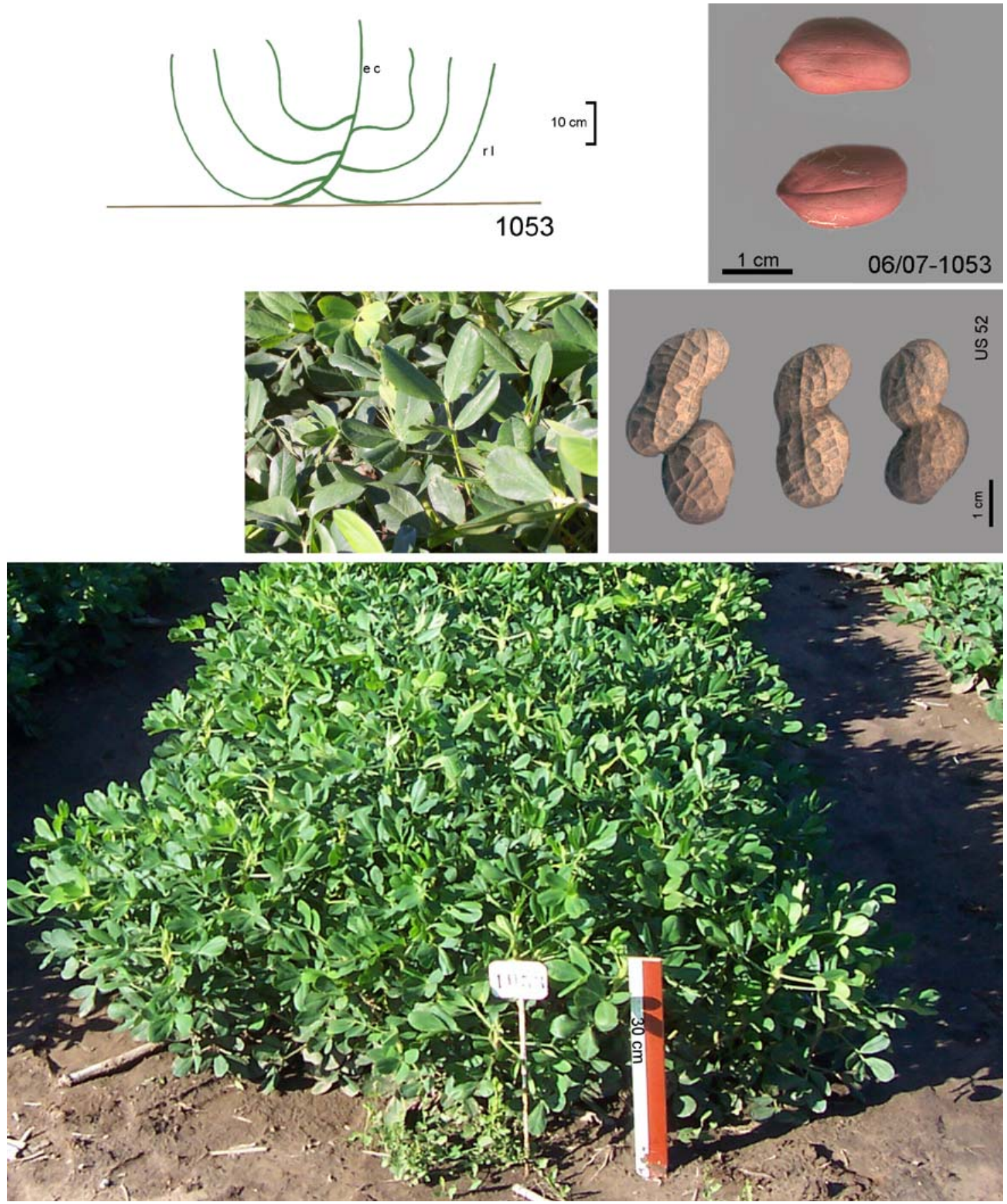


\section{Colorado San Simón}

Planta erecta, compacta, eje central no aparente, ramas rectas.

Cajas con retículo suave, con carena y pico breve, 2- 3 granos colorados.
06/07 1056
$85 / 862030$
RCM $1448 \mathrm{~N}$
06/07 1057
$85 / 862079$
US 302-2 KSBScCo
Sta. Cruz, Mairana
Potosi, Villazón
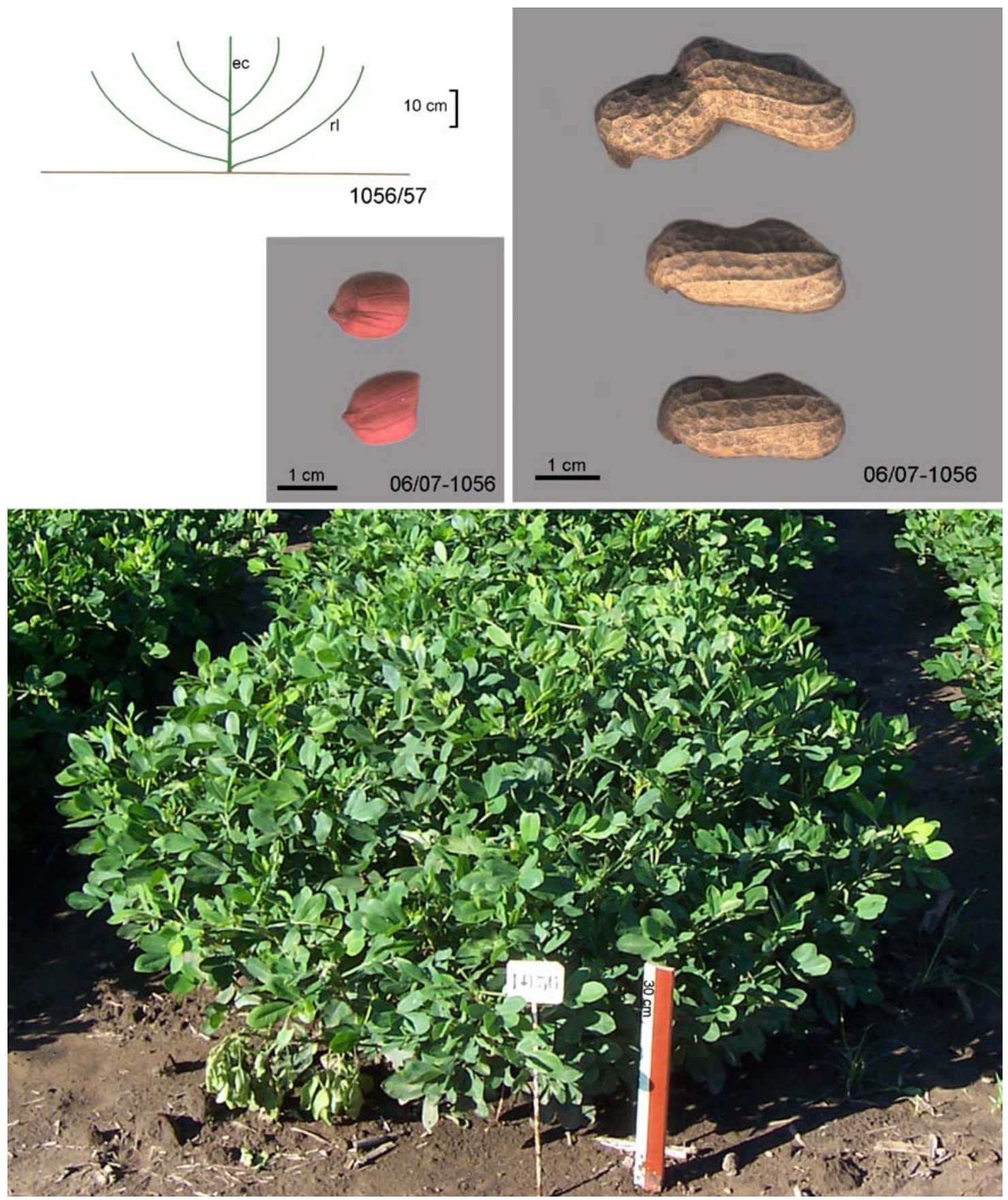
BONPLANDIA 18(2). 2009

\begin{tabular}{|c|c|c|c|}
\hline 85/86 2031 & \multirow{2}{*}{\multicolumn{2}{|c|}{$\begin{array}{r}\text { RCM } 1449 \\
1462\end{array}$}} & $\mathrm{~N}$ \\
\hline 2038 & & & KMoF \\
\hline 2036 & \multirow{8}{*}{ US } & 1484 & ", \\
\hline 2034 & & 1485 & $"$ \\
\hline 2035 & & 1489 & "' \\
\hline 2044 & & 29 & GKBSPSc \\
\hline 2060 & & 37 & " \\
\hline 2061 & & 38 & " \\
\hline 2065 & & 40 & " \\
\hline 2067 & & 41 & " \\
\hline 2040 & & 48 & BPZ \\
\hline 2070 & & 61 & $\mathrm{BPZ}$ \\
\hline 2042 & & 69 & BPZ \\
\hline 2074 & & 73 & BPZ \\
\hline 2075 & & 100 & $\mathrm{BPZ}$ \\
\hline 2076 & & 101 & BPZ \\
\hline 2080 & & $305-2$ & KSBScCo \\
\hline 2081 & & $313-2$ & "' \\
\hline 2082 & & $318-2$ & " \\
\hline 2083 & & 322 & " \\
\hline 2084 & & $323-2$ & "' \\
\hline 2085 & & $384-2$ & BZCo \\
\hline 2087 & US & $823-2$ & KSSc \\
\hline 2088 & & $824-2$ & "' \\
\hline 2089 & & $826-2$ & " \\
\hline 2090 & & $828-2$ & " \\
\hline 2091 & & $829-2$ & " \\
\hline 2092 & & $831-2$ & " \\
\hline 2093 & & $832-4$ & $"$ \\
\hline 2094 & & 833-2 & "' \\
\hline 2095 & & $840-2$ & " \\
\hline 2096 & & $855-3$ & " \\
\hline
\end{tabular}

Sta. Cruz, Mairana

Cochabamba, Cochabamba

Tarija, Hualcalques

Tarija, Entre Ríos

Tarija, Villa Montes

Sta. Cruz, Gutierrez

Sta. Cruz, Saavedra

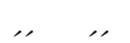

Sta. Cruz, Yerba Buena

Sta. Cruz, Trigal

Sta. Cruz, Samaipata

Sta. Cruz, Mairana

La Paz, La Paz

La Paz, La Paz

Potosi, Villazon

$\begin{array}{ll}\text { ", } & \text { ", } \\ \text {,. } & \text { ". } \\ \text {,. } & \text { ". }\end{array}$

La Paz, Coroico

Tarija, Entre Ríos

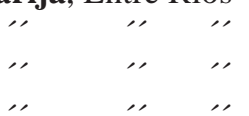

Tarija, S. Simón

$\begin{array}{cc}\text {,. } & \text { Entre Ríos } \\ \text {,. } & \text {,. } \\ \text {,. } & \text { Villa Montes } \\ \text {,. } & \text {,. }\end{array}$




\section{Colorado carenado}

Planta erecta compacta, alta, verde oscuro. Con sarna.

Cajas carenadas, dos a tres granos, colorado clarete.

$\begin{array}{lllll}\text { 06/07 } & 1054 & \text { 85/86 2013 } & \text { RCM 1467 K } & \begin{array}{l}\text { Sta Cruz, Samaipata } \\ \text { Cochabamba, Cochabamba }\end{array} \\ & 1055 & 85 / 862015 & \text { US 98 BPZ } & \text { C }\end{array}$
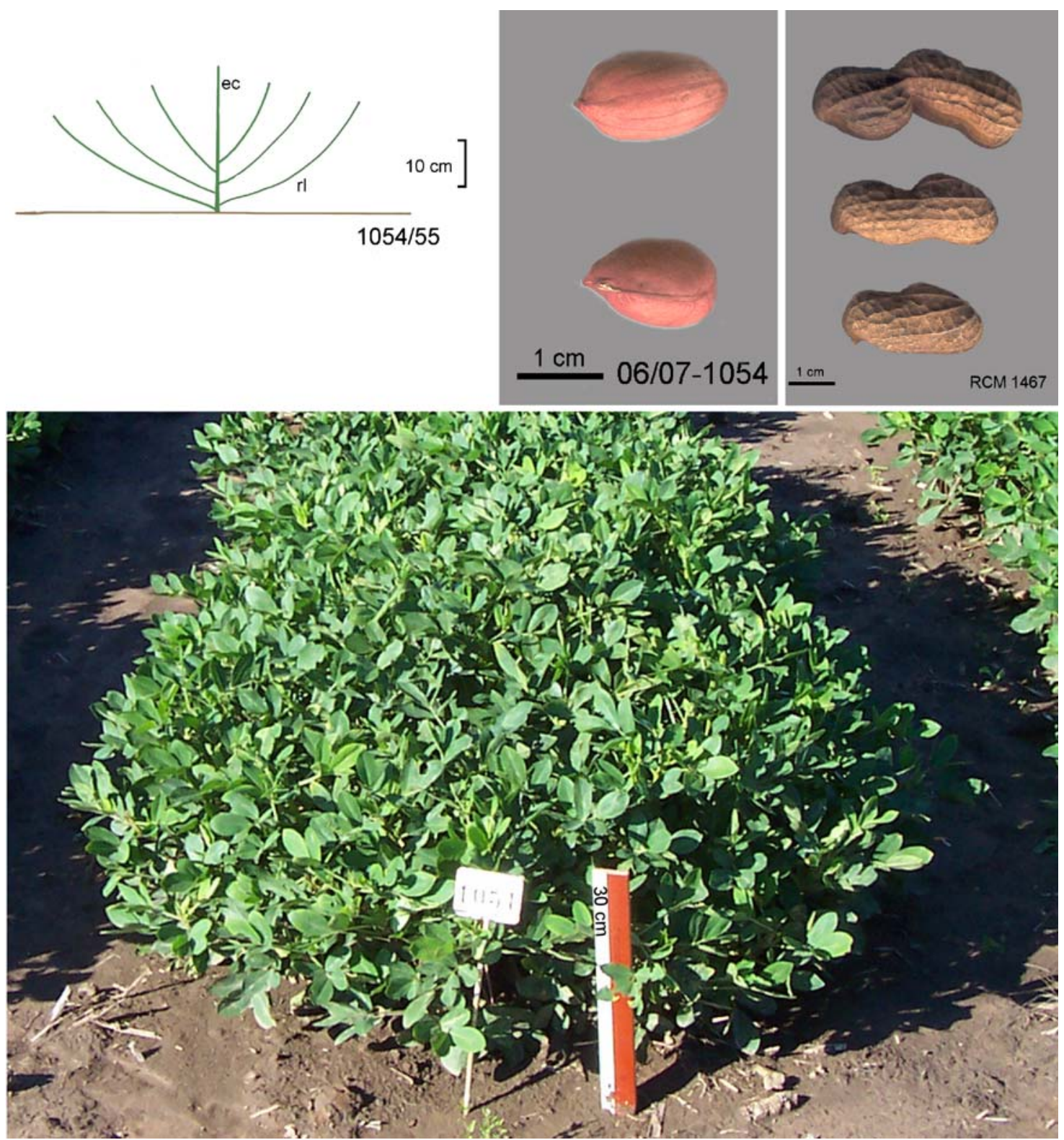

$85 / 862020$

$85 / 862022$

$85 / 862024$

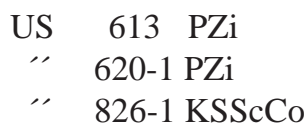

\section{Chuquisaca, Sucre}

Tarija, Entre Ríos 


\section{Salmón carenado}

Planta alta compacta verde oscuro, ramas sinuosas. Con sarna.

Cajas con constricción y pico, 2 granos salmón.
06/07 1060
1061
85/86 2007
$85 / 862009$
US 23 GKSBPSc
US $73 \mathrm{BPZ}$

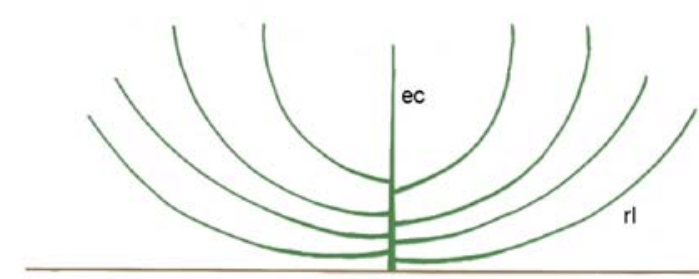

$1060 / 61$

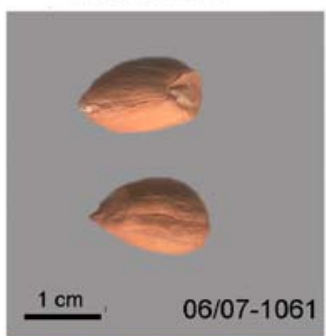

$1 \mathrm{~cm}$

06/07-1061
Tarija, Villa Montes

Sta. Cruz,Villa Victoria, Mairana

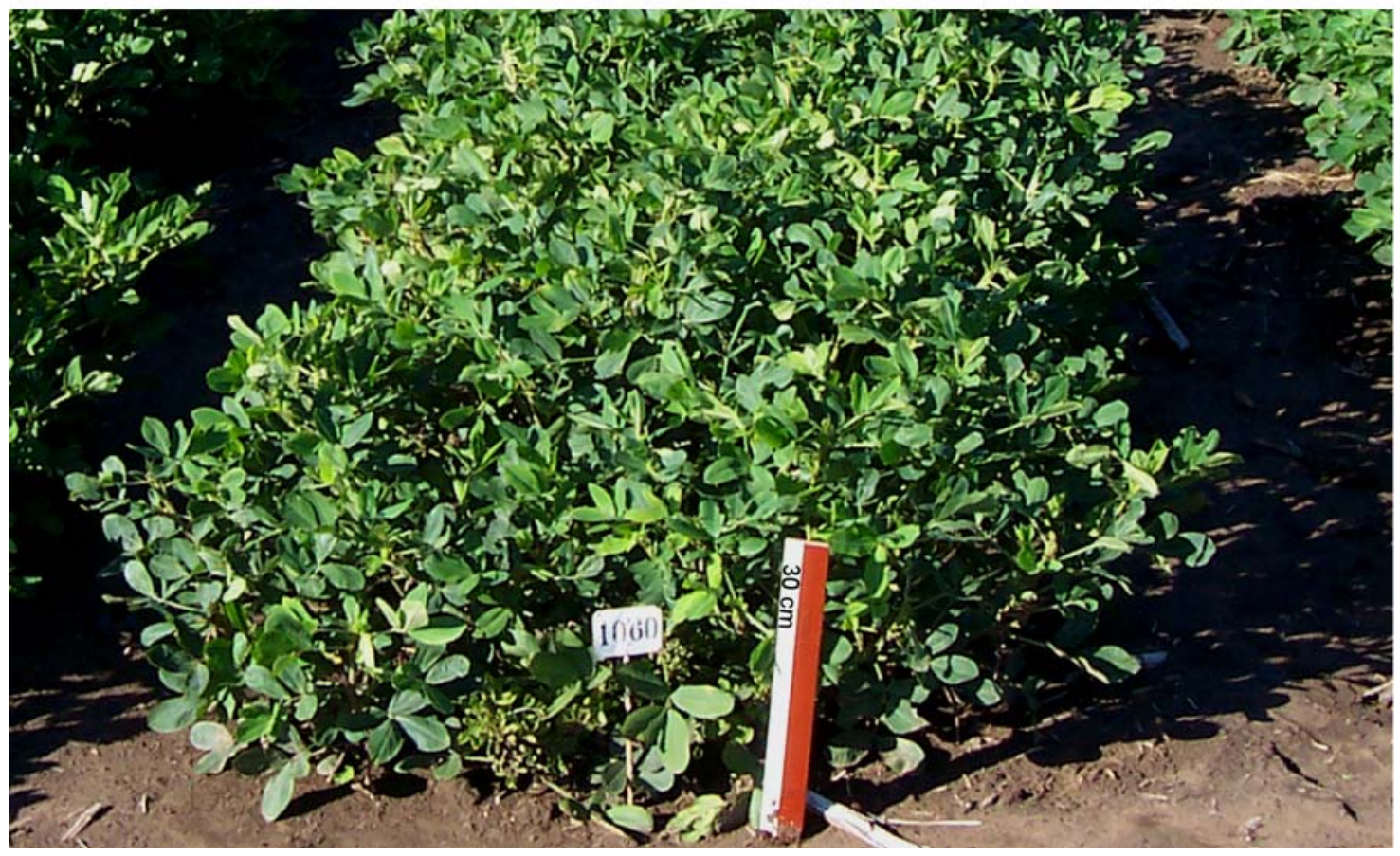

85/86 2008

85/86 2010

85/86 1891

\section{US 31 GKSBPSc 815 KSSc 823-3}




\section{Overo negro}

Planta rastrera, eje central aparente. Con cerdas. Brote violáceco.

Cajas redondeadas, reticuladas, con constricción mediana, sin pico, sin quilla. 2 granos negros y albos.

06/07 1013 85/86 1720 US 217 GKSPSc Beni, Huacaraje
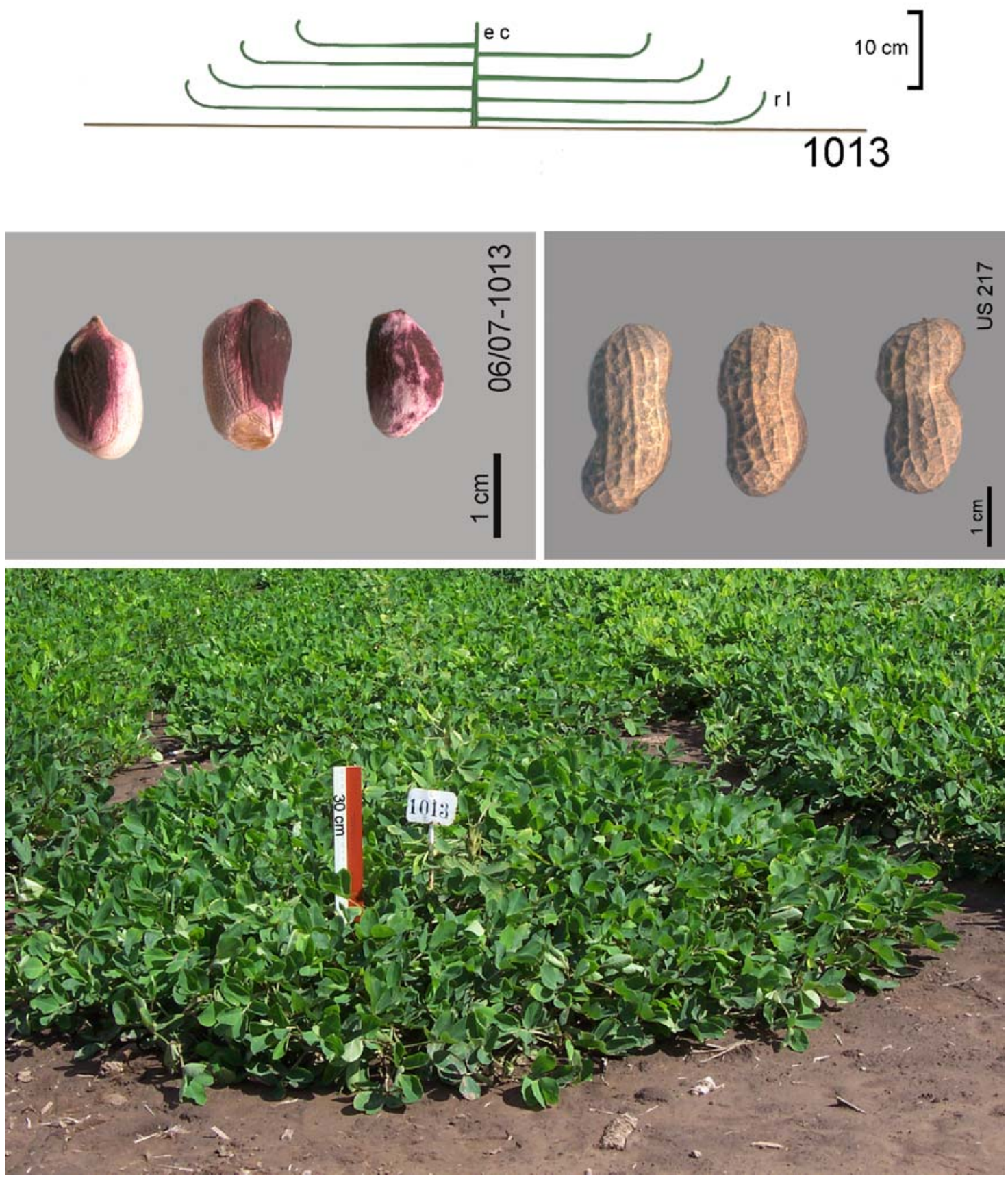


\section{Overo alazán rastrero}

Planta rastrera, eje central aparente. Con cerdas hasta en el raquis.

Cajas grandes, redondeadas, con constricción marcada, con quilla. Granos alazán más albo.
06/7 1014 85/86 1722
1015 85/86 2204-1
US 310 KSBScCo
304
Potosi, Villazón

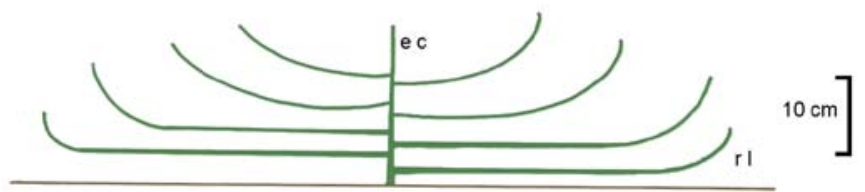

$1014 / 15$
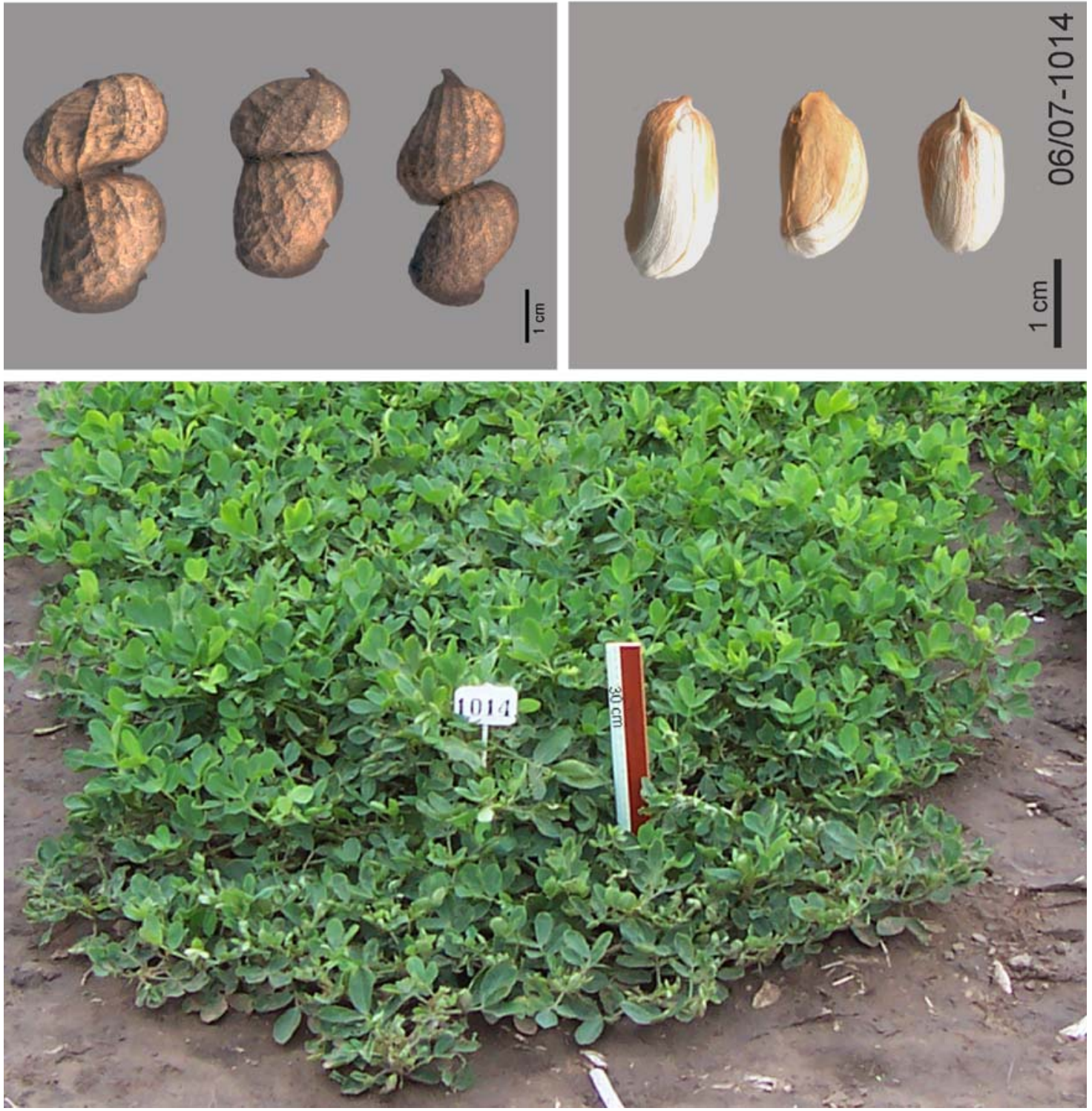


\section{Overo rastrero}

Planta rastrera, grande, eje central aparente.

Cajas grandes, retículo marcado, con carena. 2 granos overos, colorado y albo.

$\begin{array}{lllll}\text { 06/07 } 1016 & \text { 85/86 2205 } & \text { US 315-1 } & \text { KSBScCo } & \text { Potosi, Villazón } \\ & 85 / 862215-1 & \text { US 620 } & \text { PZi } & \text { Chuquisaca, Sucre }\end{array}$
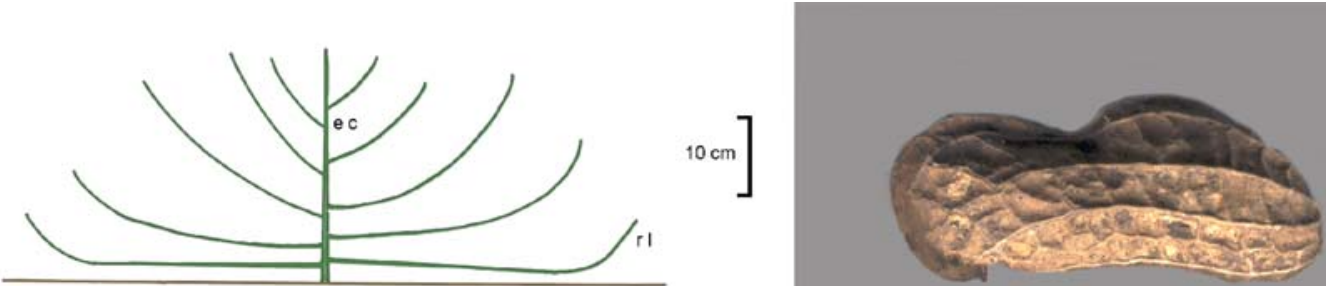

1016
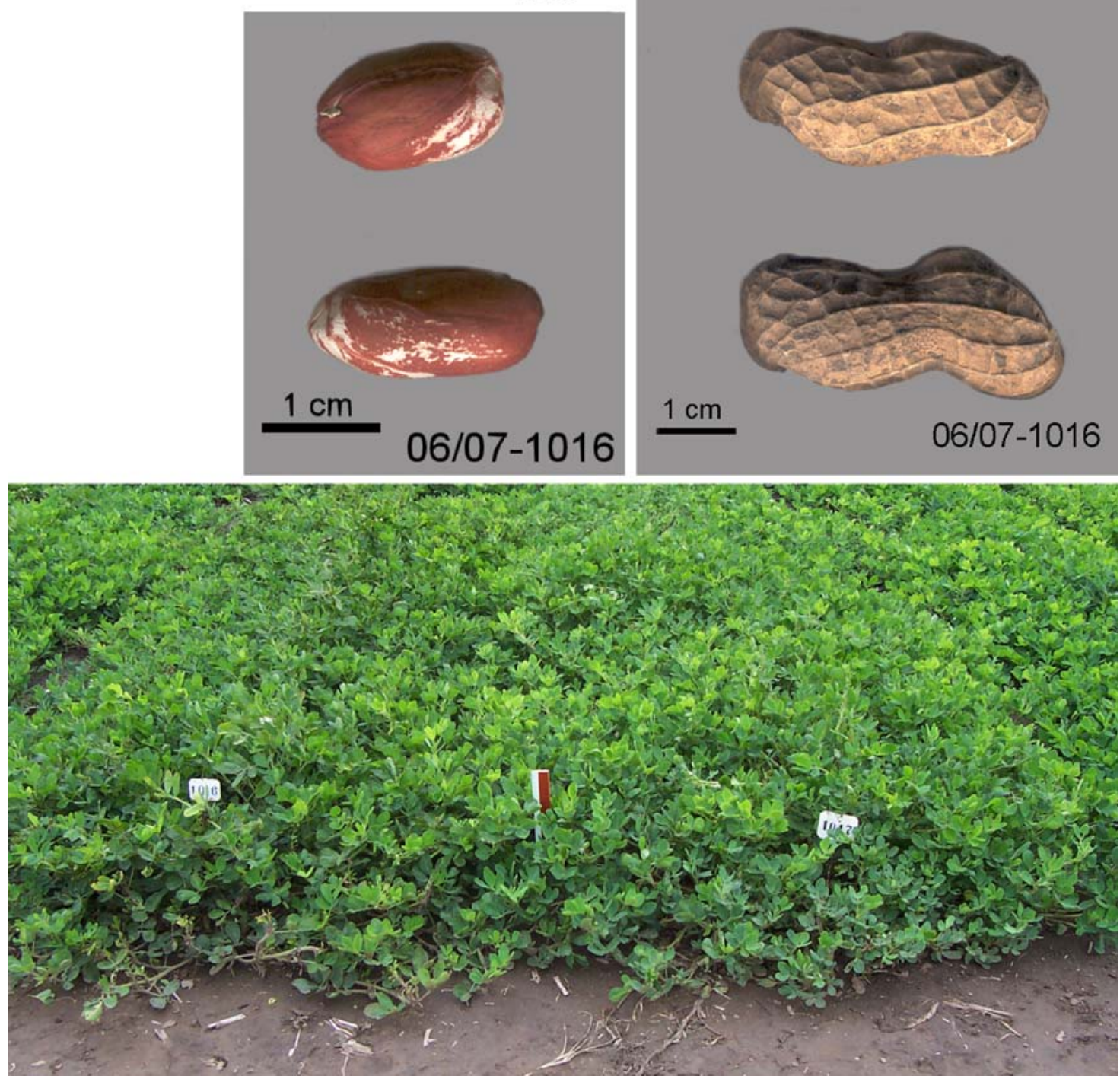
BONPLANDIA 18(2). 2009

\section{Rastrero veteado}

US 105 BPZ

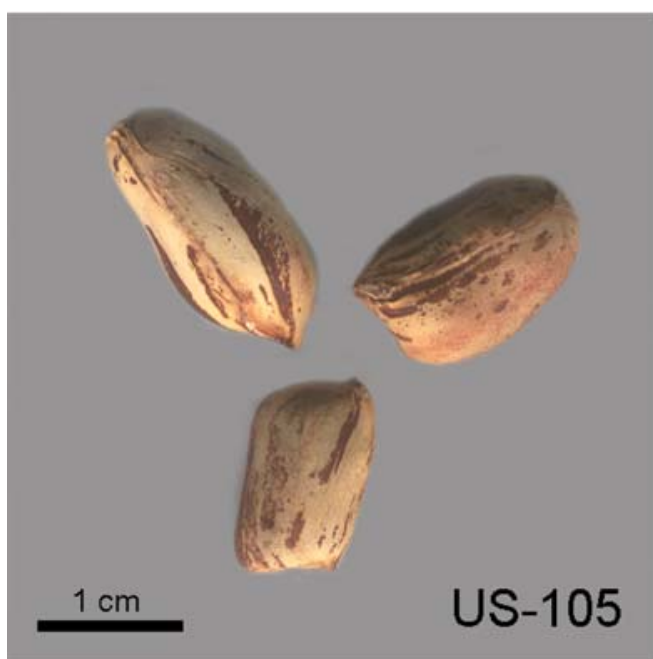

La Paz, S.Yungas, Sanani

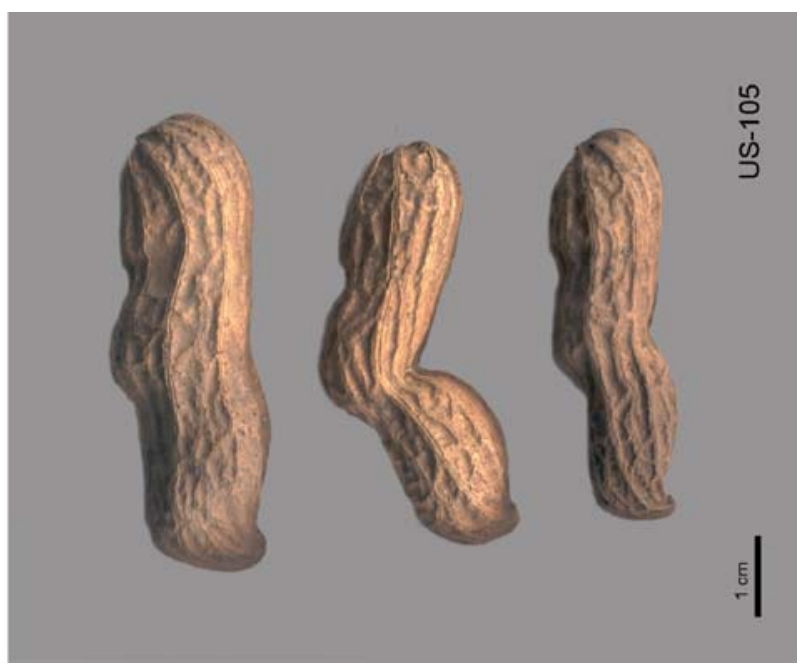




\section{Overo cojín carenado}

Planta cojín grande, eje central algo aparente, apenas se tocan.

Cajas reticuladas, con carena marcada, estranguladas a veces. 2 granos, colorado fuerte más albo.

\begin{tabular}{|c|c|c|c|c|}
\hline 06/07 & $\begin{array}{l}1075 \\
1076\end{array}$ & $\begin{array}{l}85 / 862206 \\
85 / 862207\end{array}$ & $\begin{array}{cc}\text { US } 322 & \text { KSBScCo } \\
\text { US } 338 & \text { ", }\end{array}$ & $\begin{array}{l}\text { Potosi, Villazón } \\
\text { Sta. Cruz, Santa Cruz }\end{array}$ \\
\hline
\end{tabular}
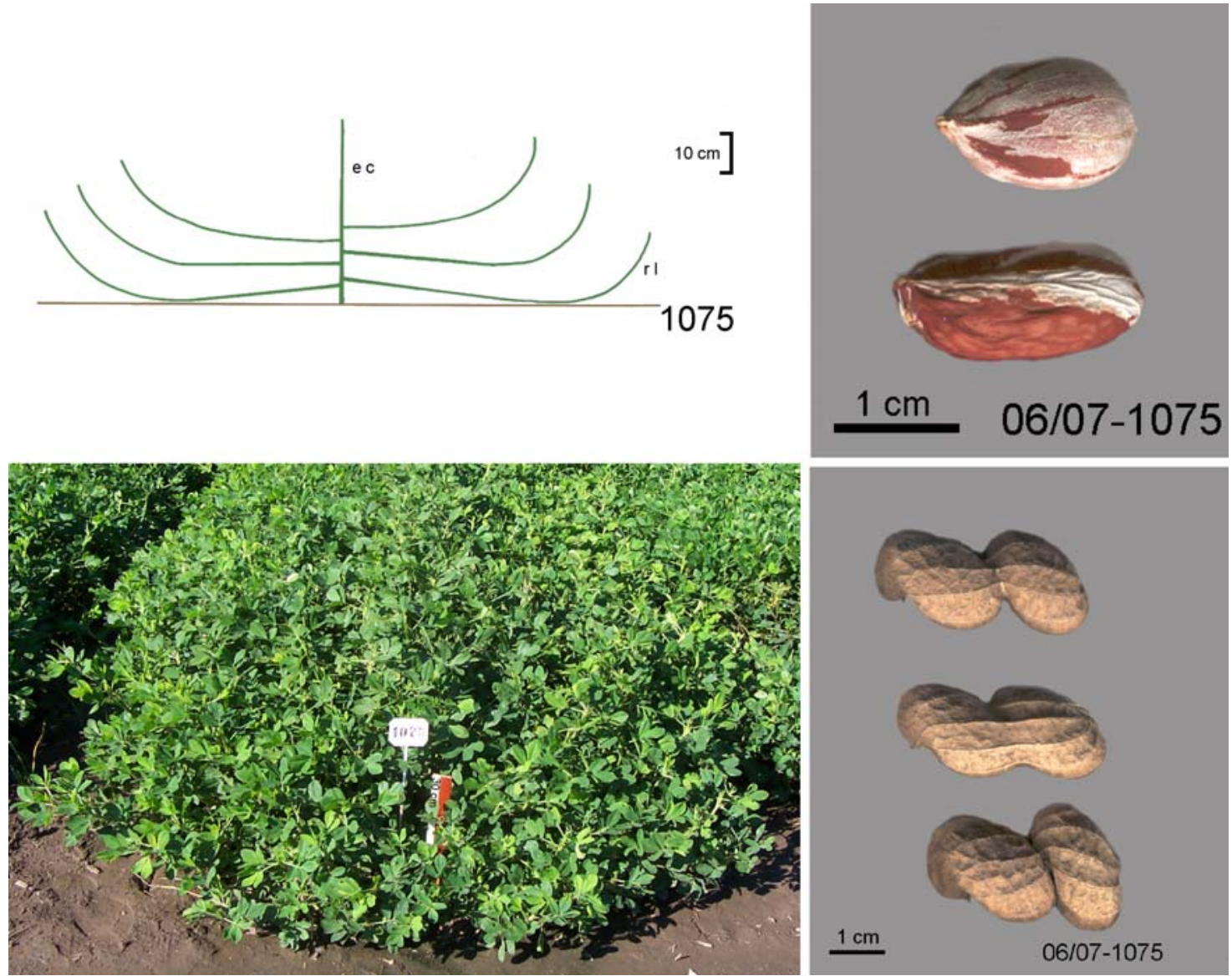

85/86 2281

$85 / 862344$

85/86 2209

$85 / 862210$

$85 / 862216$

$85 / 862213$

$85 / 862214$

85/86 2217

85/86 2218

$85 / 862219$

$85 / 862220$

85/86 2221

\begin{tabular}{|c|c|}
\hline US & 38 GKBSPSc \\
\hline & 318 KSBScCo \\
\hline , & 359 BZCo \\
\hline & $362 \quad \cdots$ \\
\hline & $613 \mathrm{PZi}$ \\
\hline & $617-1^{\prime \prime}$ \\
\hline & $620 \quad$ "' \\
\hline & $820 \mathrm{KSSc}$ \\
\hline & $826-1$ "' \\
\hline & 829-1 "' \\
\hline & 840-1 " \\
\hline & 841 \\
\hline
\end{tabular}

Sta Cruz, Est. Exp. Saavedra Potosi, Villazón

La Paz, La Paz

Chuquisaca, Sucre

Tarija, Tarija

$$
\begin{array}{ll}
\text {,. } & \text { Entre Ríos } \\
\text {,. } & \text { San Simón } \\
& \text { Villa Montes }
\end{array}
$$




\section{Overo alazán}

Planta cojín erecto, eje central no aparente, ramas sinuosas.

Cajas reticuladas, estranguladas, con carena y pico. 2 granos, alazán + rosadito.

$\begin{array}{llllll}06 / 07 & 1068 & 85 / 862187 & \text { US 832-2 } & \text { KMoF } & \text { Tarija, Entre Ríos } \\ & 1069 & 85 / 862228 & \text { US 616 } & \text { PZi } & \text { Chuquisaca, Sucre }\end{array}$
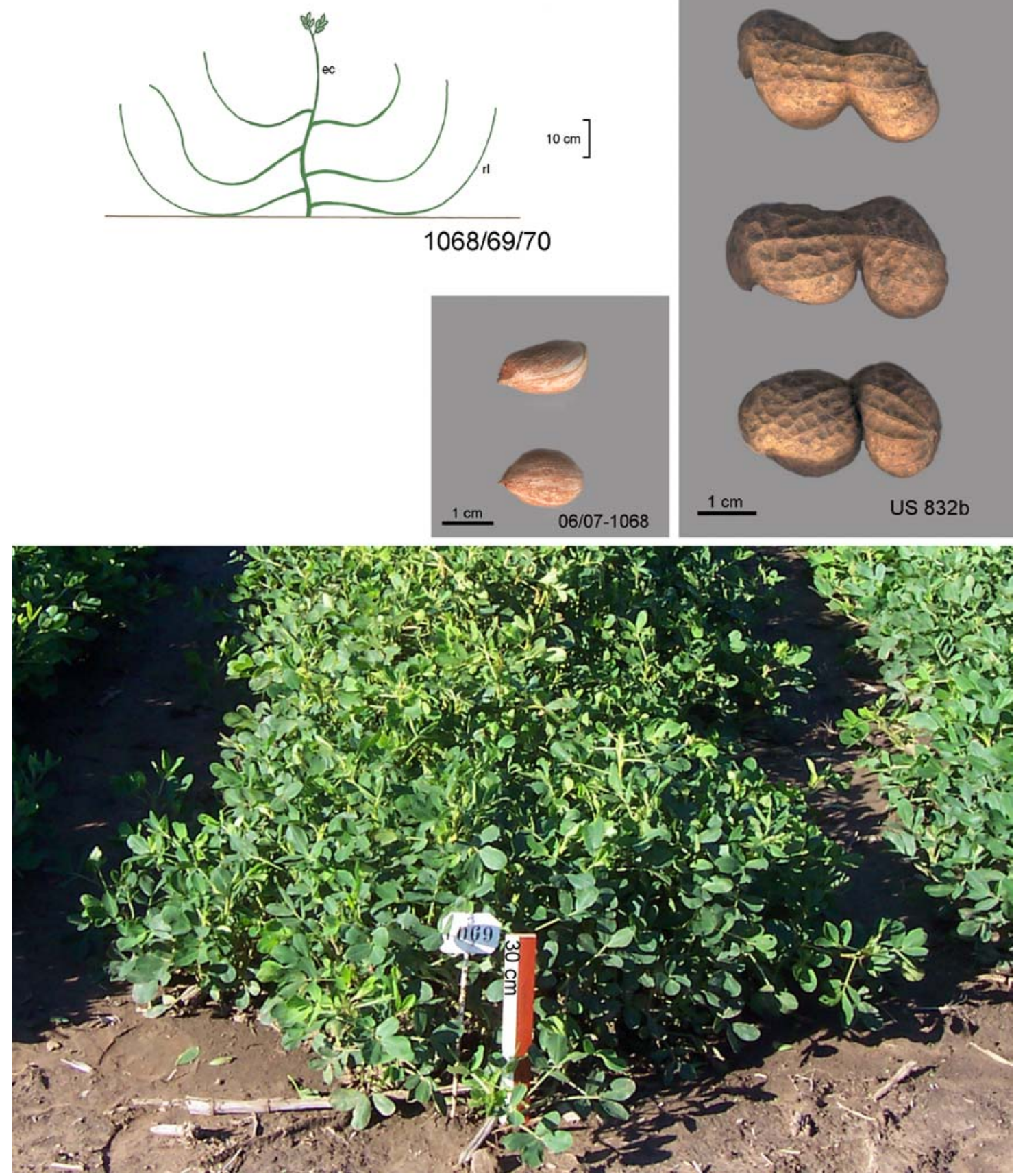


\section{Bayo Americano}

Planta bunch grande. Cojín de ramas sinuosas.

Cajas grandes (tipo pepón), redondeadas, retículo marcado, estranguladas, sin carena. 2- (3) granos rosados y rosado claro.

$\begin{array}{rrrrr}06 / 07 & 1066 & 85 / 862168 & \text { RCM } 1473 \mathrm{KMoF} & \text { Tarija, Tarija } \\ 1067 & 85 / 862169 & \text { RCM } 1482 \mathrm{KMoF} & \text { Tarija, Tarija }\end{array}$

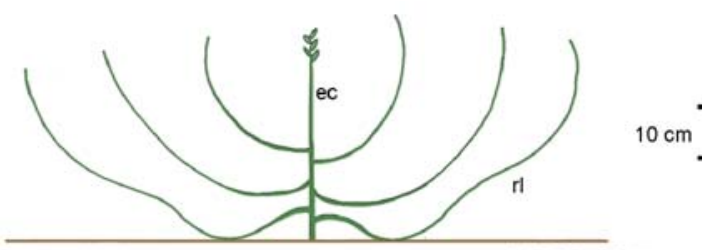

$1066 / 67$

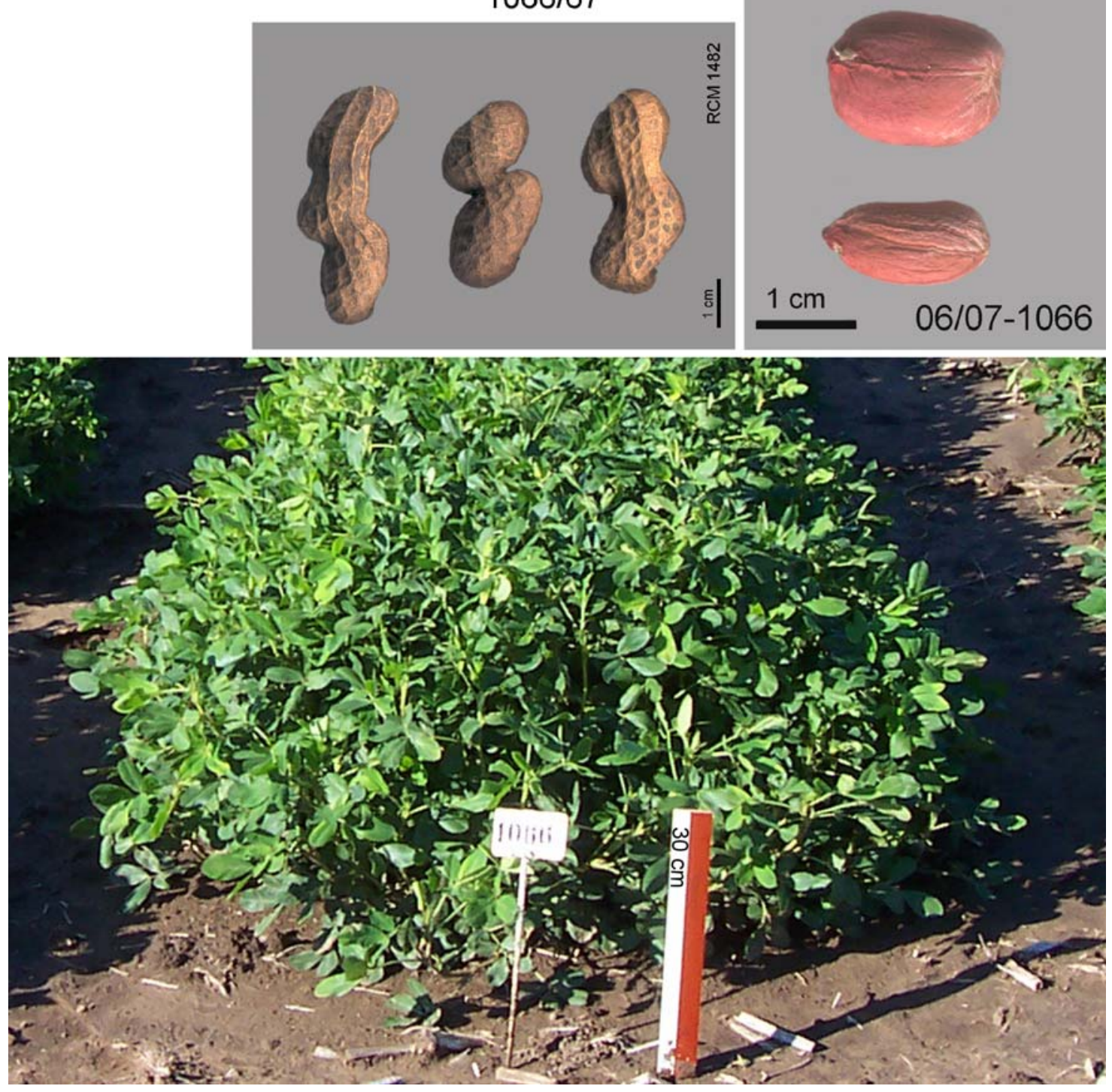


BONPLANDIA 18(2). 2009

\begin{tabular}{|c|c|c|c|}
\hline 85/86 2156 & \multicolumn{2}{|c|}{ RCM 1487} & KMoF \\
\hline 85/86 2170 & US & 21 & GKBSPSc \\
\hline 85/86 2224-1 & " & 97 & $\mathrm{BPZ}$ \\
\hline 85/86 2172 & " & 316 & KSBScCo \\
\hline $85 / 862173$ & " & 317 & \\
\hline 85/862174 & " & 365 & BZCo \\
\hline 85/86 2225 & US & 613 & PZi \\
\hline 85/86 2223-1 & US & 616 & $\mathrm{PZi}$ \\
\hline 85/86 2179 & $"$ & 806 & KSSc \\
\hline 85/86 2180 & " & 807-1 & "' \\
\hline $85 / 862181$ & " & 808-1 & " \\
\hline 85/86 2182 & " & 810-2 & " \\
\hline $85 / 86$ & " & $811-2$ & " \\
\hline 85/86 2183 & " & 813-1 & " \\
\hline $85 / 862184$ & $"$ & 814-1 & " \\
\hline 85/86 2185 & $"$ & 816-2 & $"$ \\
\hline 85/86 2186 & " & 818-1 & " \\
\hline $85 / 862230$ & " & 819 & KSSC \\
\hline 85/86 2226 & $"$ & 820 & $"$ \\
\hline 85/86 2163 & " & $833-4$ & $"$ \\
\hline 85/86 2188 & " & 834-1 & $"$ \\
\hline 85/86 2189 & $"$ & 855-1 & $"$ \\
\hline
\end{tabular}

Tarija, Hualcalques

Tarija, Caraparí

Cochabamba, Cochabamba

Potosi, Villazón

La Paz, La Paz

Chuquisaca, Sucre

Chuquisaca, Sucre

Tarija, Tarija

$$
\begin{array}{ll}
\text {, } & \text {,. } \\
\text {, } & \text { ", } \\
\text {, } & \text {,. } \\
\text {, } & \text {, } \\
\text {, } & \text {,. }
\end{array}
$$

Tarija, Tarija
"
" " Entre Ríos
". Entre Ríos
, Villa Montes 


\section{Overo valluno}

Planta bunch grande, $1 \mathrm{~m}$ de diámetro, ramas ramas rectas. Eje central no aparente a algo aparente.

Cajas reticuladas, redondeadas, sin carena ni pico. 2 granos, colorado clarete, rosado y rosado claro.
06/07 1062
1063
$85 / 862122$
$85 / 862129$
$\begin{array}{ll}\text { US } 48 & \text { BPZ } \\ \text { US } 53 & \text { BPZ }\end{array}$

Sta Cruz, Yerba Buena

Sta. Cruz, Mataral
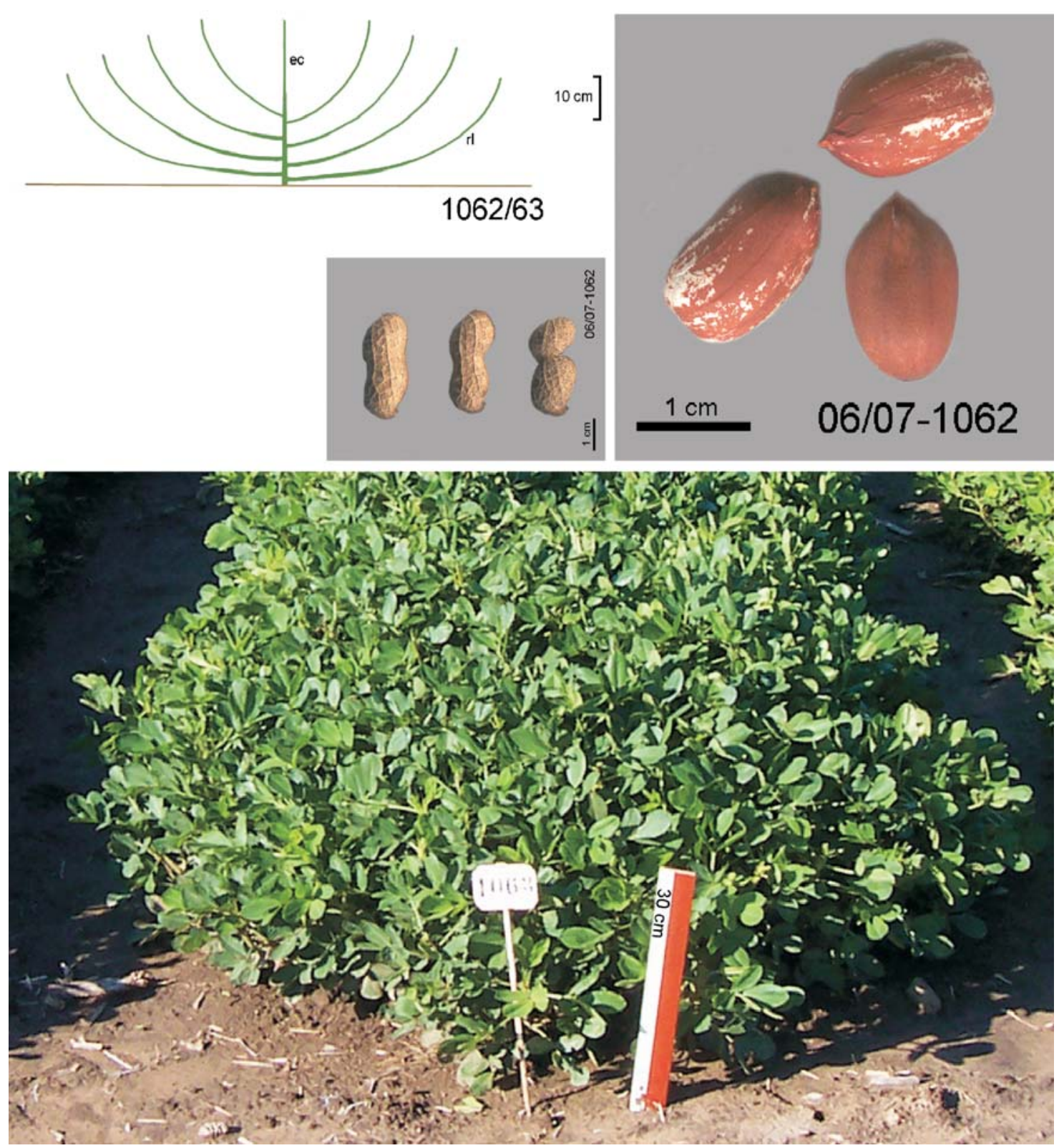
BONPLANDIA 18(2). 2009

\begin{tabular}{|c|c|c|c|}
\hline 85/86 2126 & US & 50 & BPZ \\
\hline 85/86 2127 & & 51 & \\
\hline 85/86 2128 & $"$ & 52 & \\
\hline 85/86 2132 & " & 57 & \\
\hline 85/86 2134 & " & 59 & ' \\
\hline 85/86 2137 & "' & 60 & ' \\
\hline 85/86 2131 & " & 65 & , \\
\hline 85/86 2138 & " & 66 & \\
\hline $85 / 862140$ & " & 68 & \\
\hline 85/86 2141 & $"$ & 70 & \\
\hline 85/86 2142 & " & 71 & " \\
\hline 85/86 2143 & " & 73 & \\
\hline 85/86 2144 & " & 74 & \\
\hline 85/86 2145 & ' & 75 & \\
\hline 85/86 2147 & & 76 & \\
\hline 85/86 2149 & & 348 & $"$ \\
\hline $85 / 862150$ & $"$ & 357 & $"$ \\
\hline
\end{tabular}

Sta. Cruz, Puente del Mataral

"

" Lagunilla

" Vallegrande

" Trigal

" "

" Cochabambita

" "

"

" " La Tuna

" Monteagudo

" Mairana

", ",

" Valle Abajo

" San Isidro

" Santa Cruz

La Paz, La Paz 


\section{Overo colorado}

Planta semierecta, má o menos compacta, sin cerdas.

Cajas con carena, poco pico, sin estrangulamiento, con 2 (3) granos, colorados más rosado.
06/07 1073
85/86 2196-2
US 833-5 KSSc
Tarija, Entre Ríos
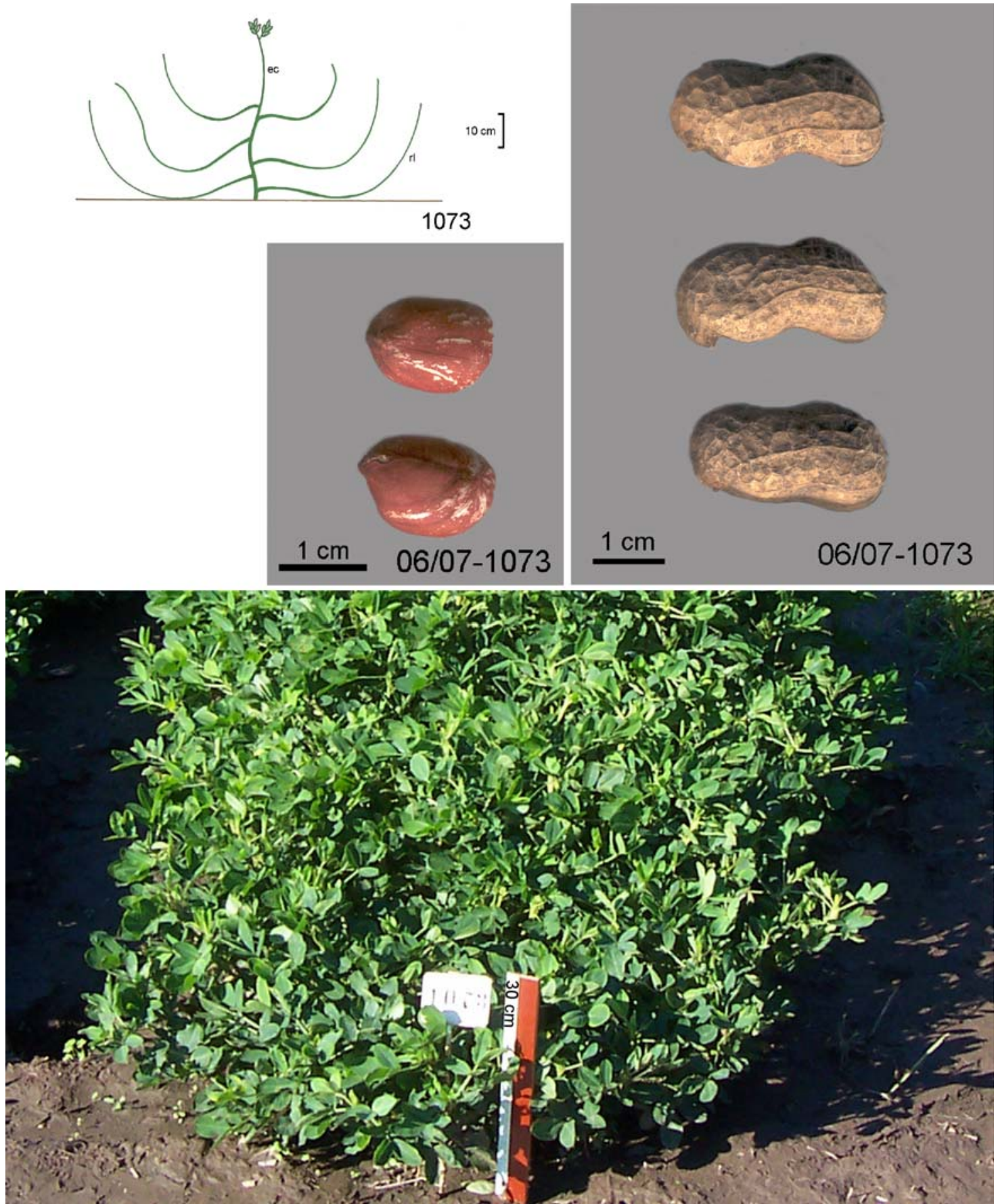


\section{Overo}

Planta erecta, ramas rectas (se ven los clavos).

Cajas con poca carena, algo estranguladas, 2 granos, colorado fuerte y albo.

$\begin{array}{llll}\text { 06/07 } & 1077 & 85 / 862148 & \text { US } 342 \text { KSBScCo } \\ 1078 & 85 / 862239 & \text { US } 753 \text { GKP }\end{array}$

Potosi, Villazón

Sta. Cruz, Santa Cruz
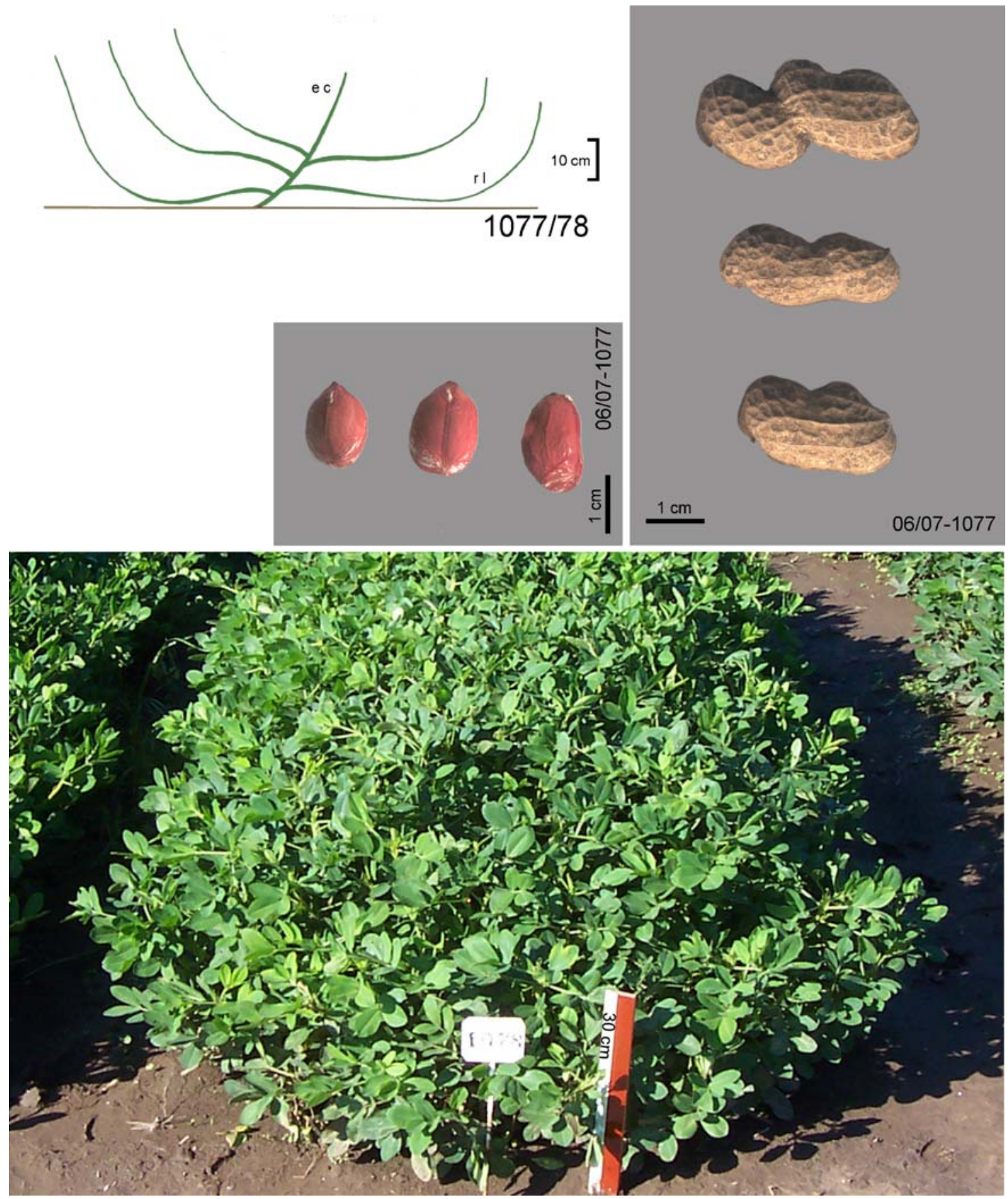
Krapovickas, A.\& al., Las razas de maní de Bolivia

\begin{tabular}{|c|c|c|c|c|}
\hline $85 / 862236$ & $\mathrm{RCM}$ & 1452 & K & Cochabamba, Cochabamba \\
\hline 85/86 2237 & " & 1458 & $\mathrm{~K}$ & ", $\quad$ ", \\
\hline 85/86 2238 & " & 1465 & $\mathrm{~K}$ & Sta. Cruz, Samaipata \\
\hline 85/86 2241 & $"$ & 1471 & KMoF & Tarija, San Telmo \\
\hline 85/86 2242 & $"$ & 1476 & " & Tarija, San Simón \\
\hline 85/86 2243 & $"$ & 1478 & " & Tarija, Yacuiba \\
\hline $85 / 862244$ & " & 1486 & " & Tarija, Hualcalques \\
\hline 85/86 2245 & " & 1488 & " & Tarija, Entre Ríos \\
\hline 85/86 2248-49 & $"$ & 30 & $"$ & " \\
\hline 85/86 2262 & " & 34 & " & Sta. Cruz, Boyuibe \\
\hline $85 / 862263$ & " & 36 & " & Sta. Cruz, Lagunillas \\
\hline $85 / 862265$ & " & 35 & " & " \\
\hline 85/86 2273 & " & 36 & " & " \\
\hline 85/86 2277 & " & 37 & " & Sta. Cruz, Gutierrez \\
\hline 85/86 2278 & " & 37 & " & " \\
\hline 85/86 2287 & " & 39 & & Sta. Cruz, EEA Saavedra \\
\hline $85 / 862290$ & " & 40 & " & $\begin{array}{llll}\prime \prime & \prime & \prime & \end{array}$ \\
\hline 85/86 2294 & " & 41 & " & $\begin{array}{llll}\prime \prime & \prime & \prime & m\end{array}$ \\
\hline 85/86 2296 & " & 43 & GKSBPSc & Sta. Cruz, Santa Cruz, mercado \\
\hline 85/86 2297 & " & 46 & " & " " " " \\
\hline 85/86 2301 & " & 48 & BPZ & Sta. Cruz, Yerba Buena \\
\hline 85/86 2303 & " & 61 & " & Sta. Cruz, Trigal, Vallegrande \\
\hline 85/86 2306 & " & 95 & " & Cochabamba, Cochabamba \\
\hline 85/86 2309 & " & 97 & " & " \\
\hline 85/86 2311 & " & 100 & " & La Paz, La Paz \\
\hline 85/86 2314 & " & 101 & " & " $\quad$ " \\
\hline 85/86 2318 & $"$ & 101 & " & " m \\
\hline 85/86 2321 & " & 118 & GKSSc & Sta. Cruz, San Ignacio \\
\hline 85/86 2329 & " & 202 & GKSPScGb & \\
\hline 85/86 2331 & " & 204 & ", & Sta. Cruz, Santa Cruz, mercado \\
\hline 85/86 2332 & $"$ & 208 & $"$ & \\
\hline 85/86 2333 & $"$ & 213 & GKSPSc & Beni, Trinidad, \\
\hline 85/86 2335 & " & 219 & ", & Beni, San Borja \\
\hline 85/86 2336 & " & 301 & KSBScCo & Potosí, Villazón \\
\hline 85/86 2337 & $"$ & 302 & ", & " " " \\
\hline 85/86 2338 & " & 303 & " & ", \\
\hline 85/86 2340 & $"$ & 305 & " & " \\
\hline 85/86 2341 & " & 307 & " & " \\
\hline 85/86 2342 & " & 308 & " & " \\
\hline 85/86 2343 & " & 313 & " & " \\
\hline 85/86 2345 & $"$ & 320 & " & " \\
\hline 85/86 2346 & " & 323 & " & " \\
\hline 85/86 2347 & " & 325 & " & Sta Cruz, Santa Cruz, mercado \\
\hline 85/86 2348 & " & 328 & " & " " \\
\hline 85/86 2349 & US & 330 & " & " \\
\hline 85/86 2350 & " & 340 & " & ", \\
\hline 85/86 2393 & " & 342 & KSBScCo & Sta. Cruz, Santa Cruz \\
\hline 85/86 2351 & $"$ & 345 & "' & " \\
\hline 85/86 2352 & $"$ & 352 & BZCo & La Paz, La Paz, mercado \\
\hline 85/86 2353 & " & 353 & ", & " \\
\hline 85/86 2354 & " & 354 & " & " \\
\hline
\end{tabular}




\begin{tabular}{|c|c|c|c|c|c|}
\hline 85/86 2355 & US & 358 & \multicolumn{2}{|c|}{ BZCo } & La Paz, La Paz, mercado \\
\hline 85/86 2356 & " & 373 & \multicolumn{2}{|c|}{ ", } & \\
\hline 85/86 2357 & " & 384 & \multicolumn{2}{|c|}{ BZCoJ } & La Paz, N. Yungas Coroico \\
\hline 85/86 2358 & " & 399 & & Sta. Cruz, San Matías \\
\hline 85/86 2359 & " & 400 & \multicolumn{2}{|c|}{$\begin{array}{c}\text { KSSC } \\
\text { ", }\end{array}$} & Sta. Cruz, Santiago \\
\hline $85 / 862360$ & " & 402 & \multicolumn{2}{|c|}{ " } & Sta. Cruz, Natividad \\
\hline 85/86 2361 & " & 619 & \multicolumn{2}{|l|}{$\mathrm{PZi}$} & Chuquisaca, Sucre, mercado \\
\hline $85 / 862240$ & " & 752 & \multicolumn{2}{|c|}{ GKP } & Sta. Cruz, Roboré \\
\hline 85/86 2362 & " & \multirow{2}{*}{\multicolumn{3}{|c|}{$\begin{array}{l}\text { 807-2 KSSc } \\
819-1\end{array}$}} & Tarija,Tarija, mercado \\
\hline 85/86 2363 & " & & & & rarija, 1 arija, illercado \\
\hline 85/86 2364 & " & \multicolumn{3}{|c|}{ 823-1 KSScCr } & Tarija, Entre Ríos \\
\hline 85/86 2365 & $"$ & \multicolumn{3}{|c|}{ 824-1 "' } & " " " " \\
\hline 85/86 2366 & " & \multicolumn{3}{|l|}{$826-1$} & " \\
\hline 85/86 2367 & ", & \multicolumn{3}{|l|}{$828-1$} & "' \\
\hline 85/86 2368 & " & \multicolumn{3}{|c|}{$829-1$} & " \\
\hline 85/86 2386 & " & \multicolumn{3}{|c|}{ 829-2 KSScCo } & Tarija, San Simón \\
\hline 85/86 2369 & $"$ & \multicolumn{3}{|c|}{ 830-1 " } & Tarija, San Simón \\
\hline 85/86 2370 & " & $831-1$ & & " & ", " " \\
\hline 85/86 2371 & $"$ & $832-3$ & & " & Tarija, Entre Ríos \\
\hline 85/86 2372 & " & 833-1 & & " & $\begin{array}{lll}\prime \prime & \prime\end{array}$ \\
\hline 85/86 2373 & ", & 835-1 & & " & ', \\
\hline 85/86 2374 & $"$ & $837-1$ & & " & " \\
\hline 85/86 2375 & " & 840-1 & KSS & & Tarija, Villa Montes \\
\hline 85/86 2376 & " & $841-1$ & & & " " " \\
\hline 85/86 2377 & " & 844-1 & & & " \\
\hline 85/86 2378 & " & $852-1$ & & & Tarija, Yacuiba \\
\hline 85/86 2379 & $"$ & 853-1 & & & " \\
\hline 85/86 2380 & " & $855-2$ & " & & Tarija, Villa Montes \\
\hline 89/90 1980 & & 698 & $\mathrm{Wi}$ & Pintado & Sta Cruz, Yotaú-Ñ. Chavez \\
\hline 89/90 1981 & & 700 & Wi & guarayo & Urubichá, "' "' \\
\hline 89/90 1982 & & 703 & $\mathrm{Wi}$ & manduv & arayo) " " " \\
\hline 89/90 1983 & & 710 & $\mathrm{Wi}$ & maní & Beni, San Ignacio de Moxos \\
\hline 89/90 1984 & & 742 & $\mathrm{Wi}$ & maní & Beni, Rurrenabaque \\
\hline 89/90 1985 & & 743 & $\mathrm{Wi}$ & overo & " " " \\
\hline 89/90 1986 & & 870 & $\mathrm{Wi}$ & maní & Beni, Trinidad \\
\hline
\end{tabular}




\section{Overo carenado}

Planta erecta , ramas erectas, se ven los clavos.

Cajas con retículo marcado, redondeada, con carena y algo de pico, con 2, (3) granos, colorado y albo.
06/07 1079
Potosí, Villazón
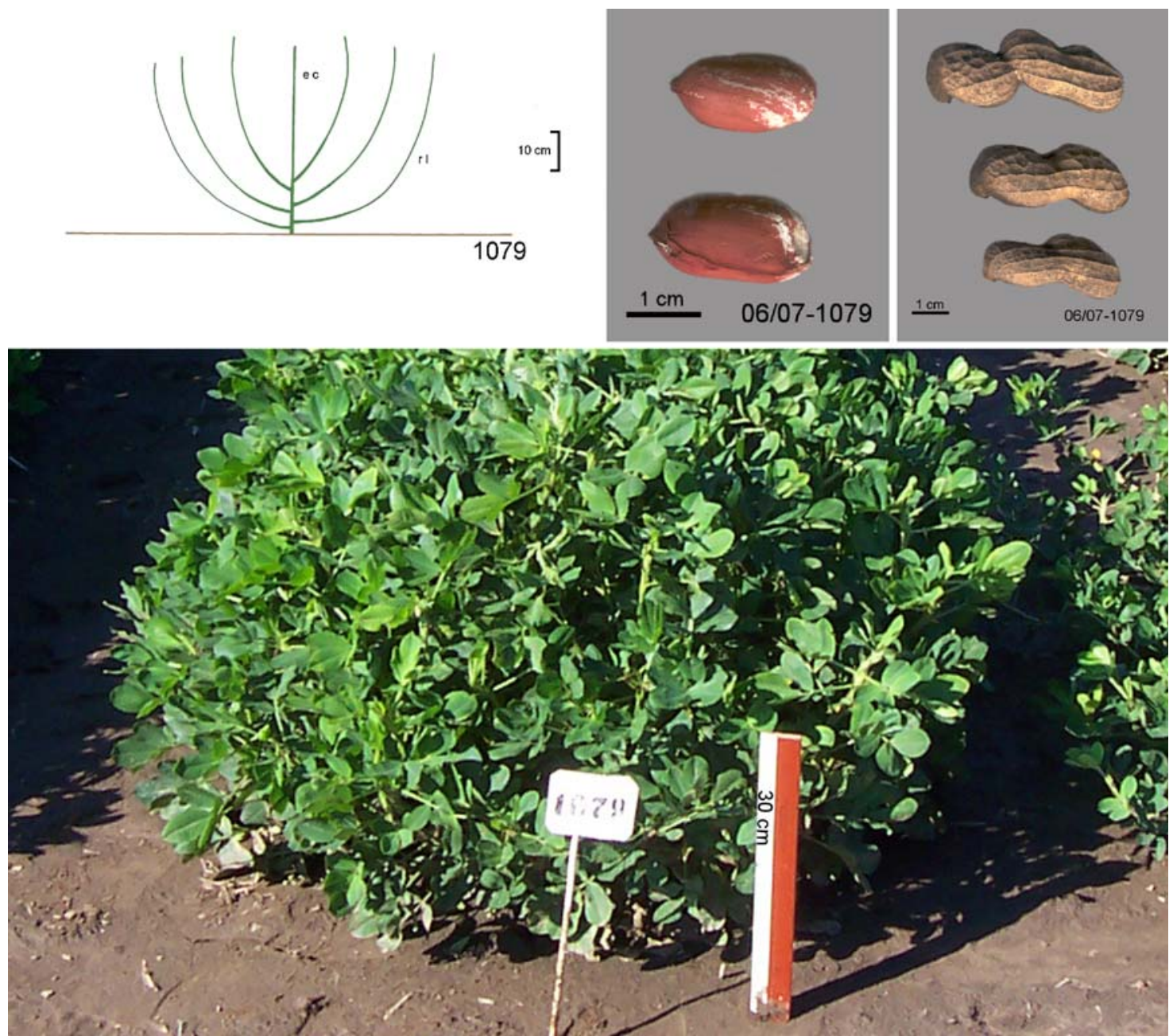

$85 / 862233$

$85 / 862234$

$85 / 862235$

$85 / 862205$

85/86 2208

85/86 2211-1

$85 / 862212$

85/86 2212

85/86 2199

85/86 2227

$$
\begin{aligned}
& \text { RCM } 1445 \text { K } \\
& \text { " } 1446 \mathrm{~N} \\
& \text { " } 1447 \mathrm{~N} \\
& \text { " 315-2 KSBScCo } \\
& \text { " } \quad 356 \text { BZCo } \\
& \text { " } 371 \\
& \text { " } 390 \text { BZCoJ } \\
& \text { " } 390 \\
& \text { US } \quad 616 \quad \mathrm{PZi} \\
& \text { " 840-1 KSSc }
\end{aligned}
$$

Cochabamba, Cochabamba Sta. Cruz, Mairana

$$
\text { " " " }
$$

Potosí, Villazón

La Paz, La Paz, mercado

La Paz, N Yungas Caranavi

Chuquisaca, Sucre, mercado Tarija, Villa Montes 


\section{Colorado precoz}

Planta con tallo verde, apenas morado. Ramas dísticas o en algunas apenas torcidas en el ápice.

Cajas chicas, poco reticuladas, con carena, muchas de 3 granos colorados.

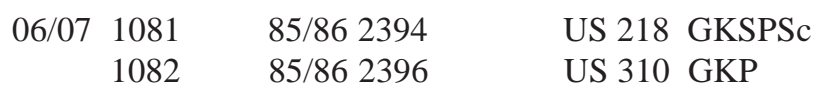

1082
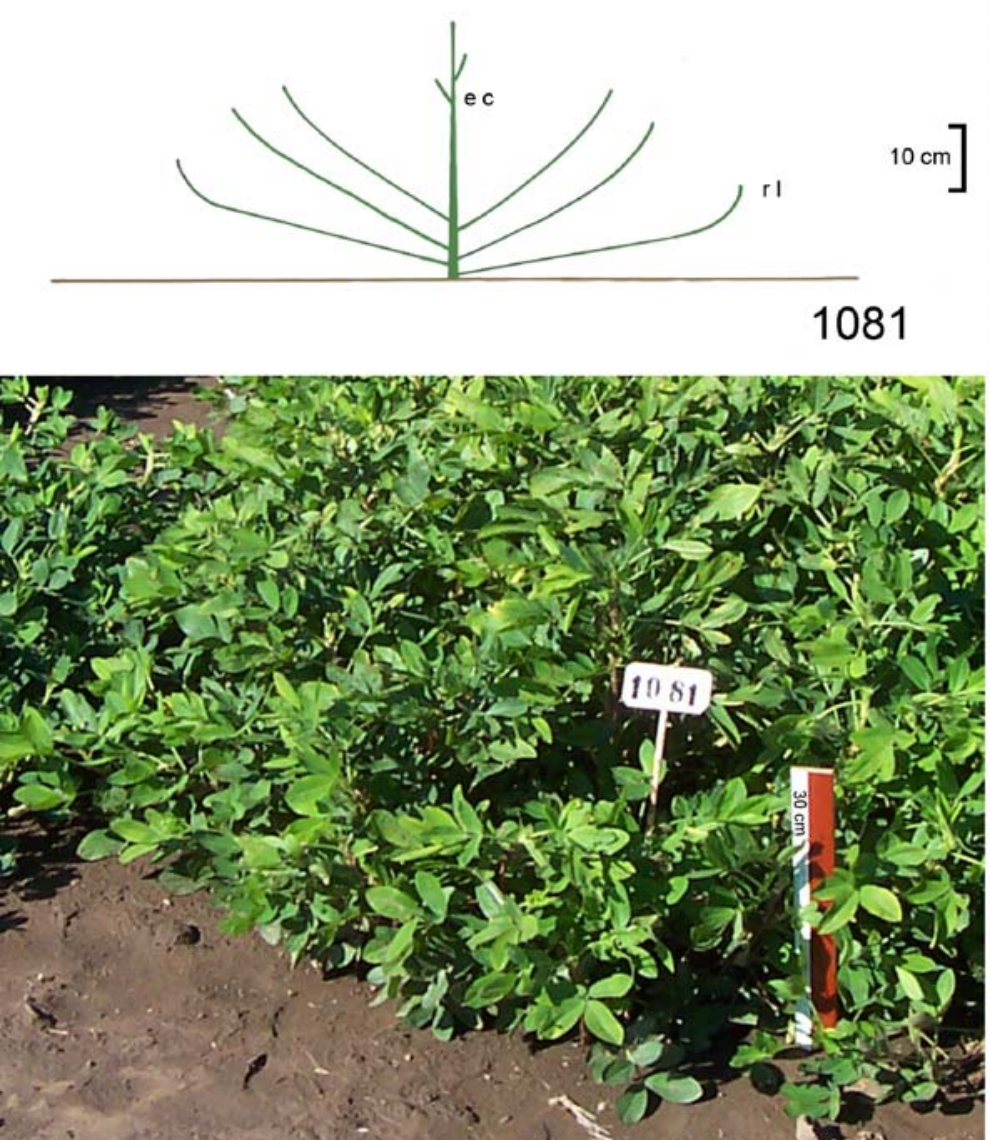

$85 / 862407$

85/86 2409

$85 / 862411$

$85 / 862412$

$85 / 862416$

85/86 2417

$85 / 862420$

$85 / 862397$

$85 / 862399$

$85 / 862400$

$85 / 862402$

$85 / 862403$

85/86 2404
US 44

" 203

" 203

" 205

" 218

" 218

" 218

" 326

" 327

" 329

" 341

" 372

" 375
GKBSPSc colorado chico

GKSPScGb maní chico

GKSPSc

',

KSBScCo

".

BZCo colorado
Beni, San Borja

Sta. Cruz, EE Los Llanos

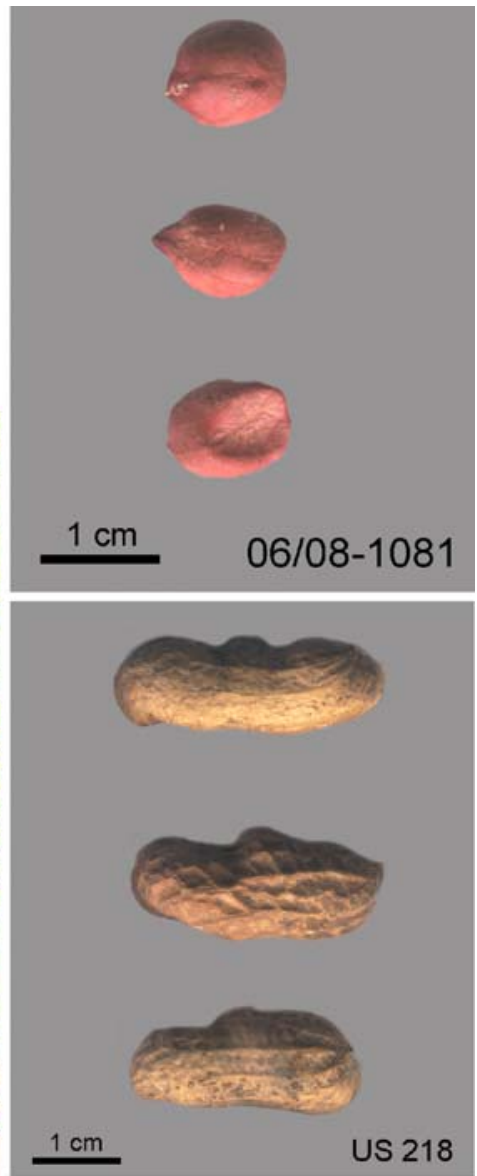

Sta Cruz, Sta. Cruz

", ", ",

", " "

Beni, S. Borja

", ",

Sta. Cruz, Santa Cruz

". ".

La Paz, La Paz 


\section{Colorado distico de Entre Rios}

Planta tipo Valencia, muy erecto, poco ramificada. 1/2. UNICO.

Cajas chicas, redondeadas, algo estranguladas, con 2 (3), granos, colorado fuerte.
06/07 1103
85/86 2529
US 828-2 KSSc
Tarija, Entre Ríos
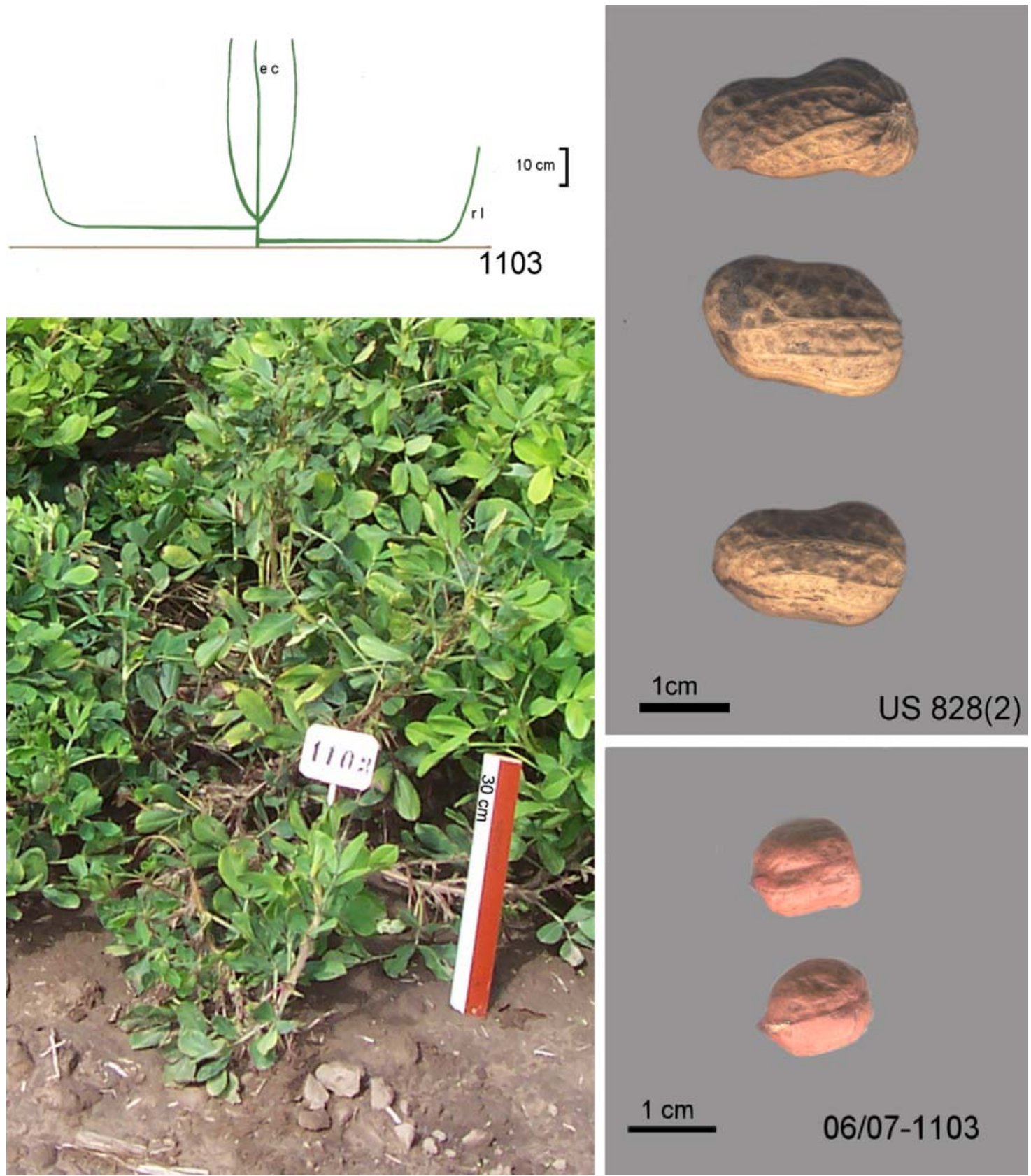


\section{Colorado Menonitas}

Planta precoz (15-III-90 sin hojas). Tallo verde, apenas morado, 2/5.

Cajas con joroba y pico, 4 granos colorados.

Material posiblemente introducido por los Menonitas de México.
06/07 1085
$85 / 862480$
US $401 \mathrm{KSSc}$
Sta. Cruz, S. José Chiquitos
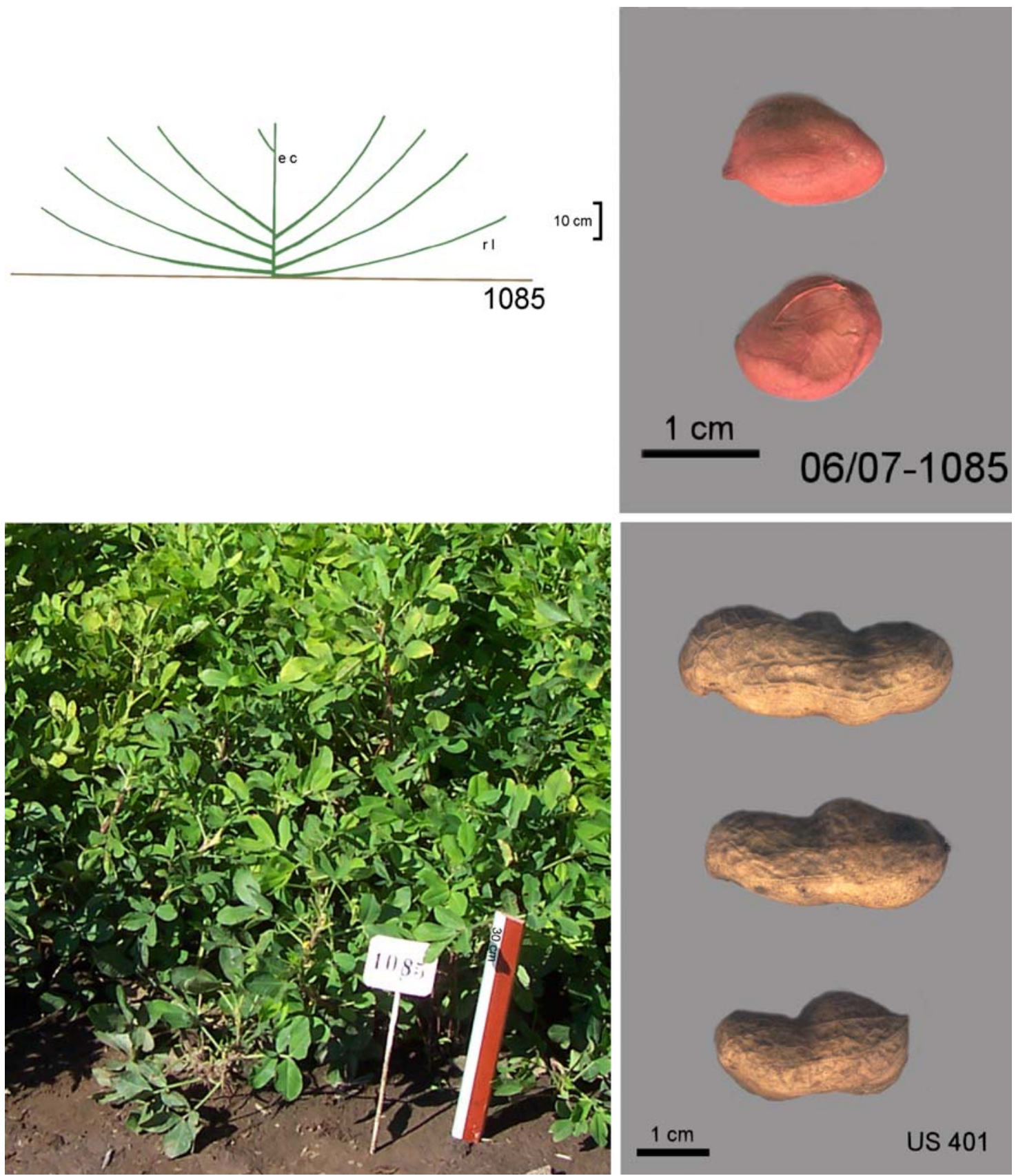


\section{Colorado precoz compacto}

Planta compacta, verde oscuro, precoz. Es más erecto que el Colorado precoz, ramas 2/5.

Cajas poco reticuladas, las pequeñas redondeadas, las grandes con joroba y carena; 3-4 granos colorado sucio.
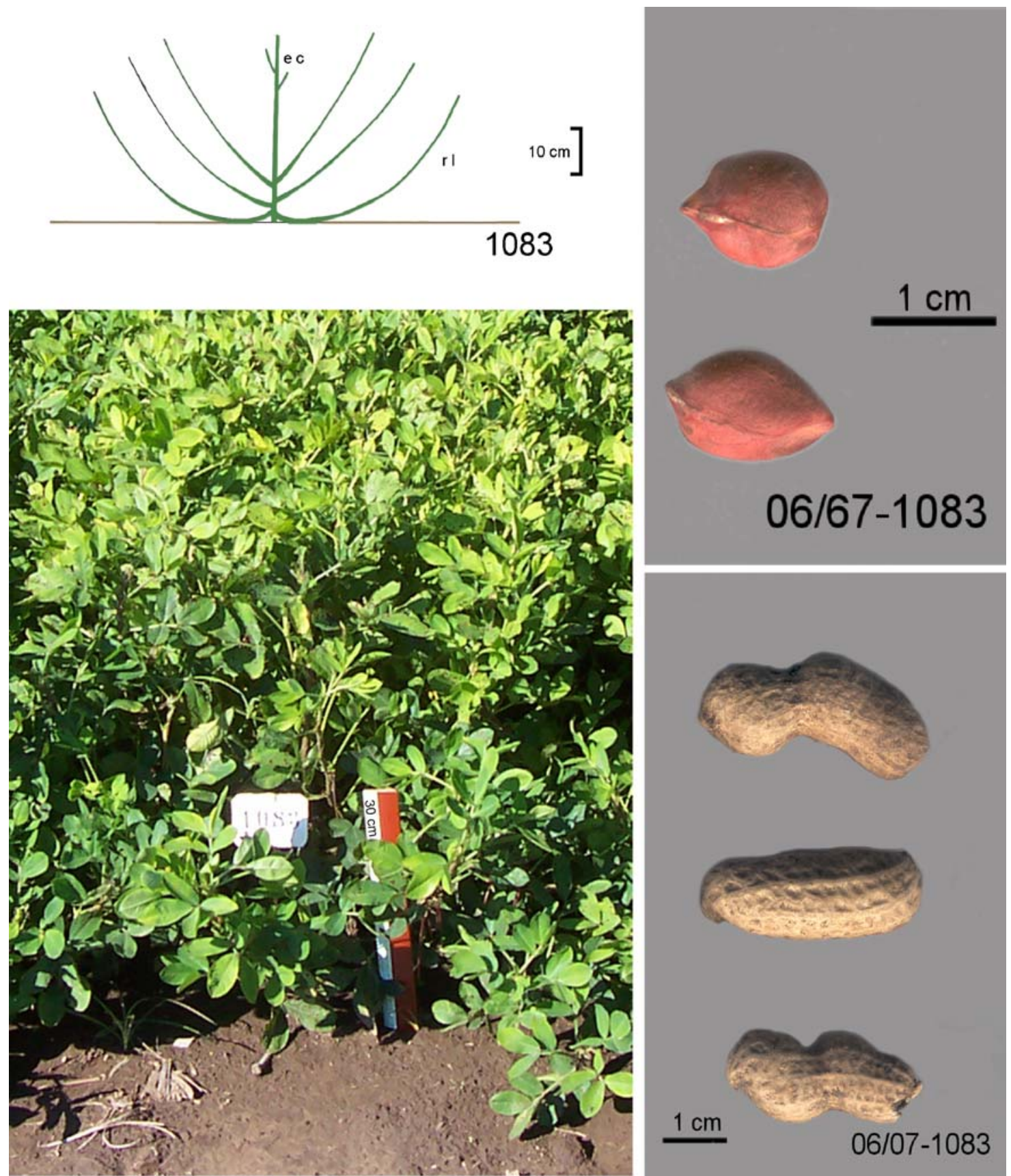


\section{Precoz de Villa Montes}

Planta erecta. Eje central más ramificado. Tallo verde, apenas morado, 2/5. Precoz. Cajas poco reticuladas, redondeadas 2-3 granos colorados.
06/07 1084
85/86 2447
US 845-1 KSSc
Tarija, Villa Montes
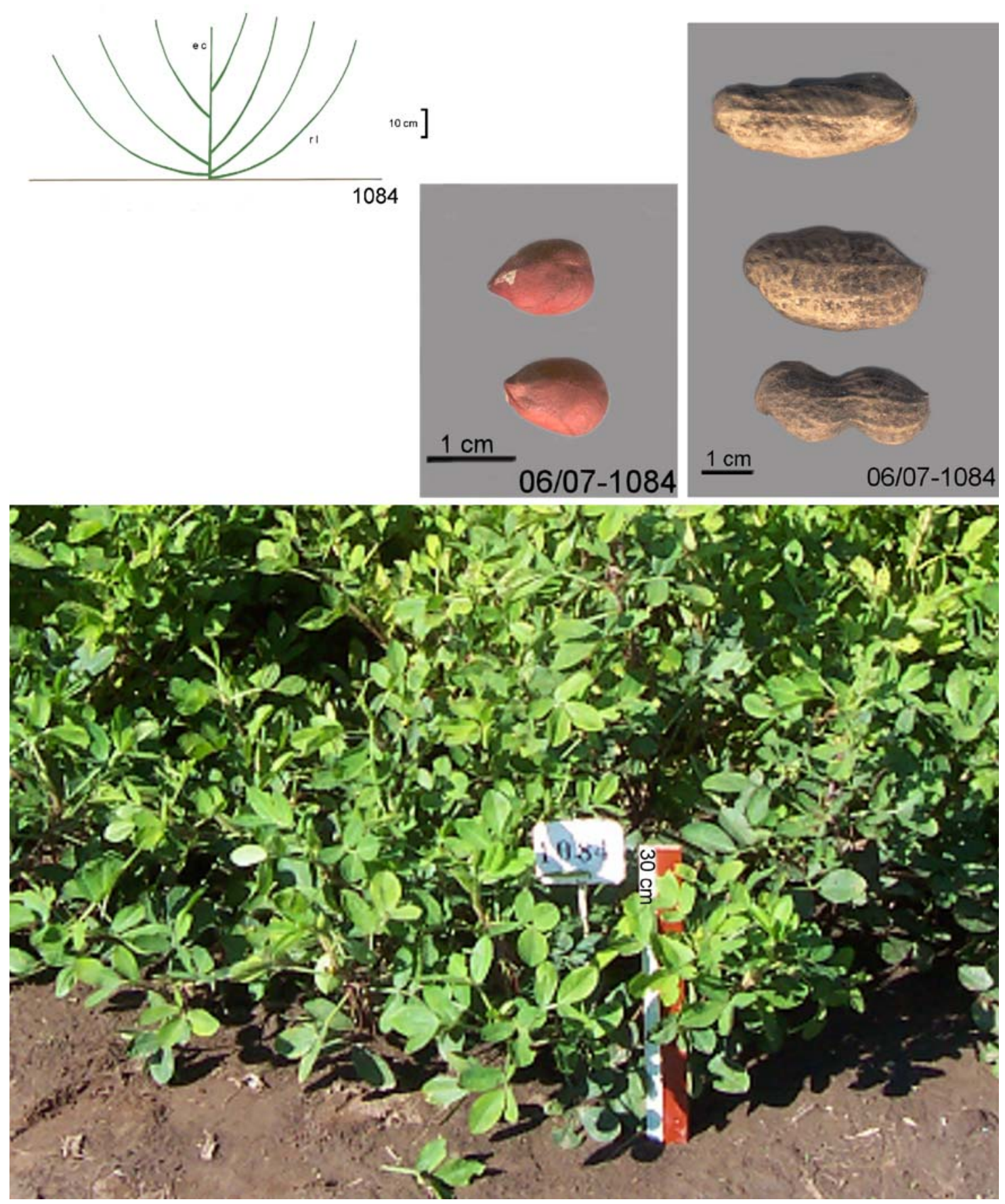


\section{Cartucho}

Planta erecta, tallos verdes, folíolos grandes, muy desarrollados.

Cajas con 3-4 granos, con poca constricción y retículo grueso, diluído. Granos colorados.

$\begin{array}{rlll}06 / 071086 & 85 / 862422 & \text { RCM 1477 KMoF } & \text { Tarija, Villa Montes } \\ 1087 & 85 / 862423 & \text { RCM 1479 KMoF } & \text { Tarija, Yacuiba }\end{array}$
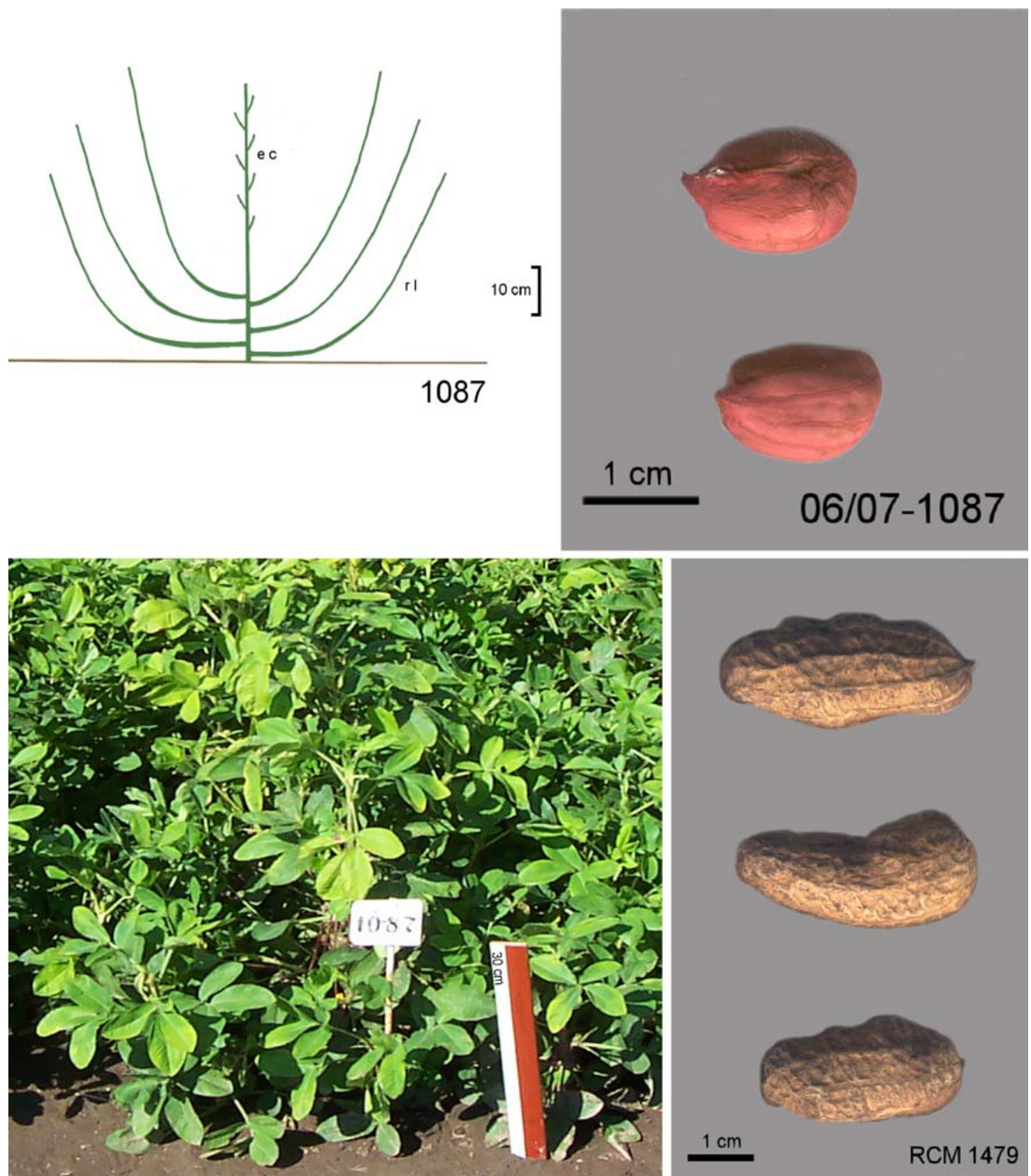
BONPLANDIA 18(2). 2009

\begin{tabular}{|c|c|c|c|c|c|}
\hline $85 / 862424$ & US & 16 & GKBSPSc & Cdo. Del palmar & Tarija, Aguayrenda \\
\hline 85/86 2425 & $"$ & 19 & " & Cartucho & Tarija, Carapari \\
\hline 85/86 2426 & $"$ & 24 & $"$ & & Tarija, Villa Montes \\
\hline 85/86 2430 & " & 26 & " & & ", \\
\hline 85/86 2431 & " & 28 & " & Cartucho & Tarija, Villa Montes \\
\hline 85/86 2432 & ' & 28 & $"$ & & \\
\hline 85/86 2432-2 & "' & 28 & " & & \\
\hline 85/86 2433 & " & 32 & $"$ & & Tarija, V. Montes \\
\hline 85/86 2437 & " & 33 & " & Cototo & Tarija, Villa Montes \\
\hline 85/86 2440 & " & 812 & KSSc & & Tarija,Tarija \\
\hline 85/86 2441 & $"$ & 822 & " & & Tarija, Entre Ríos \\
\hline 85/86 2442 & $"$ & 830-1 & $"$ & & Tarija, Sebingal "' \\
\hline 85/86 2443 & " & 838 & $"$ & & Tarija, P. Margarita \\
\hline 85/86 2444 & $"$ & 839-1 & " & & Tarija, V. Montes \\
\hline $85 / 862445$ & " & 842 & ' & & " " " \\
\hline 85/86 2446 & $"$ & 843-1 & $"$ & & $"$ \\
\hline $85 / 862448$ & $"$ & 846-1 & $"$ & & " \\
\hline 85/86 2449 & " & $847-1$ & "' & & " \\
\hline $85 / 862450$ & "' & 848 & " & & " \\
\hline 85/86 2451 & "' & 849 & " & & " \\
\hline 85/86 2452 & "' & 850 & " & & " \\
\hline $85 / 862453$ & $"$ & 854-1 & $"$ & & " \\
\hline
\end{tabular}




\section{Colorado de Cero Ocho}

Planta poco ramificada (ca. 5 ramas) 2/5, algo moradas. Ramas decumbentes, con casi todas reproductivas y 1 a 2 vegetativas en el ápice. Precoz (con pocas hojas al 15-3-91). Con cerdas.

Cajas lisas 3- 4 granos, colorado sucio.

\section{6/07 1093 89/90 2013 US 721-1 Wi Cero ocho \\ Beni, Yacuma, Jaibat yabá (Chimane) 1094 89/90 2014 US 723 Wi maní colorado cero ocho Cahuátke yabá (Chimane)}
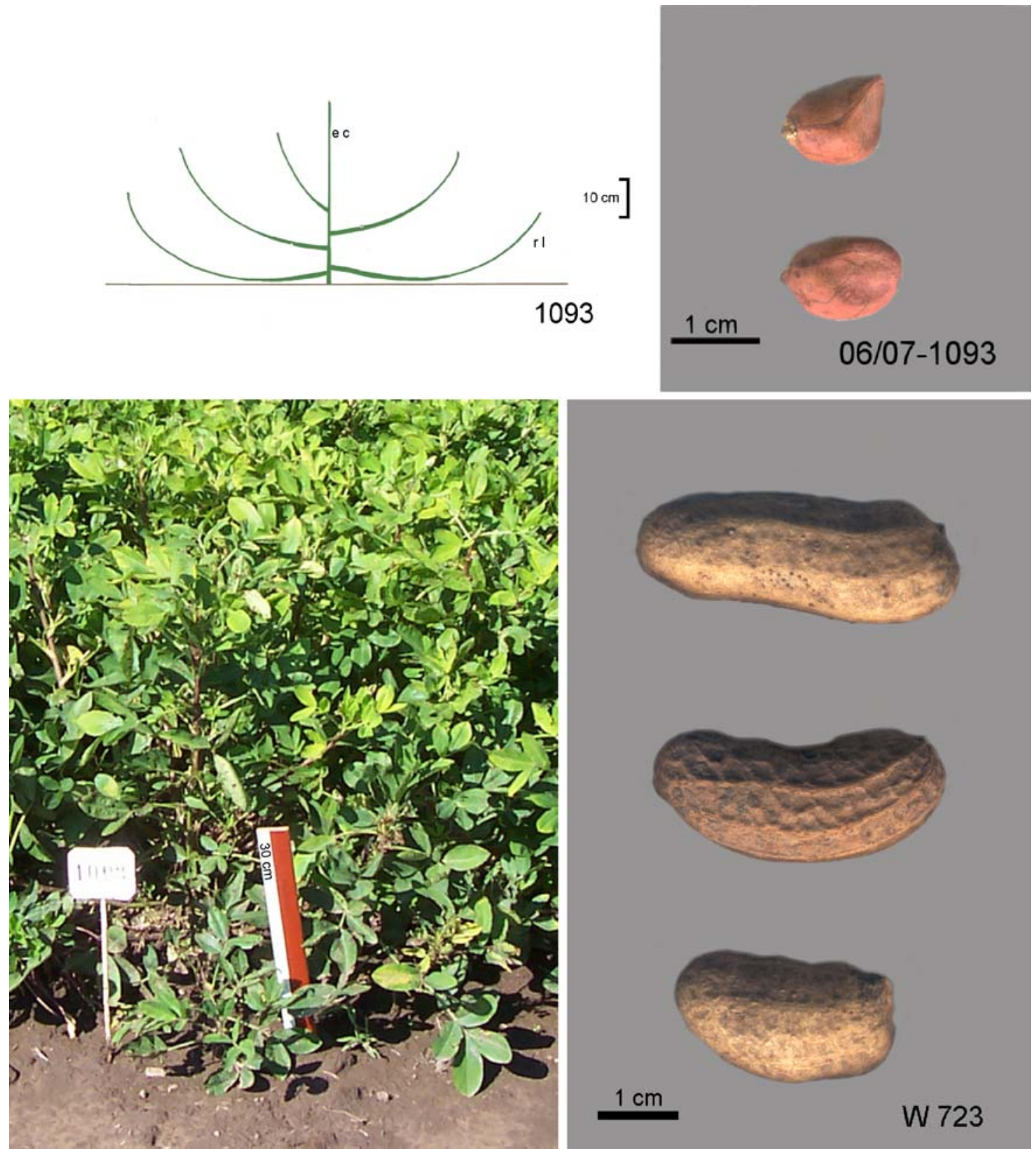


\section{Cruceño}

Planta decumbentes. Tallos verdes con algo de tinte violáceo, sin cerdas, a veces con algunas de ellas. Eje central muy ramificado.

Cajas con retículo marcado, grueso, 3-4 granos, colorados.

$\begin{array}{rrrrr}06 / 07 & 1088 & 85 / 862459 & \text { RCM 1455 K } & \text { Cochabamba, Cochabamba } \\ 1089 & 85 / 862460 & \text { RCM 1459 K } & \text { Sta. Cruz, Santa Cruz. }\end{array}$
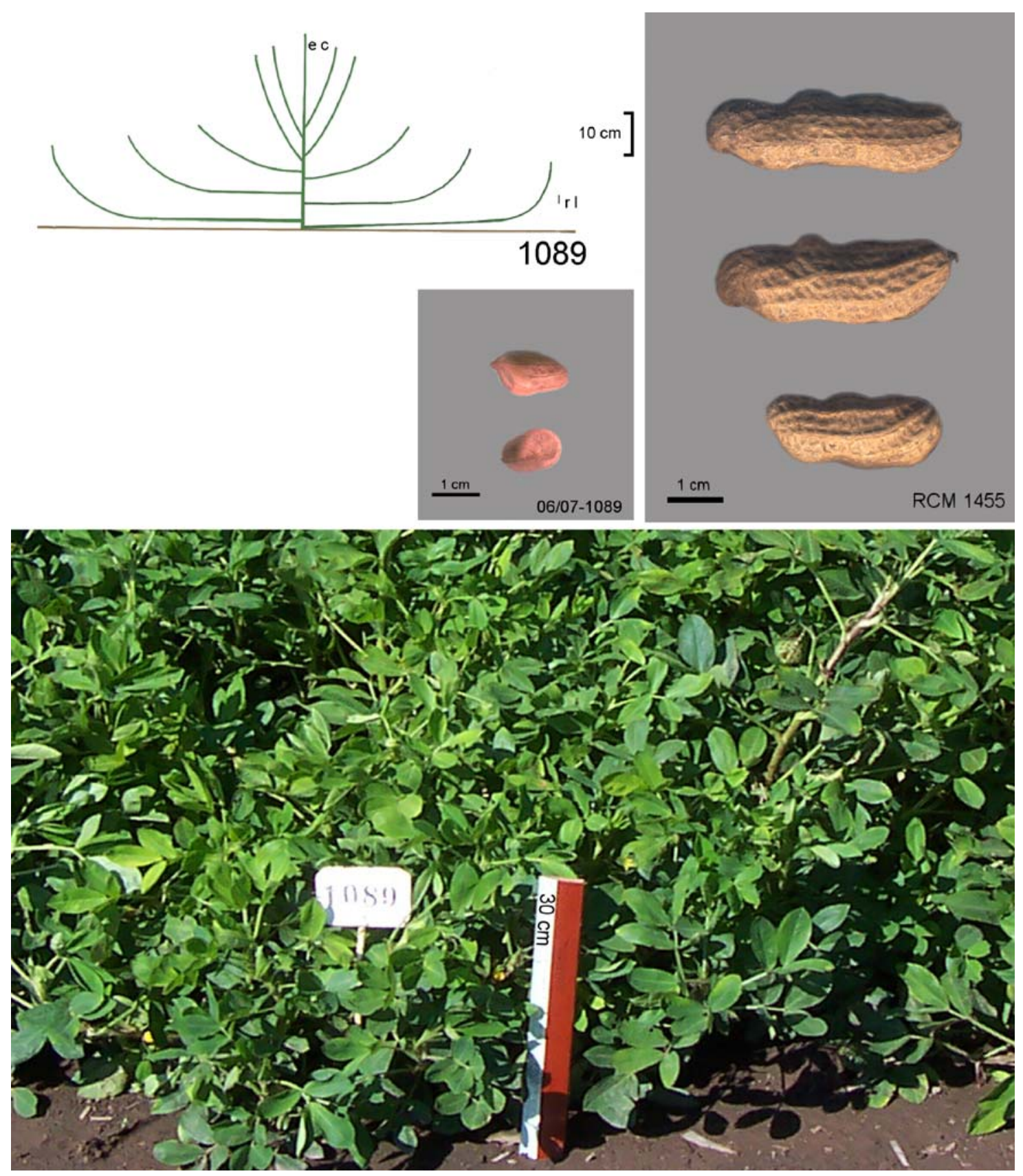
Krapovickas, A.\& al., Las razas de maní de Bolivia

\begin{tabular}{|c|c|c|c|}
\hline 85/86 2461 & \multicolumn{2}{|c|}{ RCM 1460} & $\mathrm{~K}$ \\
\hline 85/86 2462 & US & 45 & GKBSPSc \\
\hline 85/86 2463 & " & 45 & \\
\hline 85/86 2474 & $"$ & 46 & GKBSPSc \\
\hline 85/86 2466 & $"$ & 72 & $\mathrm{BPZ}$ \\
\hline 85/86 2469 & " & 93 & \\
\hline $85 / 862454$ & US & 201 & GKSPScGb \\
\hline 85/86 2455 & " & 212 & \\
\hline 85/86 2456 & $"$ & 216 & GKSPSc \\
\hline 85/86 2475 & $"$ & 217 & GKSPSc \\
\hline $85 / 862481$ & $"$ & 286 & GKP \\
\hline 85/86 2482 & $"$ & 287 & $"$ \\
\hline 85/86 2483 & $"$ & 288 & $"$ \\
\hline 85/86 2484 & $"$ & 289 & $"$ \\
\hline 85/86 2485 & $"$ & 290 & $"$ \\
\hline 85/86 2486 & $"$ & 291 & $"$ \\
\hline 85/86 2487 & " & 292 & $"$ \\
\hline 85/86 2488 & $"$ & 293 & $"$ \\
\hline 85/86 2489 & $"$ & 294 & " \\
\hline 85/86 2490 & $"$ & 295 & " \\
\hline 85/86 2491 & $"$ & 296 & $"$ \\
\hline 85/86 2492 & $"$ & 297 & " \\
\hline 85/86 2493 & $"$ & 298 & $"$ \\
\hline 85/86 2494 & $"$ & 299 & " \\
\hline 85/86 2496 & " & 301 & " \\
\hline 85/86 2476 & $"$ & 327 & KSBScCo \\
\hline 85/86 2477 & $"$ & 331 & " \\
\hline 85/86 2478 & $"$ & 333 & $"$ \\
\hline 85/86 2479 & $"$ & 339 & " \\
\hline 89/90 2209 & " & 925 & Wi \\
\hline
\end{tabular}

Sta. Cruz, Santa Cruz

Sta. Cruz, Santa Cruz

Sta. Cruz, Mairana

Cochab., Cochabamba

Sta. Cruz, Sta. Cruz

Sta. Cruz, EEA Saavedra

Beni, Magdalena

Beni, Huacaraje

Sta. Cruz, Warnes

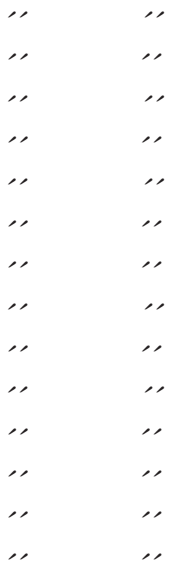

Sta. Cruz, Santa Cruz

" " " "

" $"$ " , ,

", " , " 


\section{Colorado de Rurrenabaque}

Planta grande, ramas sinuosas, gruesas, verdes, con algo de tinte violáceo, 2/5. Hojas grandes. Cajas casi lisas, poco reticuladas, con algo de carena, sin pico. 3-4 granos, colorado sucio.
$\begin{array}{rr}06 / 07 & 1090 \\ 1091\end{array}$
$85 / 862520$
US $604-2$ PZi
US 606
Beni, San Buenaventura
Beni, Rurrenabaque

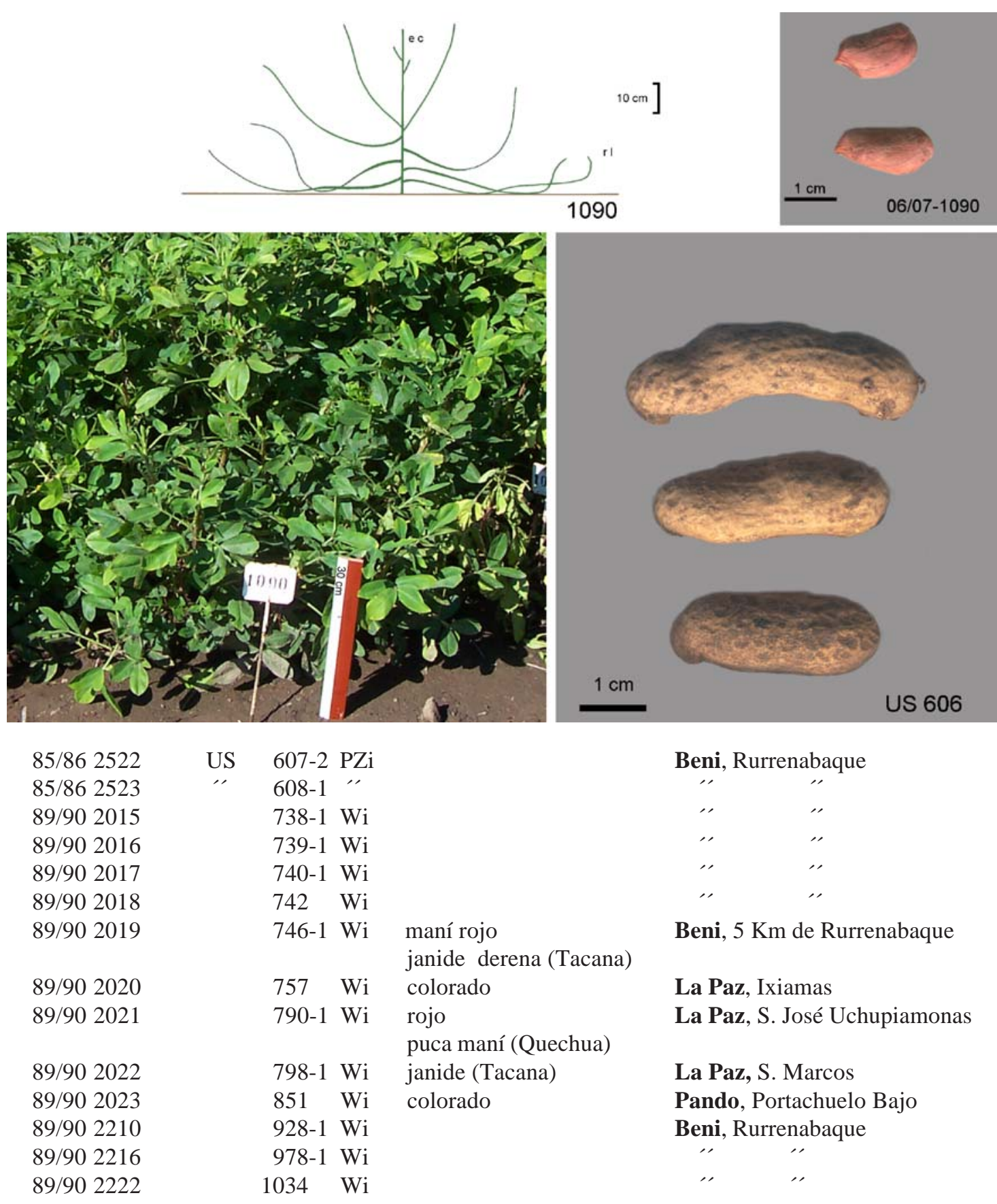




\section{Colorado picudo}

Planta erecta, muy abierta, eje central muy aparente. Con cerdas.

Cajas algo más reticuladas que el Colorado de Rurrenabaque, algo de joroba, con carena y pico breve. 3-4 granos colorados.
06/07 1092
$85 / 862525$
US 377 BZCo
La Paz, La Paz
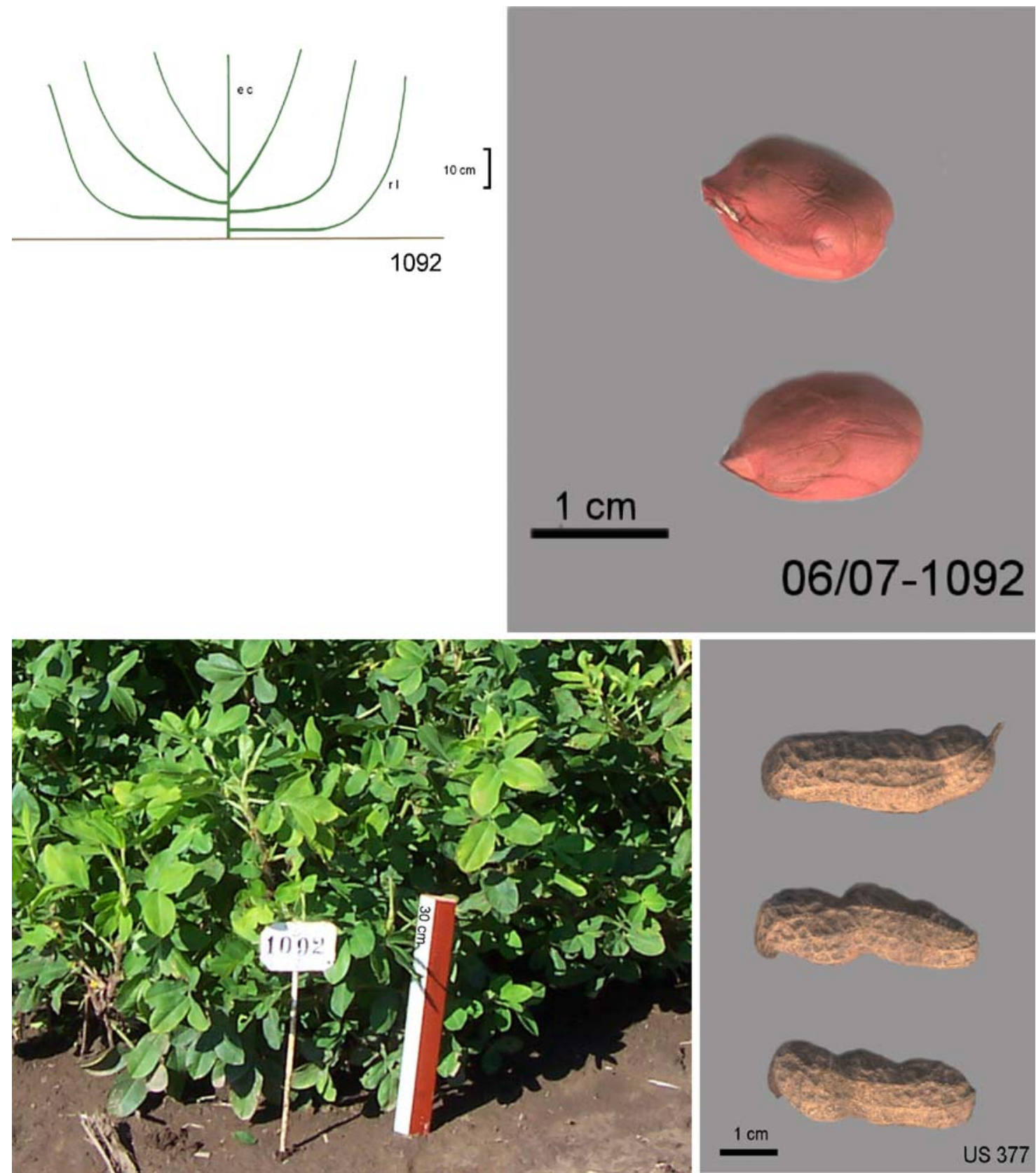


\section{Colorado cáscara negra}

Planta ramificado, pequeña.

Cajas con cáscara negra, con jorobas y carena, 4 granos colorado.

\begin{tabular}{|c|c|c|c|}
\hline 06/07 1095 & $89 / 902219$ & 1010 Wi cáscara negra & Beni, Rurrenabaque \\
\hline 1096 & 90/91 2627 & $1143 \mathrm{Wi}$ ". " & Beni, Rurrenabaque \\
\hline
\end{tabular}
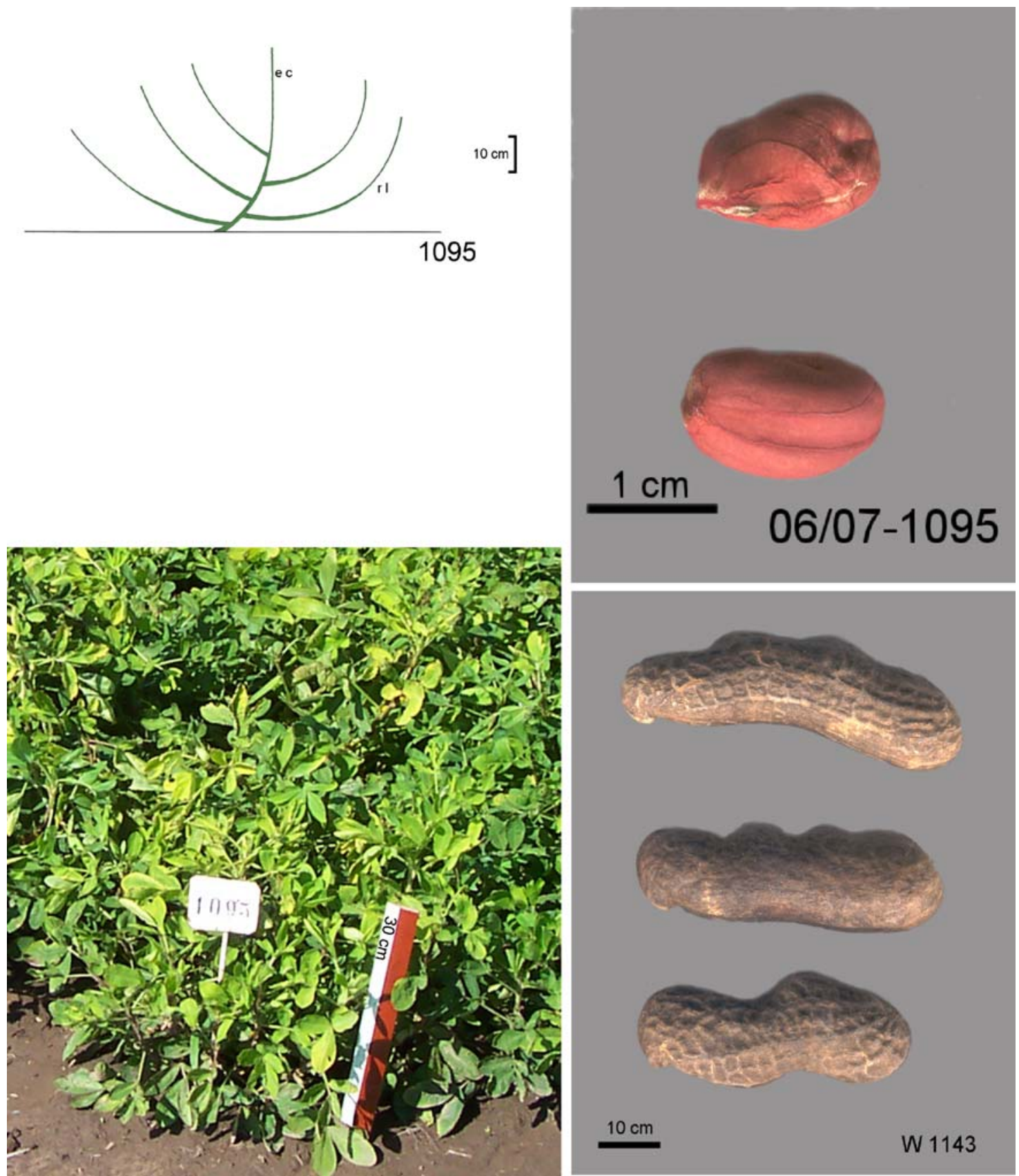


\section{Pálido del Beni}

Planta grande (algo más pequeña que el Colorado de Rurrenabaque). Tallo grueso, verde, algo morado, muy ramificado, 2/5. Eje central algo aparente. Hojas grandes. Clavos morados. Sin cerdas o con 1-2 cerdas en las estípulas.

Cajas grandes, casi lisas, retículo grueso, con jorobas, con carena y poco pico, 3-4 granos pálidos, casi albos.

$\begin{array}{rr}06 / 071097 & 85 / 862505 \\ 1098 & 85 / 862508\end{array}$

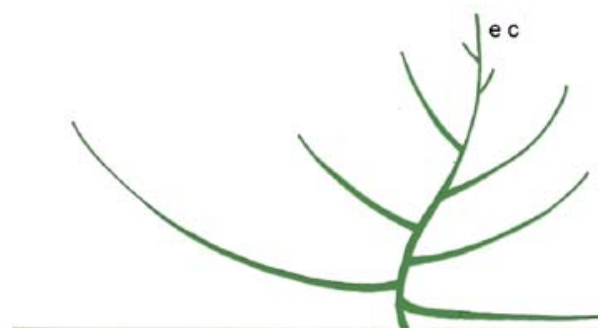

US $106 \mathrm{BPZ}$

US 225 GKSPSc

La Paz, S. Antonio Chulumani

Beni, Riberalta
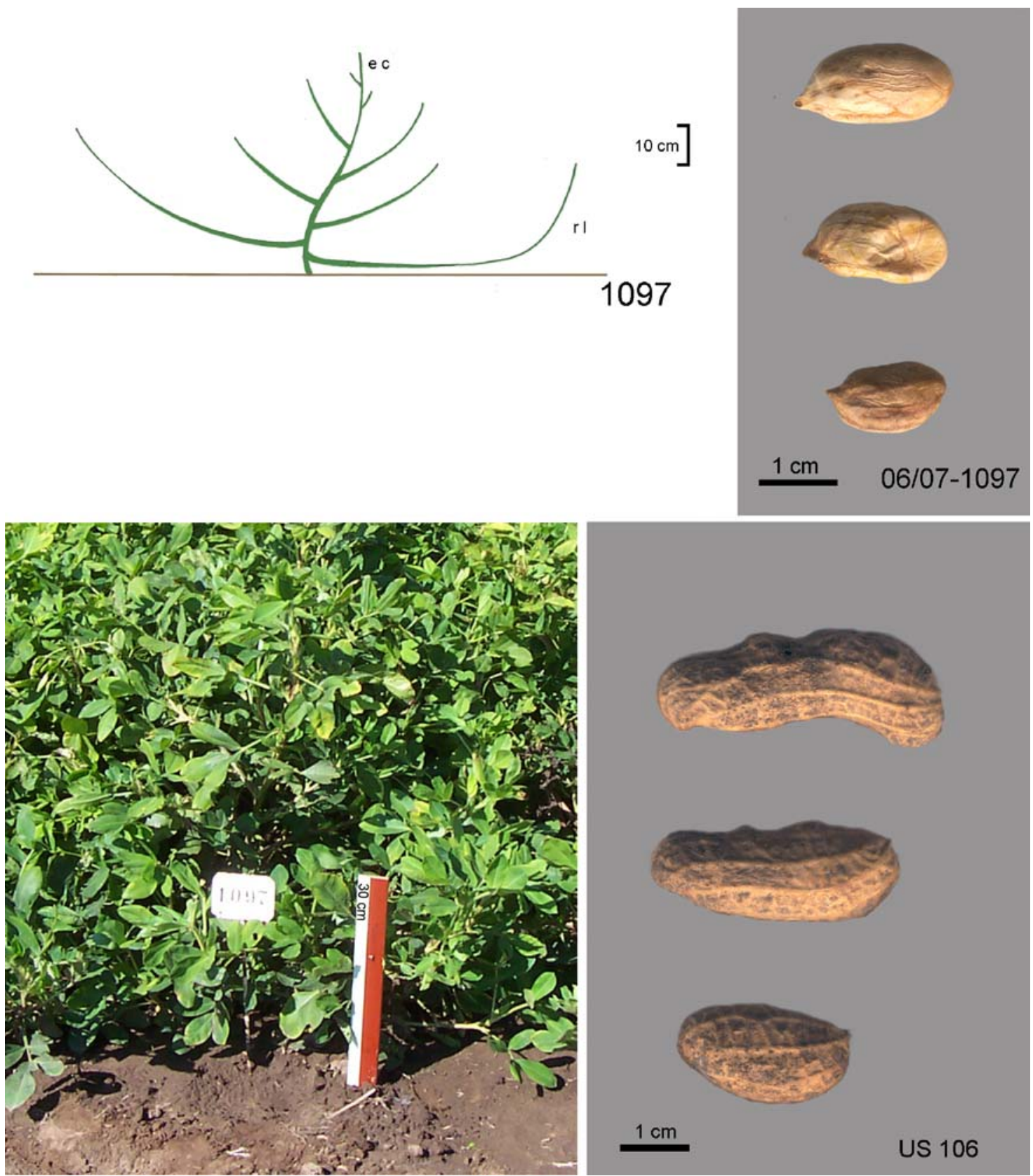

1097

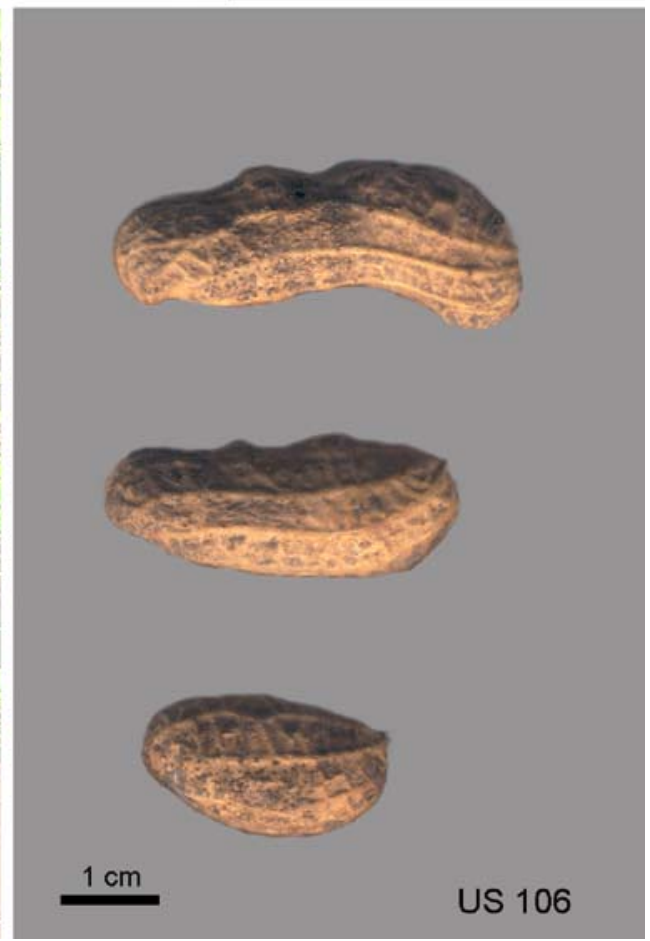




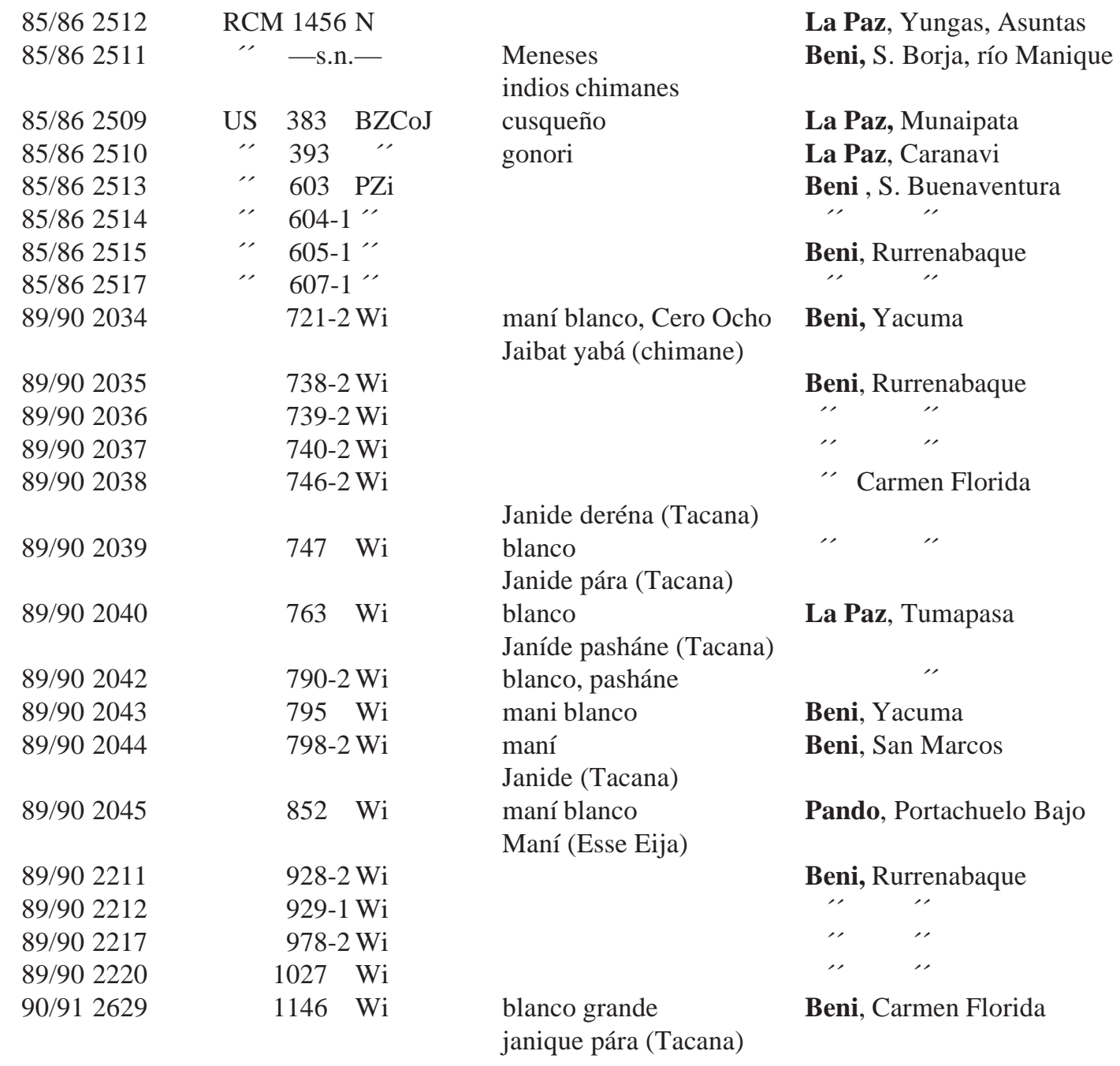




\section{Pálido de Uchupiamonas}

Planta grande. Hojas grandes. Sin cerdas. Clavos o verdes.

Cajas con retículo grueso, poco marcado, algo de jorobas, sin pico ni carena; 3-4 granos pálido.
06/07 1099
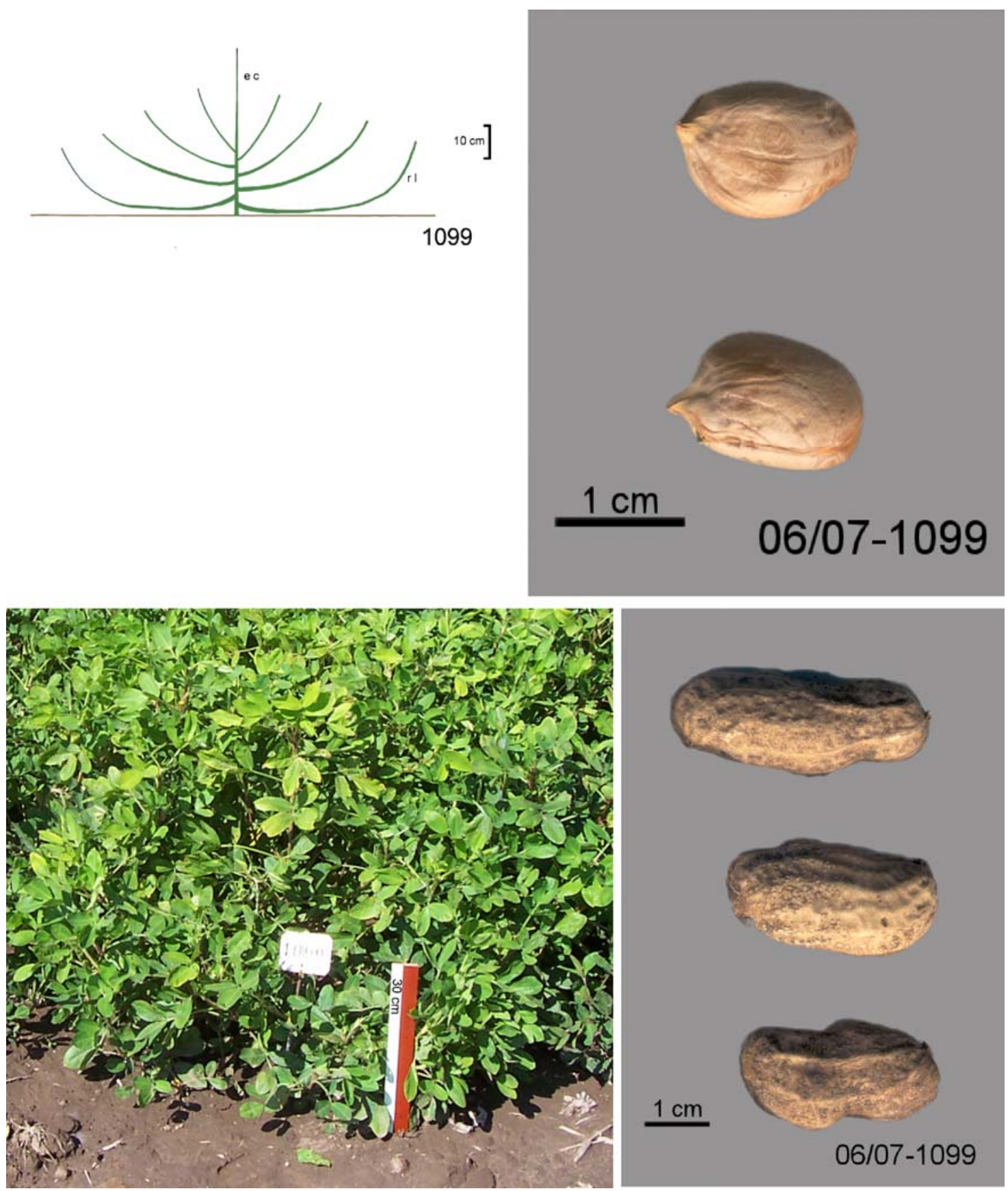


\section{Negro de Rurrenabaque}

Planta grande, poco ramificada, ramas con 0-1 vegetativas, semierectas y caídas; apenas torcidas en el ápice. Tallo violáceo; brote violáceo; con pocas cerdas.

Cajas algo reticuladas, retículo grueso. Algo de jorobas, con algo de carena y pico. 4 granos negros.

$\begin{array}{llllll}06 / 07 & 1100 & 85 / 862518 & \text { US 607 } & \text { PZi } & \text { Beni, Rurrenabaque } \\ 1101 & 89 / 902213-1 & \text { US 929-2 } & \text { Wi } & \text { Beni, Rurrenabaque }\end{array}$
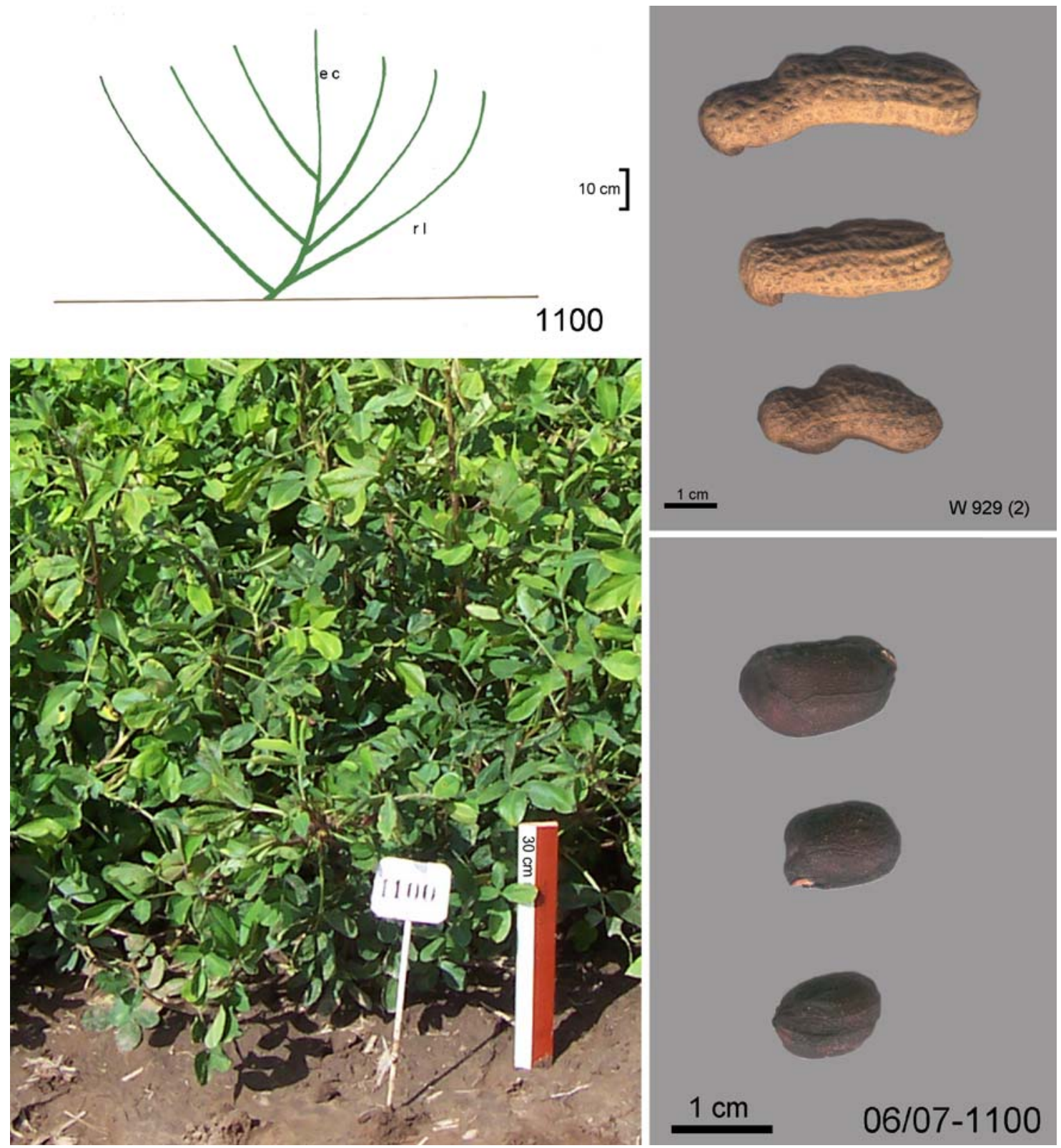

$89 / 902214 \quad 930$

$\mathrm{Wi}$

Beni, Rurrenabaque 


\section{Rosado pálido}

Planta tipo valencia, pequeña, baja erecta, poco ramificada. Eje central apenas aparente, 2/ 5.Tallo verde, algo morado, clavos morados. UNICO.

Cajas chicas, redondeadas, algo estranguladas, con 2 (3) granos pálidos, algo rosados.
06/07 1102
85/86 2528-1
US 847-2 KSSc
Tarija, Villa Montes
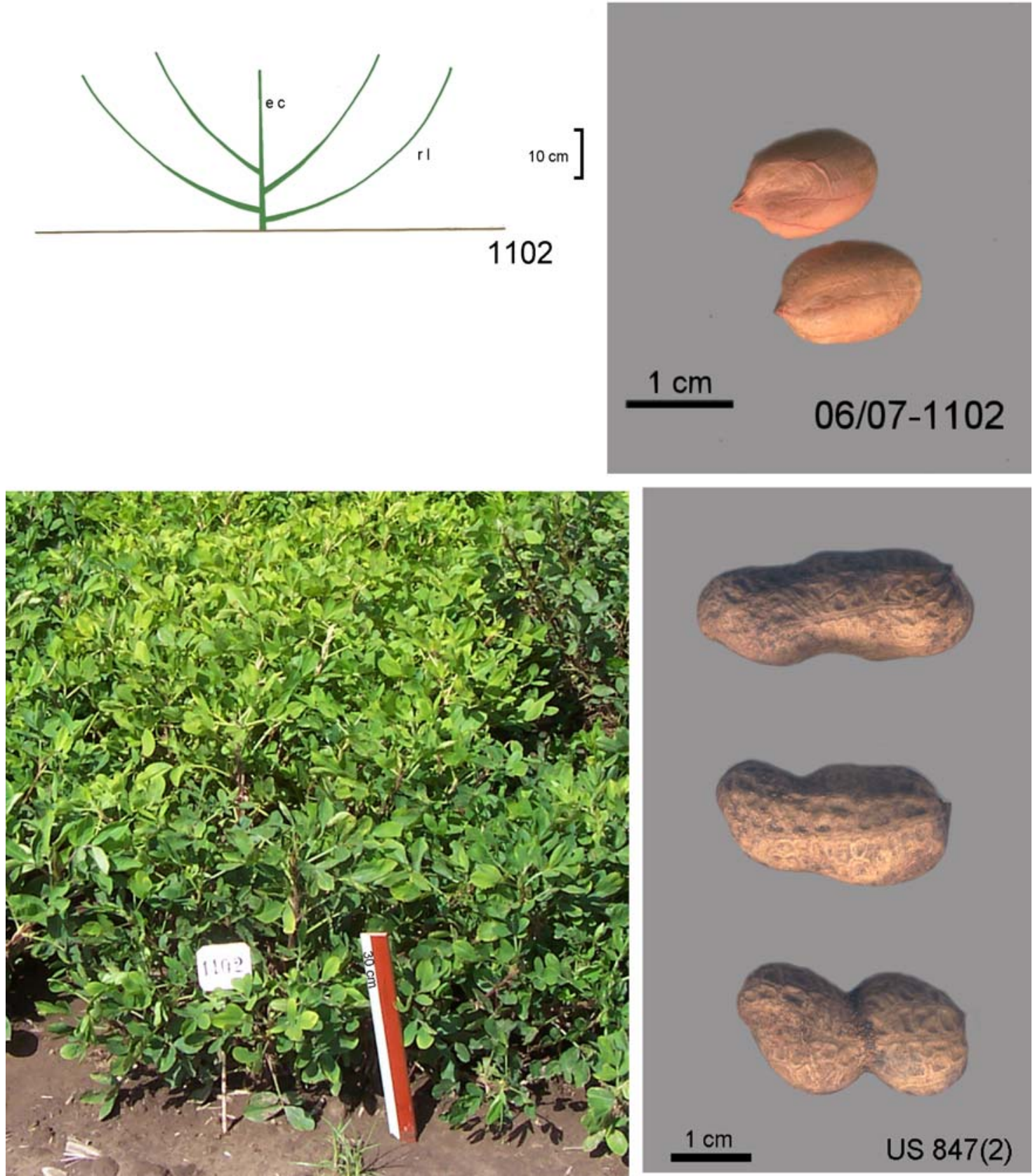


\section{Amarillo}

Planta poco ramificada, ramas erectas y pocas, caídas. 2/5 Tallo verde. Clavo verde. Sin cerdas. Hojas grandes.

Cajas casi lisas, con retículo grueso. Algo de jorobas; con carena. 4 granos.
06/07 1104.
$89 / 902048766 \mathrm{Wi}$
89/90 $22211028 \mathrm{Wi}$
amarillo
La Paz, río Tuíche Beni, Rurrenabaque

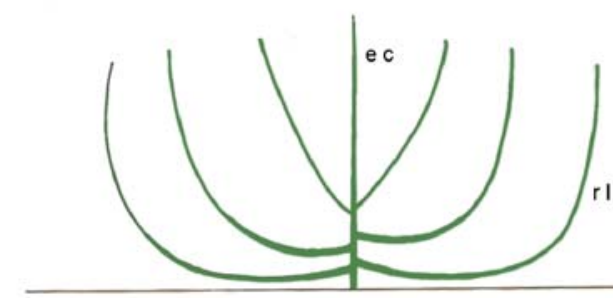

1104
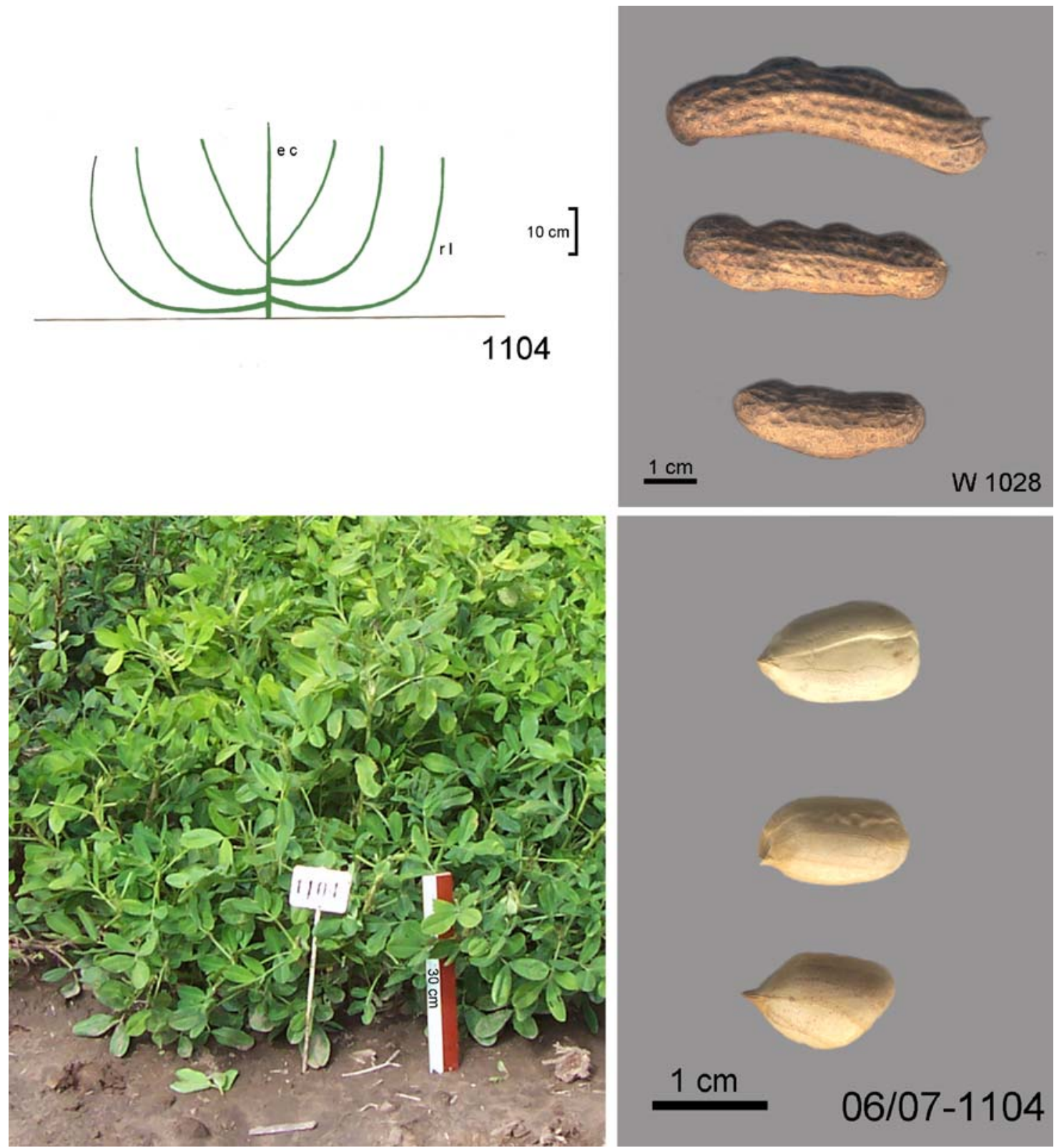

$90 / 912628$
$1144 \mathrm{Wi}$

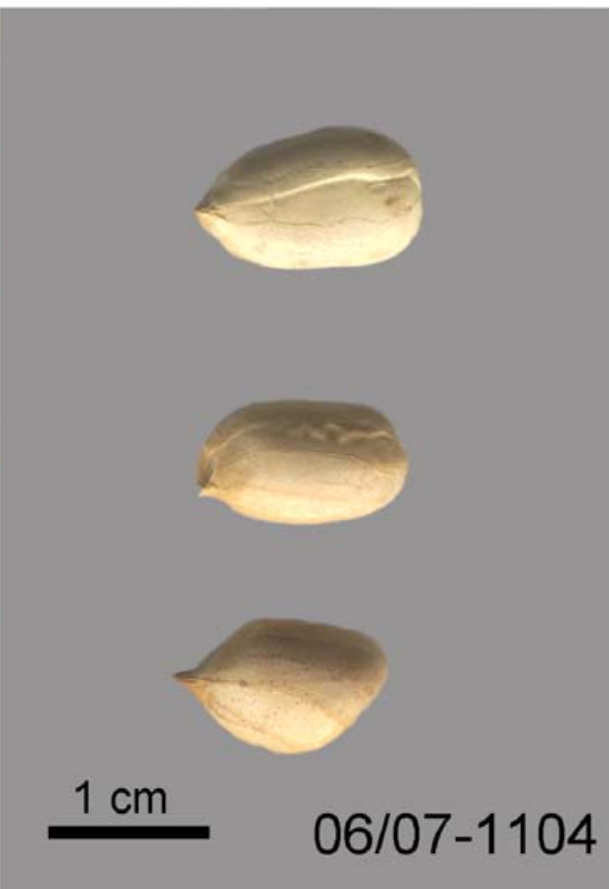

Beni, Carmen Florida amarillo Janide (Tacana) 


\section{Albo}

Planta chica poco ramificada, ramas erectas (Tallos hasta $30 \mathrm{~cm}$ long.) $2 / 5$ y 1/2. Tallos verde, clavo verde. Estandarte sin aro.

Cajas parecidas al "amarillo", pero más pequeñas. Granos albo verdosos.
06/07 1106
$85 / 862519$
US $607 \mathrm{PZi}$
Beni, Rurrenabaque
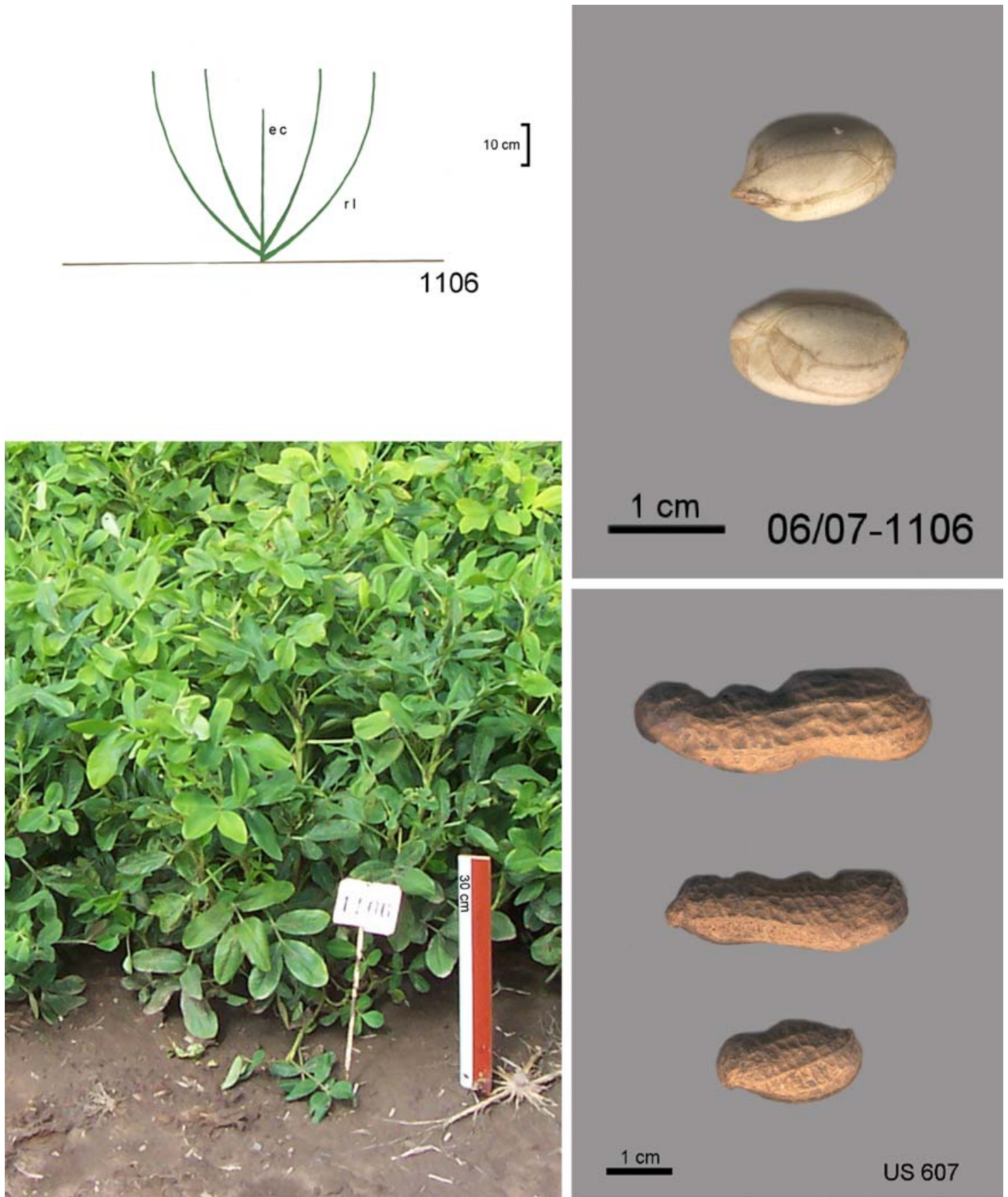


\section{Palido de Villa Montes}

Planta tipo Spanish, pequeña, compacta. Eje central no aparente, 1/2.

Cajas chicas, redondeadas, algo estranguladas. 2(3) granos pálidos.

$\begin{array}{llll}06 / 07 & 1107 & 85 / 862528-2 & \text { US 847-2 KSSc } \\ 1108 & 85 / 862531 & \text { US 854-2 KSSc }\end{array}$

Tarija, Villa Montes

Tarija, Yacuiba
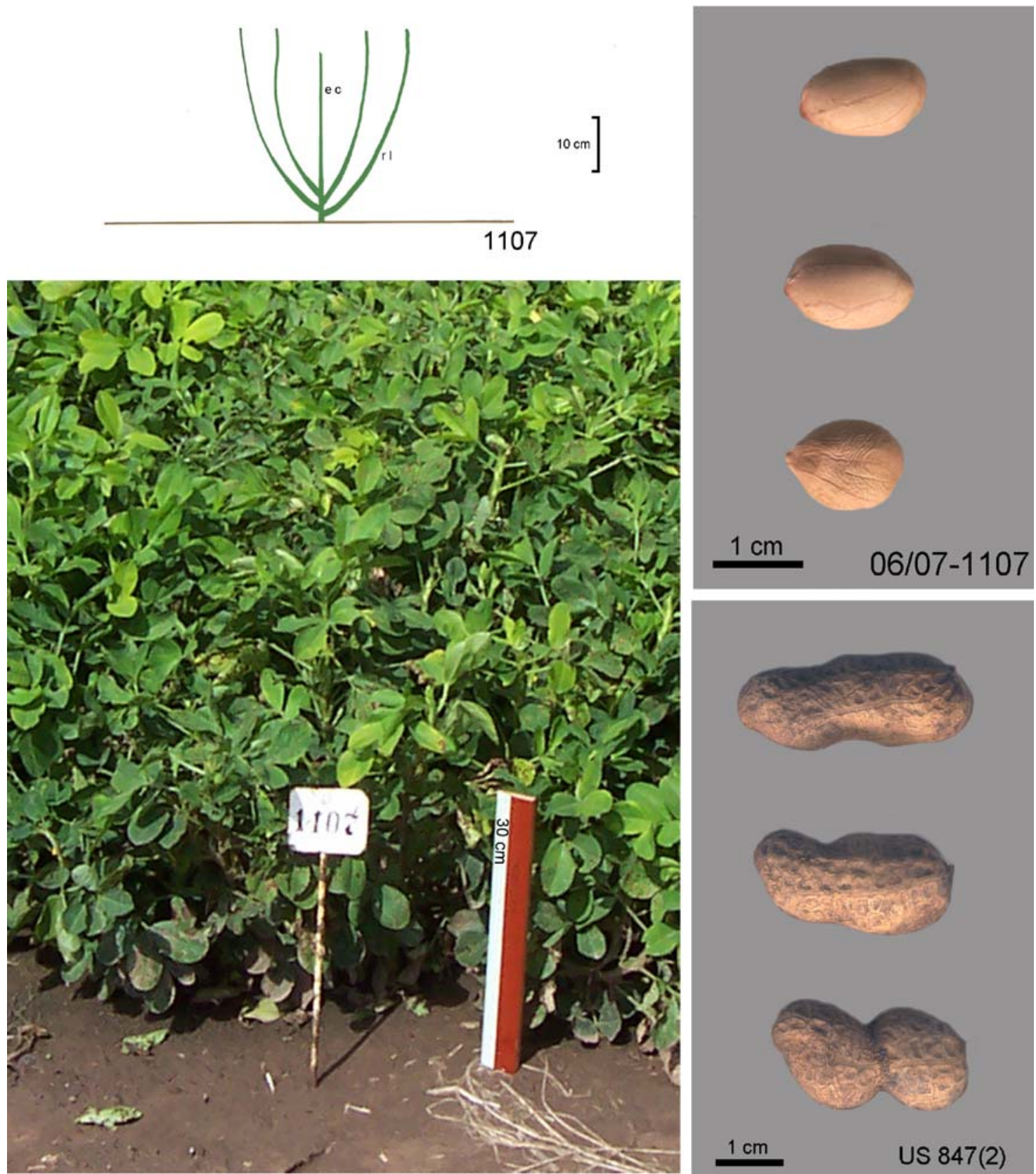


\section{Taba}

Planta poco ramificada, (4 ramas) Reproductivas alargadas. Clavo verde.

Cajas grandes, con venas longitudinales muy maarcadas, algo de pico, 3 (4) granos pálidos.
06/07 1109
$89 / 902054$
846 Wi. Taba (Yaminahua) Pando, Cobija
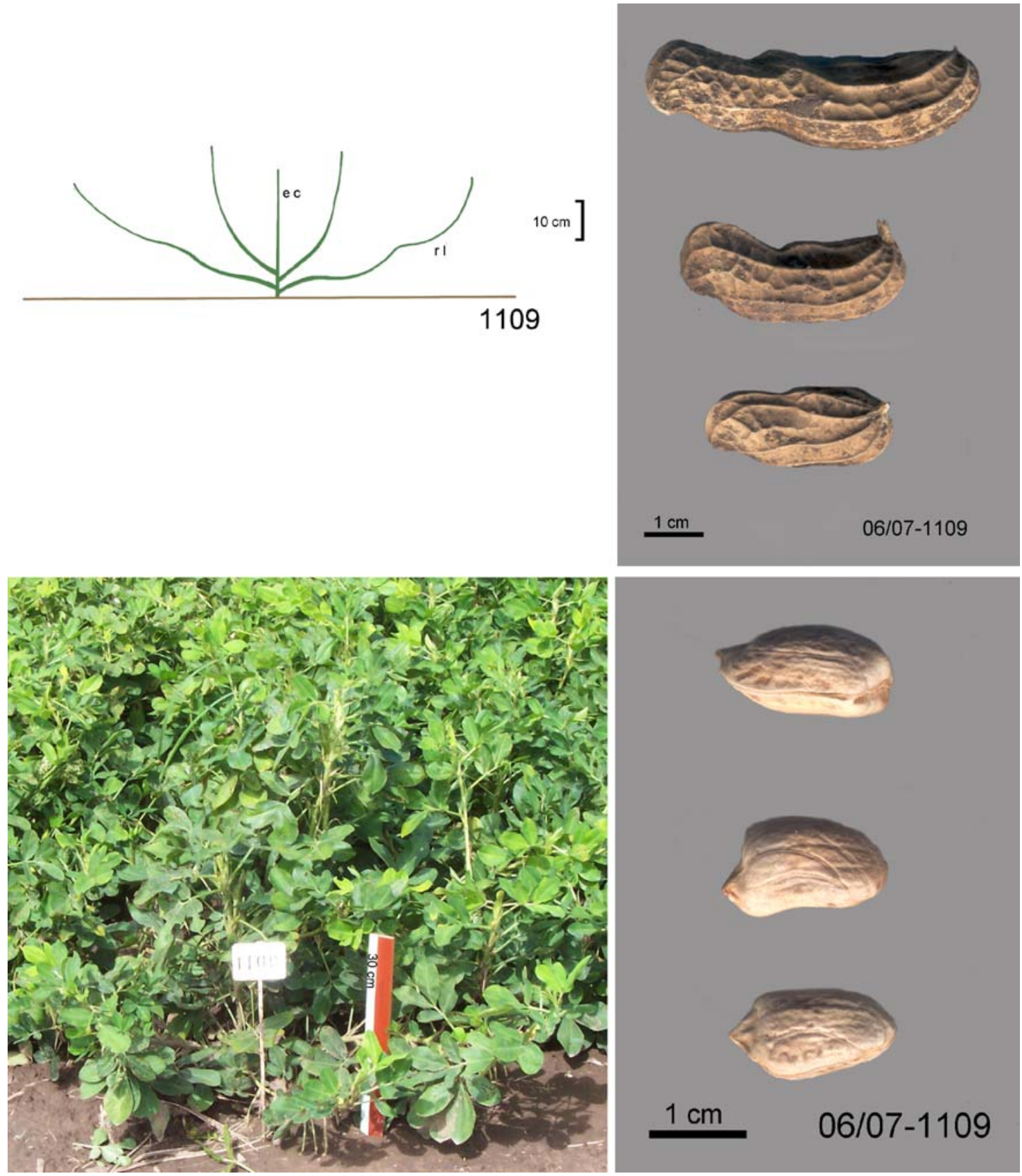


\section{Panza}

Planta poco ramificada, abierta. Reproductivas largas. Tallo violáceo, brote verde. Clavos violáceaos. Con abundantes cerdas.

Cajas con venas longitudinal del retículo muy marcado, algo de constricción., 3-4 granos violáceaos.
06/07 1110
$85 / 862532$
1111
$89 / 902057$
US $607 \mathrm{PZi}$
$762 \mathrm{Wi}$
Beni, Rurrenabaque
mani panza La Paz, Tumupasa
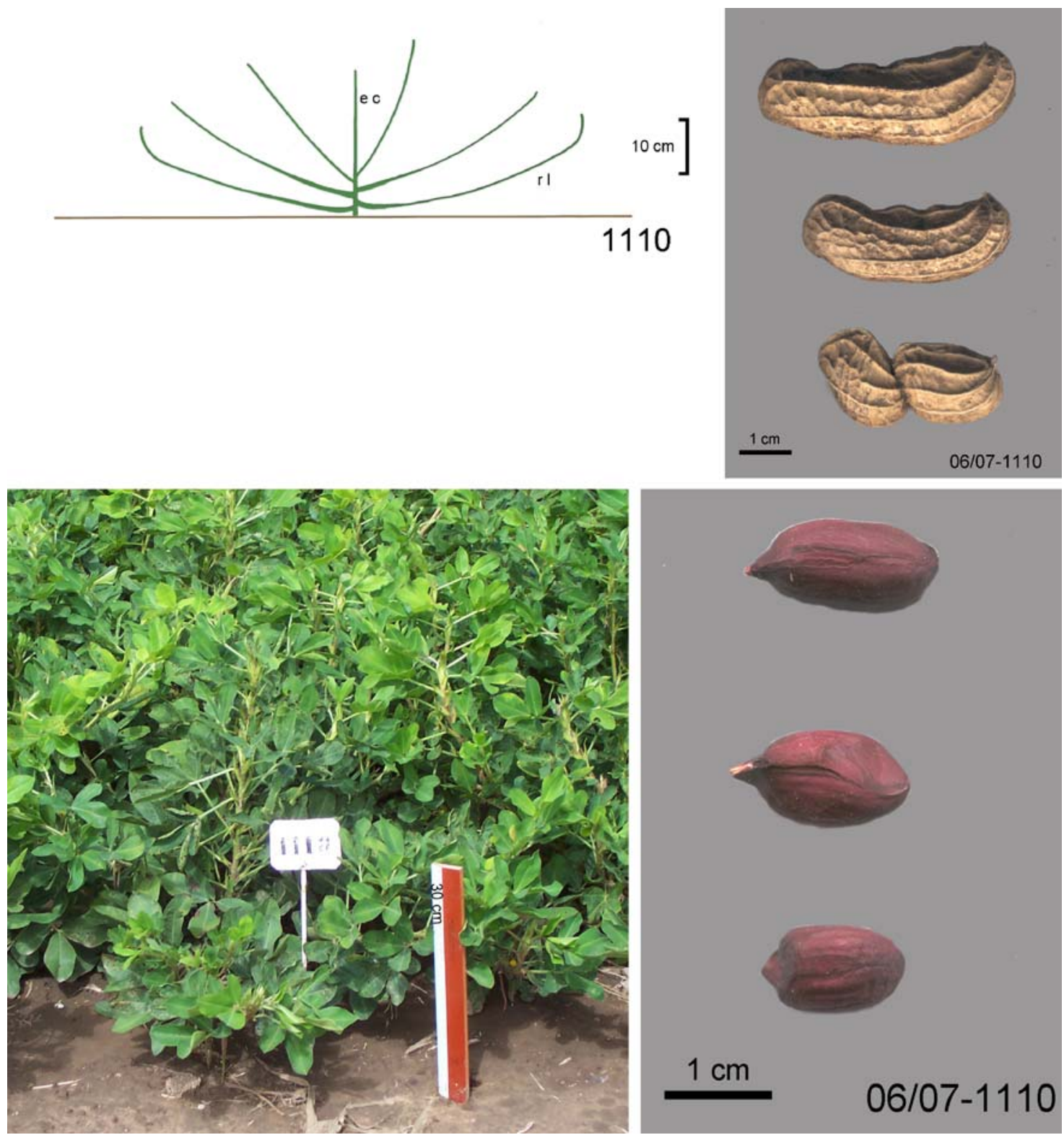

85/86 2533

$\begin{array}{rrr}\text { US } & 608 & \text { PZi } \\ 1001 & \text { Wi }\end{array}$

Beni, Rurrenabaque

89/90 2218 
Krapovickas, A.\& al., Las razas de maní de Bolivia

\section{Agradecimientos}

Los aportes de David E. Williams a este trabajo fueron apoyados por el Jardín Botánico de Nueva York, el Departamento de Agricultura de los Estados Unidos, la Fundación Fulbright, la Organización de Estados Americanos, y Bioversity International. David E. Williams también agradece a la doctora Devra Jarvis por haber propuesto esa publicación para el apoyo de la Agencia Suiza para el Desarrollo y la Cooperación, como parte de sus iniciativas globales de conservación in situ de la agrobiodivesidad y la estandarización de metodologías.

\section{Bibliografía}

ANÓNIMO. 1954. La marché mondial des arachides. Pags. 1060-1101.

GREGORY, W. C. 1977. The collection of peanut germplasm 1976-1977. Technical Report to the IBPGR. MSS, 118 págs.

—. 1979. IBPGR Technical Report, Peanut germplasm, September 1, 1978-August 31, 1979. MSS, 25 págs.

GROSSO, N. R., C.A.GUZMÁN \& J.R. PIETRARELLI. 1988. Separación electroforética de proteínas en 65 poblaciones de maní (Arachis hypogaeea L.) de origen boliviano. Su relación taxonómica. Revista Agropecuaria de Manfredi y Marcos Juarez 4: 5-15.

—, A. KRAPOVICKAS, J.R. PIETRARELLI \& C. A. GUZMÁN. 1994 Las proteínas seminales del maní (Arachis hypogaea, Leguminosae) y su relación con las categorías infraespecíficas. Bonplandia 8(1-4): 221-233.

KRAPOVICKAS, A. 2004. Consideraciones prehistóricas sobre el origen del maní cultivado. Academia Nacional de Agronomía y Veterinaria, t. LVIII: 320-331.

— \& W. C. GREGORY. 1994. Taxonomía del género Arachis (Leguminosae). Bonplandia 8(1-4): 1-186.

- 2007. Taxonomy of the genus Arachis (Leguminosae). Traslated by D. E. Williams and C. E. Simpson. Bonplandia 16. (Suplemento): 1-205.

— \& V. A. RIGONI. 1960. La nomenclatura de las subespecies y variedades de Arachis hypogaea L. Revista Invest. Agric. 14(2): 197-228.

MUÑOZ REYES, J. 1980. Geografía de Bolivia. Edit. Juventud, La Paz, Bolivia.

PIETRARELLI, J. R. 1961. Informe sobre un viaje realizado por la provincia de Corrientes (Argentina), Paraguay, Brasil y Bolivia para coleccionar maníes cultivados y silvestres. INTA, Serie. Informe de Técnicos No 30. Buenos Aires. 19 p.

—. 1968. Nuevo viaje de exploración para coleccionar maníes silvestres y cultivados. Boletín Informativo Manisero INTA-IADO (Manfredi) 3(12): 6. . 1976. Catálogo analítico de poblaciones de maní
(Arachis hypogaea L.), material coleccionado en las regiones guaraníticas, boliviana y de Goiás-Minas Gerais. INTA, EEA Manfredi (Córdoba). Información Técnica No 69. 29 pags.

—. 1977/78. Colección abierta maní. Números 760 al 1489. Estación Experimental Agropecuaria INTA, Manfredi, manuscrito, 10 pags.

. 1982. Prospecciones en maní (silvestres y cultivados) con participación de técnicos de la Estación Experimental Agropecuaria de Manfredi (Provincia de Córdoba) Boletín Interno de la coordinación del Programa de Oleaginosas del INTA «OLEICO» Nº17: 37-46.INTA, Manfredi.

\& A. KRPOVICKAS. 1990. Catálogo semianalítico de los maníes cultivados (Arachis hypogaea L.) originarios de la región andina (Bolivia, Perú y Ecuador), conservados en la Estación Experimental Agropecuaria de Manfredi (Provincia de Córdoba)-R. Argentina. 32pp. INTA, Manfredi.

SEIJO, J.G., G.L. LAVIA, A. FERNÁNDEZ, A. KRAPOVICKAS, D. DUCASSE \& E.A. MOSCONE. 2004. Physical mapping of the 5S and 18S-25Sr RNA genes by FISH as evidence that Arachis duranensis and A. ipaensis are the wild diploid progenitors of A.hypogaea (Leguminosae). Amer. J.Bot. 91(9): 1294-1303.

SIMPSON, C.E. 1980. The collection of peanut germplasm 1980.Argentina, Bolivia, Peru. Technical report to the IBPGR, Argentina, Bolivia, Perú. MSS 65 págs.

1982. The collection of Arachis germplasm 1981-82. Technical Report to IBPGR, Bolivia, Argentina, Brazil, MSS 32 págs.

— 1984. The collection of Arachis germplasm 1983.Argentina, Bolivia, Brasil. Technical Report to the IBPGR. MSS, 38 págs.

— \& D. L. HIGGINS. 1984. Catalog of Arachis germplasm collections in South America, 1976-1983. Texas Agricultural Experiment Station. The Texas A\&M University, College Station, Texas, USA.

WILLIAMS, D. E. 1989a. Collection of germplasm with emphasis on Arachis hypogaea - Bolivia 1988. Technical Report to the Agricultural Research Service, United States Department of Agriculture. MSS, 44 pags.

— 1989b. Exploration of Amazonian Bolivia yelds rare peanut landraces. Diversity 5(4): 12-13.

_ 1991a. Peanuts and Peanut Farmers of the Rio Beni: Traditional Crop genetic Resource Management in the Bolivian Amazon. PhD dissertation, City University of New York. University Microfilms International, number 9207138. 170 pags.

_ 1991b. Ethnobotanical evidence for the Bolivian origin of the Valencia peanut. Proceedings of the American Peanut Research and Education Society 23: 22 (resumen). . 1996. Aboriginal farming system provides clues to peanut evolution. En: Pickersgill and J. M. Lock (editores). Advances in Legume Systematic 8: Legumes of Economic Importance, págs. 11-17, Royal Botanic Gardens, Kew. 


\section{ANEXO}

Listado cronológico de las colecciones de maní de Bolivia.

\section{ABREVIATURAS}

Mdi. Manfredi

Cat. Catálogo

hy var. hypogaea

fa var. fastigiata

pe var. peruviana

vu var. vulgaris

\section{Colección L. Nanetti, VII-1946}

Cat. 73/74 77/78 85/86

$59 \quad$ Cat. 90

$36856414291854 \mathrm{~N}$ hy Churco

Sta. Cruz, Mairana

". 2014 N hy Sopachuy colorado

Sta. Cruz, Mairana

$37156714321988 \mathrm{~N}$ hy Valluno rastrero

$37256814331956 \mathrm{~N}$ hy Sara mani

$37356914342098 \mathrm{~N}$ hy Cojin valluno

$37457014352099 \mathrm{~N}$ hy Cojin valluno

$37557114361957 \mathrm{~N}$ hy Sara mani

$37657214371958 \mathrm{~N}$ hy Sara mani Mizque

$37757314381959 \mathrm{~N}$ hy Sara mani

38257814432102 N hy S.José de Chicaludo

La Paz, N. Yungas

$38558114462234 \mathrm{~N}$ hy Overo carenado Mairana

$38658214472235 \mathrm{~N}$ hy Overo carenado ".

38758314482030 N hy Colorado S. Simón "'

38858414492031 N hy Colorado S. Simón "

38958514502107 N hy Cojín valluno "'

39058614512037 N hy Cojín valluno "’

42061214562512 N fa Pálido del Beni

La Paz S. Yungas, Asuntas

42161714571944 N hy Sopachuy salmón

\section{Colección A. Krapovickas I-1950. Cochabamba}

35555114281756 K hy Valluno rastrero

36956514301954 K hy Sara mani

37056614311955 K hy Sara mani

37857414391999 K hy Chaucha morado

37957514401899 K hy Valluno rastrero

38057614411961 K hy Sara maní
38458014452233 K hy Overo carenado

39158714522236 K hy Overo

39258814531757 K hy Cojín valluno

39358914542100 K hy Cojín valluno

41661214552459 K fa Cruceño

\section{Colección A. Krapovickas 3-I-28-II-1958}

$$
\begin{aligned}
& 61814582237 \text { K hy Overo Cochabamba } \\
& 61914592460 \mathrm{~K} \text { fa Cruceño Santa Cruz } \\
& 62014602461 \text { K fa Cruceño "' "' } \\
& 62414622038 \text { K hy Colorado S. Simón }
\end{aligned}
$$

Colección W.C. Gregory, A. Krapovickas y J.

Pietrarelli, 1959

PI Mdi Mdi. Mdi. Cat.76 85/86

Cat. 90

BOLIVIA, Santa Cruz, Roboré 1-IV-59

262090 596-1 7522240 hy Overo

BOLIVIA, Santa Cruz, Warnes 4-IV-59

262092 597-1 2862481 fa Cruceño

$262093 \quad-2 \quad 2872482$ fa Cruceño

$262094 \quad-3 \quad 2882483$ fa Cruceño 
Krapovickas, A.\& al., Las razas de maní de Bolivia

$$
\begin{array}{lrlll}
262095 & -4 & 289 & 2484 & \text { fa Cruceño } \\
262096 & -5 & 290 & 2485 & \text { fa Cruceño } \\
262097 & -6 & 291 & 2486 & \text { fa Cruceño } \\
262098 & -7 & 292 & 2487 & \text { fa Cruceño } \\
262099 & -8 & 293 & 2488 & \text { fa Cruceño } \\
262100 & -9 & 294 & 2489 & \text { fa Cruceño } \\
262101 & -10 & 295 & 2490 & \text { fa Cruceño } \\
262102 & -11 & 296 & 2491 & \text { fa Cruceño } \\
262103 & -12 & 297 & 2492 & \text { fa Cruceño } \\
262104 & -13 & 298 & 2493 & \text { fa Cruceño } \\
262105 & 598 & 299 & 2494 & \text { fa Cruceño }
\end{array}
$$

\begin{tabular}{|c|c|c|c|}
\hline 262107600 & 758 & 1758 & hy Valluno rastrero \\
\hline 262108601 & 759 & 1764 & hy Mani pico loro \\
\hline 262110603 & 301 & 249 & fa Cruceño \\
\hline
\end{tabular}

BOLIVIA, Santa Cruz, Santa Cruz de la Sierra, mercado, 5-IV-59

BOLIVIA, Santa Cruz, Estacion Experimental de los Llanos, 4-IV-59

2621116043292033 hy Colorado S. Simón

2611196123102396 fa Colorado precoz

("Tennessee Red")

Colección A. Krapovickas, L. Mroginski, A.

Fernández, V-VI-1971

BOLIVIA, Tarija, V-VI-1971

73/74 77/78 85/86

150214712241 hy Overo San Telmo

150314721726 hy Crema

150414732168 hy Bayo americano Tarija

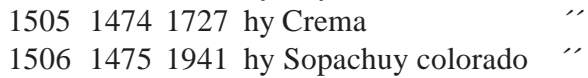

150714762242 hy Overo San Simón

150814772422 fa Cartucho Villa Montes

150914782243 hy Overo Yacuiba

151014792423 fa Cartucho

".

151114801942 hy Sopachuy salmón S.Simón

151214811943 hy Sopachuy colorado Tarija

151314822169 hy Bayo americano

151414831848 hy Churco

151614842036 hy Colorado. S. Simón Hualcalques

151714852034 hy Colorado. S. Simón

151814862244 hy Overo

151914872156 hy Bayo americano

152014882245 hy Overo Entre Ríos

152114892035 hy Colorado. S. Simón " '"

Colección W.C. Gregory, A. Krapovickas, D.J.

Banks, C.E. Simpson, J. Pietrarelli,

A. Schinini, 1976-77

PI US Mdi.85/86

BOLIVIA, Tarija, Bermejo

4682048 85/1729 hy Crema
46820610 85/1730 hy Crema

46820711 85/1849 hy Churco

BOLIVIA, Tarija, Caiza

BOLIVIA, Tarija, Cototo

46822833 85/2437 fa Cartucho

BOLIVIA, Santa Cruz, Boyuibe

46822934 85/2262 hy Overo

BOLIVIA, Santa Cruz, Lagunillas

46823035 85/2265 hy Overo

46823136 85/2263 hy Overo

85/2273 hy Overo

85/2059 hy Colorado San Simon

BOLIVIA, Santa Cruz, Gutierrez

46823237 85/2277 hy Overo

85/2278 hy Overo

85/2060 hy Colorado San Simon

BOLIVIA, Santa Cruz, Estación Experimental

Saavedra

46823338 Overo chiquitano (small pods, red to red

85/2061 Colorado San Simon

85/2281 hy Overo cojin carenado

46823439 85/1701 Rastrero colorado grande $85 / 2287$ hy Overo

46823540 85/2290 hy Overo 85/2065 hy Colorado San Simon

46823641 85/2294 hy Overo 85/2067 hy Colorado San Simon

46823742 85/2202 hy Overo salmon

BOLIVIA, Santa Cruz, Santa Cruz de la Sierra

46823843 85/2296 hy Overo

85/2069 hy Colorado San Simon

46823944 85/2407 fa Colorado precoz

46824045 85/2408 fa Colorado precoz

85/1702 Rastrero colorado grande

85/2408 fa Colorado precoz

85/2462 fa Cruceño

85/2463 fa Cruceño

46824146 85/2297 hy Overo

85/2474 fa Cruceño

Colección D.J. Banks, J. Pietrarelli, H.O. Zurita, 1976/77

BOLIVIA, Santa Cruz, Samaipata

46824247 85/1759 hy Valluno rastrero

85/1766 hy Mani pico loro

BOLIVIA, Santa Cruz, Yerba Buena

46824348 85/2122 hy Overo valluno 
85/2301 hy Overo 85/2039 hy Sopachuy colorado 85/2040 hy Colorado San Simon 85/2109 hy Cojin valluno 85/2110 hy Cojin valluno

BOLIVIA, Santa Cruz, Agua Clara 46824449 85/1945/46 hy Sopachuy colorado

BOLIVIA, Santa Cruz, Puente de Mataral 46824550 85/1767 hy Mani pico loro 85/1965 hy Sara mani 85/2126 hy Overo valluno

46824651 85/2127 hy Overo valluno 85/2041 hy Colorado carenado

BOLIVIA, Santa Cruz, Lagunilla 46824752 85/1966 hy Sara mani 85/2006 hy Colorado revoluto 85/2128 hy Overo valluno

BOLIVIA, Santa Cruz, Mataral, Muyurina 46824853 85/2129 hy Overo valluno

BOLIVIA, Santa Cruz, Vallegrande 46824954 85/1967 hy Sara mani 46825055 85/1768 hy Mani pico loro 46825156 85/1760 hy Valluno rastrero 85/1770 Sopachuy 1770 hy Mani pico loro 85/1948 Sopachuy

46825257 85/2132 hy Overo valluno 46825358 85/1771 Sopachuy

1771 hy Mani pico loro 85/1947 Sopachuy 85/1968 hy Sara mani

BOLIVIA, Santa Cruz, Trigal, Vallegrande 46825459 85/1969 hy Sara mani 85/1970 hy Mani pico loro 85/2134 hy Overo valluno 46825560 85/2137 hy Overo valluno 46825661 85/2070 hy Colorado San Simon 85/2072 hy Colorado San Simon 85/2303 hy Overo

BOLIVIA, Santa Cruz, Cochabambita, entre Vallegrande y Cochabamba 46825762 85/1971 hy Sara mani 46825863 85/1972 hy Sara mani 46825964 85/1973 hy Sara mani 85/1974 hy Sara mani 46826065 85/2131 hy Overo valluno 46826166 85/2138 hy Overo valluno 46826267 85/1975 hy Sara mani 46826368 85/2140 hy Overo valluno
BOLIVIA, Santa Cruz, Caraparial-Samaipata 46826469 85/2042 hy Colorado San Simón 2042-1 hy Sopachuy colorado

BOLIVIA, Santa Cruz, La Tuna (Mairana) 46826570 85/2104 hy Colorado San Simon 85/2112 hy Cojín valluno 85/2141 hy Overo valluno

BOLIVIA, Santa Cruz, Monteagudo, Mairana $4682667185 / 2142$ hy Overo valluno 71 85/2159 hy Overo tarijeño 46826772 85/2466 fa Cruceño

BOLIVIA, Santa Cruz, Villa Victoria, Mairana 73 85/1906 hy Colorado caja redonda 73 85/2009 hy Salmón carenado 73 85/2074 hy Colorado San Simón

46826873 85/2113 hy Cojín valluno 73 85/2143 hy Overo valluno 46826974 85/2114 hy Cojín valluno 85/2144 hy Overo valluno

BOLIVIA, Santa Cruz Valle Abajo, Mairana 46827075 85/2116 hy Cojín valluno 85/2145 hy Overo valluno

BOLIVIA, Santa Cruz San Isidro, 220 km de Santa Cruz, BPZ 46827176 85/2147 hy Overo valluno 46827277 85/1776 hy Mani pico loro 46827479 85/1779 hy Mani pico loro

BOLIVIA, Santa Cruz Comarapa BPZ 46827580 85/1781 hy Mani pico loro

BOLIVIA, Cochabamba Tenería-Aiquile, 46827681 85/1783 hy Mani pico loro 46827782 85/1853 hy Colorado carenado 82 85/1977 hy Sara maní 46827883 85/1978 hy Sara maní

BOLIVIA, Cochabamba Mesa Rancho BPZ 468279 84A 85/1979 hy Sara maní 468280 84B " 1980 hy Sara maní 46828185 85/1981 hy Sara maní " 1982 hy Sara maní 85/1991-1 hy Morado Mesa Rancho

BOLIVIA, Cochabamba Rumicancha, BPZ 468282 86A 85/1983 hy Sara maní 468283 86B 85/2000 hy Negro de Aiquile

BOLIVIA, Cochabamba Mizque 46828487 85/1784 hy Mani pico loro 468285 88-1 85/1787 hy Mani pico loro 
Krapovickas, A.\& al., Las razas de maní de Bolivia

\begin{tabular}{rrrl}
\multicolumn{1}{c}{$88-2$} & 1857 & hy Ronco \\
468286 89 & $85 / 1795$ & hy Mani pico loro \\
& $85 / 1856$ & hy Churco \\
46828790 & $85 / 1788$ & hy Mani pico loro \\
& $85 / 1789-1$ & hy Churco \\
& 1858 & hy Churco de Tarija \\
& 1859 & hy Churco \\
& 1860 & hy Churco \\
46828891 & $85 / 1790$ & hy Mani pico loro \\
& 1791 & hy Churco \\
46828992 & $85 / 1792$ & hy Mani pico loro \\
& 1861 & hy Churco
\end{tabular}

BOLIVIA, Cochabamba Cochabamba

46829093 85/2469 fa Cruceño

46829194 85/1794 hy Mani pico loro

46829295 85/2306 hy Overo

46829396 85/1762 hy Valluno rastrero

85/1796 hy Mani pico loro

85/1797 hy Sopachuy colorado

46829497 85/1799-1 hy Ronco

85/1907 hy Valluno rastrero

85/2224-1 hy Bayo americano

2309 hy Overo

46829598 85/2015 hy Colorado carenado

85/2203 hy Overo salmón

46829699 85/1704 hy Mani pico loro

BOLIVIA, La Paz La Paz

468297100 85/2075 hy Colorado San Simón

85/2311 hy Overo

468298101 85/2076 hy Colorado San Simón

2314 hy Overo

2318 hy Overo

468299 102A 85/1915 hy Yungueño

468300 102B 85/1996 hy Negro de los Yungas

468301103 85/1731 hy Crema

1801 hy Ronco

BOLIVIA, La Paz Chulumani, S. Yungas

468303 104B 85/1918 hy Yungueño

BOLIVIA, La Paz Sanani, $1800 \mathrm{~m}$

468304105 85/1724 hy Overo veteado (se perdió)

105 85/1921 hy Yungueño

BOLIVIA, La Paz San Antonio, Chulumani, 1800 m

BPZ

468305106 85/1908 hy Yungueño

1922 hy Yungueño

2505 fa Pálido del Beni

BOLIVIA, La Paz Rancho Chico de Irupana, S.

Yungas, $2000 \mathrm{~m}$

468306 107A 85/1923 hy Yungueño

85/1909 hy Yungueño

468307 107B purple
BOLIVIA, La Paz Capani-Irupana

468308108 85/1926 hy Yungueño

BOLIVIA, La Paz Irupana

468309109 85/1927 hy Yungueño

468310 110A 85/1929 hy Yungueño

468311 110B purple or black

BOLIVIA, La Paz Chulumani BPZ

468312111 85/1930 hy Yungueño

BOLIVIA, La Paz Huancane ... BPZ

468313112 85/1935 hy Yungueño

BOLIVIA, La Paz Sacawaya, S. Yungas BPZ

468314113 85/1936 hy Yungueño 85/1997 hy Negro de los Yungas

BOLIVIA, La Paz Sirupaya, S. Yungas

468315114 85/1937 hy Yungueño

Colección W.C. Gregory, A. Krapovickas, C.E.

Simpson, A. Schinini, 1976/77

BOLIVIA, Santa Cruz San Ignacio

118 85/2321 hy Overo

Colección W.C. Gregory, A. Krapovickas, C.E. Simpson, J. Pietrarelli, A. Schinini, R.W. Gibons, 1979

Sta. Cruz, Santa Cruz

475848201 85/2454 fa Cruceño

475849202 85/2329 hy Overo

475850203 85/2409 fa Colorado precoz

2411 fa Colorado precoz

475851204 85/2331 hy Overo

475852205 85/2412 fa Colorado precoz

475853206 85/2439 fa Colorado precoz compacto

475855208 85/2332 hy Overo

475856209 85/1896 hy Valluno rastrero

475857210 85/1705 hy Rastrero colorado grande

475858211 85/1706 hy Rastrero colorado grande

Sta. Cruz, EEA Saavedra GKSPScGb

475859212 85/2455 fa Cruceño "Perla de Saavedra"

Colección W.C. Gregory, A. Krapovickas, C.E.

Simpson, J. Pietrarelli, A. Schinini, 1979

Beni, Trinidad

475860213 85/2333 hy Overo

475862215 85/1707 hy Rastrero colorado del Beni 85/1708 hy Rastrero salmón del Beni

Beni, Magdalena

475863216 85/2456 fa Cruceño 
Beni, Huacaraje

475864217 85/1720 hy Overo negro

" 2475 fa Cruceño

Beni, San Borja

475865218 85/2394 fa Colorado precoz

2416 fa Colorado precoz

2417 fa Colorado precoz

2420 fa Colorado precoz

475866219 85/2335 hy Overo

475867220 fa Pálido del Beni

Beni, San Ignacio

475868221 85/1710 hy Rastrero colorado del Beni 85/1709 hy Rastrero salmón del Beni

Beni, Guayaramerin

475869222 85/1716 hy Rastrero de Guayaramerim

Beni, Riberalta GKSPSc

475872225 85/2508 fa Pálido del Beni

Colección A. Krapovickas, C.E. Simpson, D.J. Banks, J. Pietrarelli, L. Coradín,VI-1980

$\begin{array}{ccl}\text { Potosí Villazon } & \\ 475888301 & 85 / 2336 & \text { hy Overo } \\ 475889302 & 85 / 2337 & \text { hy Overo } \\ 302-2 & 85 / 2079 & \text { hy Colorado San Simón } \\ 475890303 & 85 / 2338 & \text { hy Overo } \\ 475891304 & 85 / 2204 & \text { hy Overo carenado } \\ & 85 / 2204-1 \text { hy Overo alazán rastrero } \\ 475892305 & 85 / 2340 & \text { hy Overo } \\ 305-2 & 85 / 2080 & \text { hy Colorado San Simón } \\ 475893306 & 85 / 1863 & \text { hy Churco de Tarija } \\ 475894307 & 85 / 2341 & \text { hy Overo } \\ 475895308 & 85 / 2342 & \text { hy Overo } \\ 475896309 & 85 / 1862 & \text { hy Churco de Tarija } \\ 475897310 & 85 / 1722 & \text { hy Overo alazán rastrero } \\ 310-2 & 85 / 1733 & \text { hy Crema } \\ 475898311 & 85 / 1734 & \text { hy Crema } \\ 475899312 & 85 / 1735 & \text { hy Crema } \\ 475900313 & 85 / 2343 & \text { hy Overo } \\ 313-2 & 85 / 2081 & \text { hy Colorado San Simón } \\ 475901314 & 85 / 1736 & \text { hy Crema } \\ 475902315 & 85 / 2205-1 & \text { hy Overo rastrero } \\ 4 & 2205-2 & \text { hy Overo carenado } \\ 475903316 & 85 / 2172 & \text { hy Bayo americano } \\ 475904317 & 85 / 2173 & \text { hy Bayo americano } \\ 475905318 & 85 / 2344 & \text { hy Overo cojín carenado } \\ 318-2 & 85 / 2082 & \text { hy Colorado San Simón } \\ 475906319 & 85 / 1737 & \text { hy Crema } \\ 475907320 & 85 / 2345 & \text { hy Overo } \\ 475908321 & 85 / 1738 & \text { hy Crema } \\ 475909322 & 85 / 2083 & \text { hy Colorado San Simón } \\ 3522 & 85 / 2206 & \text { hy Overo cojín carenado }\end{array}$

$\begin{array}{rrl}475910323 & 85 / 2346 & \text { hy Overo } \\ 323-2 & 85 / 2084 & \text { hy Colorado San Simón }\end{array}$

Sta Cruz, Santa Cruz de la Sierra

475911324

475912325 85/2347 hy Overo

325-2 85/1900 hy Cojín valluno

475913326 85/2397 fa Colorado precoz

475914327 85/2399 fa Colorado precoz

2476 fa Cruceño

$475915328 \quad 85 / 2348$ hy Overo

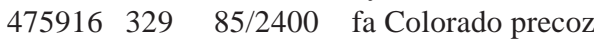

$475917330 \quad 85 / 2349$ hy Overo

330-2 85/1901 hy Cojín valluno

$47591833185 / 2477$ fa Cruceño

475920333 85/2478 fa Cruceño

475924338 85/2207 hy Overo cojín carenado

475925339 85/2479 fa Cruceño

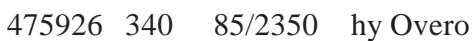

475927341 85/2402 fa Colorado precoz

475928342 85/1985 hy Cojin valluno

85/2148 hy Overo

85/2393 hy Overo

475929343

343-2 85/1902 hy Cojin valluno

475930344 85/1903 hy Cojín valluno

" 1904 hy Cojín valluno

475931345 85/2351 hy Overo

345-1 85/1994-1 hy Morado de Santa Cruz 345-2 85/1714 hy Rastrero colorado grande 345-2 85/1763 hy Rastrero colorado del

Beni

345-2 85/1994-2 hy Negro cojín

345-2 85/1994-3 hy Rastrero violáceo punta

clara

475932346 85/2117 hy Cojín valluno

475933348 85/2149 hy Overo valluno

475934349 85/1864-1 hy Churco

85/1864-2 hy Mani pico loro

Colección D.J. Banks, H.O. Zurita, L. Coradín, IV1980

\begin{tabular}{|c|c|c|c|}
\hline \multicolumn{4}{|c|}{ La Paz, La Paz } \\
\hline 475936 & 351 & 85/1739 & hy Crema \\
\hline 475937 & 352 & 85/2352 & hy Overo \\
\hline 475938 & 353 & $85 / 2353$ & hy Overo \\
\hline 475939 & 354 & $85 / 2354$ & hy Overo \\
\hline 475940 & 355 & $85 / 1740$ & hy Crema \\
\hline 475941 & 356 & $85 / 2208$ & hy Overo carenado \\
\hline 475942 & 357 & $85 / 2150$ & hy Overo valluno \\
\hline & $357-2$ & $85 / 2118$ & hy Cojin valluno \\
\hline 475943 & 358 & $85 / 2355$ & hy Overo \\
\hline 475944 & 359 & $85 / 2209$ & hy Overo cojín carenado \\
\hline 475945 & 360 & 85/1741 & hy Crema \\
\hline 475946 & 361 & 85/1742 & hy Crema \\
\hline 475947 & 362 & $85 / 2210$ & hy Overo cojín carenado \\
\hline
\end{tabular}


Krapovickas, A.\& al., Las razas de maní de Bolivia

\begin{tabular}{llll}
475948 & 363 & $85 / 1743$ & hy Crema \\
475950 & 365 & $85 / 2174$ & hy Bayo americano \\
475951 & 366 & $85 / 1744$ & hy Crema \\
475952 & 367 & $85 / 1745$ & hy Crema \\
475953 & 368 & $85 / 1746$ & hy Crema \\
475954 & 369 & $85 / 1747$ & hy Crema \\
475955 & 370 & $85 / 1748$ & hy Crema \\
475956 & 371 & $85 / 2211-1$ hy Overo carenado \\
& \multicolumn{4}{c}{$85 / 2211-2$ hy Overo rastrero } \\
475957 & 372 & $85 / 2403$ & fa Colorado precoz \\
475958 & 373 & $85 / 2356$ & hy Overo \\
& $373-2$ & $85 / 1905$ & hy Cojín valluno \\
475959 & 374 & $85 / 1749$ & hy Crema \\
475960 & 375 & $85 / 2404$ & fa Colorado precoz \\
475961 & 376 & $85 / 1750$ & hy Crema \\
475962 & 377 & $85 / 2525$ & fa Colorado picudo \\
475963 & 378 & $85 / 1751$ & hy Crema
\end{tabular}

Colección D.J. Banks, H.O. Zurita, L. Coradín, L.Janicki, IV-1980

La Paz, MACA Esperim. Station, San Pedro, Coroico 475964379 85/1939 hy Yungueño

La Paz, San Pablo, Coroico, N. Yungas 1980 m 475965380 85/1910 hy Yungueño " 85/1992 hy Morado Mesa Rancho

La Paz, Yalaka, Coroico,

475966381 85/1911 hy Yungueño

La Paz, Munaipata, Coroico

475967382 85/1912 hy Yungueño

475968383 85/2509 fa Pálido del Beni

La Paz, Coroico

475969384 85/2357 hy Overo

384-2 85/2085 hy Colorado San Simón

475970385 85/1913 hy Yungueño

La Paz, San Pedro, entre Coroico \& Caranavi, 900 m 475971386 85/1752 hy Crema

475972387

La Paz IBTA Vivero Experimental, Santa Ana 475973388

La Paz Caranavi

475974390 85/2212 hy Overo carenado

$47597539185 / 1753$ hy Crema

475976392 85/1754 hy Crema

475977393 85/2510 fa Pálido del Beni

La Paz Bella Vista, BZCJ

La Paz Santa Ana de Alto Beni, Puerto Linares.

475979397 85/1711 hy Rastrero salmón del Beni
Colección A. Krapovickas, C.E. Simpson, A.

Schinini, IV-1980

Santa Cruz, San Matías

475981399 85/2358 hy Overo

475982400 85/2359 hy Overo

Santa Cruz San José, colonia Menonita

475983401 85/2480 fa Colorado Menonitas

Santa Cruz, Natividad, Santa Cruz,

475984402 85/2360 hy Overo

\section{Colección J. Pietrarelli, R. H.Zanini, 1981}

Beni, Reyes

$\begin{array}{llll}497274 & 602-1 & \text { pink } & \\ 497275 & 602-2 & \text { red } & \\ 497276 & 602-3 & \text { tan } & \\ & 602 & 85 / 1895 & \text { hy Churco de Reyes }\end{array}$

Beni, San Buenaventura

497277603 85/2513 fa Pálido del Beni

497278 604-1 85/2514 fa Pálido del Beni

497279 604-2 85/2520 fa Colorado Rurrenabaque

Beni, Rurrenabaque

497280 605-1 85/2515 fa Pálido del Beni

497282606 85/2521 fa Colorado Rurrenabaque

497283607 85/2518 fa Negro Rurrenabaque

" 2519 fa albo

" 2532 pe Panza

607-1 85/2517 fa Pálido del Beni

607-2 85/2522 fa Colorado Rurrenabaque

608 85/2533 pe Panza

497288 608-1 85/2523 fa Colorado Rurrenabaque

Chuquisaca, Sucre, mercado

609-1 85/1802 hy Mani pico loro

610 85/1804 hy Mani pico loro

497293611 85/1806 hy Mani pico loro

612 85/1807 hy Mani pico loro

613 85/2020 hy Colorado carenado

85/2216 hy Overo cojín carenado

85/2225 hy Bayo americano

614 85/1825 hy Mani pico loro

" 85/1897 hy Valluno rastrero

614 85/2001 hy Chaucha morado

615 85/1809 hy Sopachuy salmón

615 85/1822 hy Mani pico loro

616 85/1865-1 hy Churco

"' " 2 hy Churco

85/2199 hy Overo carenado

85/2223-1 hy Bayo americano

85/2228 hy Overo alazán

617 85/1810 hy Mani pico loro 


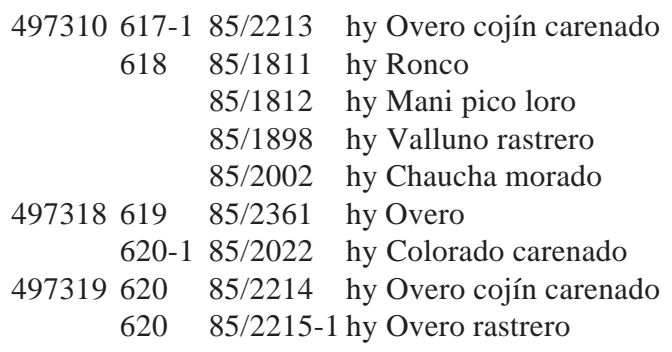

Chuquisaca, Villa Serrano, $2050 \mathrm{~m}$ 497327 623-1 85/1817 hy Mani pico loro 497328 623-2 1818 hy Ronco 497329624 85/1819 hy Mani pico loro 625 85/1820 hy Mani pico loro 497332626 85/1821 hy Mani pico loro 497334 628-1 85/1826 hy Mani pico loro 497335 628-2 1827 hy Ronco 497336 629-1 85/1830 hy Mni pico loro 497337 629-2 1831 hy Ronco 497338630 85/1832 hy Mani pico loro 631 85/1833 hy Ronco 497339 631-1 85/1834 hy Mani pico loro 497341632 85/1846 hy Mani pico loro 633 85/1835 hy Ronco 497342 633-1 85/1847 hy Mani pico loro 634 85/1836 hy Ronco 497344 634-1 85/1837 hy Mani pico loro 497346635 85/1989 hy Chaucha rosado 497347636 85/1838 hy Ronco 497348637 85/1841 hy Mani pico loro 85/2003 hy Chaucha morado

Chuquisaca, Padilla-Silliani 497349 638-1 85/1845 hy Ronco pink, pods small 638-1 1984 hy Mani pico loro 497350 638-2 85/1844 hy Ronco 497351 638-3 pink, pods large 497352 639-1 85/2004 hy Chaucha morado

Chuquisaca, Corso, $5 \mathrm{~km}$ de Tomina, camino a Sucre 497355640 85/1842 hy Ronco

\section{Colección A. Krapovickas, C.E. Simpson, A. Schinini, IV-1983}

Tarija, Bermejo 497356 805-1 85/1755 hy Crema
Tarija, Tarija

497360806

pink and white

85/1883 hy Sopachuy

85/2179 hy Bayo americano

85/1883 hy Churco

497361 807-1 85/2180 hy Bayo americano

497362 807-2 85/2362 hy Overo

497363 807-3 85/1871 hy Churco de Tarija 808-1 85/1885 hy Colorado caja redonda 497365 808-1 85/2181 hy Bayo americano 497366 808-2 85/1872 hy Churco de Tarija 497368 809-1 85/1873 hy Churco de Tarija 497370 810-1 85/1874 hy Churco de Tarija 497371 810-2 85/2182 hy Bayo americano 497372 811-1 85/1875 hy Churco de Tarija 497373 811-2 85/2153 hy Bayo americano 497374812 85/2440 fa Cartucho 497375 813-1 85/2183 hy Bayo americano 497377 814-1 85/2184 hy Bayo americano $497378 \quad-2$ 85/1888 hy Colorado caja redonda 814-2 85/2023 hy Sopachuy colorado

497381815 85/2010 hy Salmón carenado 85/1889 hy Churco de Tarija

497382 816-1 85/1876 hy Sopachuy colorado 497383 816-2 85/2185 hy Bayo americano 497385817 85/1877 hy Churco de Tarija 497387 818-1 85/2186 hy Bayo americano 497389 819-1 85/2363 hy Overo 85/2230 hy Bayo americano 497394 820-1 85/2217 hy Overo cojin carenado 497395 820-2 85/2226 hy Bayo americano 497397821 85/1878 hy Churco de Tarija

Tarija, Entre Rios

497398 822-1 85/2441 fa Cartucho 497401 823-1 85/2364 hy Overo

$497402 \quad-2$ 85/2087 hy Colorado San Simon -3 85/1891 hy Salmon carenado

497403 824-1 85/2365 hy Overo 497404 824-2 85/2088 hy Colorado San Simon 497407 825-1 85/1950 hy Sopachuy salmón 497409 826-1 85/2366 hy Overo

-1 85/2024 hy Colorado carenado 85/2218 hy Overo cojin carenado $497410 \quad-2$ 85/2089 hy Colorado San Simon 497412 827-1 85/1951 hy Sopachuy salmón 497413 827-2 85/1879 hy Sopachuy colorado 497414 828-1 85/2367 hy Overo

$497415 \quad-2$ 85/2090 hy Colorado San Simon -2 85/2529 fa Colorado dístico de Entre Ríos

$497420 \quad-4$ 85/1894 hy Sopachuy colorado

Tarija, San Simon 497417 829-1 85/2368 hy Overo

85/2219 hy Overo cojin carenado

497418 829-2 85/2091 hy Colorado San Simon 


\begin{tabular}{|c|c|c|}
\hline & $85 / 2386$ & hy Overo \\
\hline 497421 & 830-1 85/2442 & fa Cartucho \\
\hline 497422 & $830-2 \quad 85 / 2369$ & hy Overo \\
\hline 497423 & 831-1 85/2370 & hy Overo \\
\hline 497424 & 831-2 85/2092 & hy Colorado San Simon \\
\hline \multicolumn{3}{|c|}{ Tarija, Entre Rios } \\
\hline & 832-b 85/1892 & hy Sopachuy salmón \\
\hline & -f $85 / 1893$ & hy Churco \\
\hline 497426 & 832-1 85/1952 & hy Sopachuy salmón \\
\hline 497427 & $832-2 \quad 85 / 2187$ & hy Overo alazan \\
\hline 497428 & $832-3 \quad 85 / 2371$ & hy Overo \\
\hline 497429 & 832-4 85/2093 & hy Colorado San Simón \\
\hline 497430 & $833-1 \quad 85 / 2372$ & hy Overo \\
\hline 497431 & $-285 / 2094$ & hy Colorado San Simon \\
\hline \multirow[t]{3}{*}{497432} & $-385 / 1953$ & hy Sopachuy salmón \\
\hline & $-485 / 2163$ & hy Bayo americano \\
\hline & $-585 / 2196-2$ & hy Overo colorado carenad \\
\hline 497433 & 834-1 85/2188 & hy Bayo americano \\
\hline 497438 & $835-185 / 2373$ & hy Overo \\
\hline 497441 & 836-1 85/1843 & hy Mani pico loro \\
\hline 497444 & 837-1 85/2374 & hy Overo \\
\hline \multicolumn{3}{|c|}{ Tarija, Puerto Margarita, rio Pilcomayo KSSc } \\
\hline 497447 & $838 \quad 85 / 244$ & 3 fa Cartucho \\
\hline \multicolumn{3}{|c|}{ Tarija, Villa Montes } \\
\hline 497448 & $839-185 / 2444$ & fa Cartucho \\
\hline \multirow[t]{3}{*}{497450} & 840-1 85/2375 & hy Overo \\
\hline & $840-185 / 2220$ & hy Overo cojin carenado \\
\hline & $-185 / 2227$ & hy Overo carenado \\
\hline
\end{tabular}

\section{Coleccion D.E. Williams, IX-XI-1988}

Santa Cruz, prov. Ñuflo de Chávez

Mdi Mdi Mdi

DEW 88/89 1988 89/90

$\begin{array}{llll}698 & 2224 & 885 & 1980 \\ 700 & 2225 & 886 & 1981 \\ 703 & 2226 & 887 & 1982\end{array}$

hy Overo

hy Overo

hy Overo

Beni, prov. Moxos

$705 \quad 2227888 \quad 1502$

$\begin{array}{lll}710 & 2228 & 888 \\ 1983\end{array}$

hy Barcino

hy Overo

Beni, prov. Yacuma

$\begin{array}{lrrrr}721-1 & 2229 & 890 & 2013 \\ { }^{\prime} & -2 & 2230 & 891 & 2034 \\ 723 & 2231 & 892 & 2014\end{array}$

fa Colorado de Cero Ocho

fa Palido del Beni

fa Colorado de Cero Ocho

\author{
497451 840-2 85/2095 hy Colorado San Simon \\ 841 85/2221 hy Overo cojin carenado \\ 497454 841-1 85/2376 hy Overo \\ $497458 \quad 842 \quad 85 / 2445$ fa Cartucho \\ 497459 843-1 85/2446 fa Cartucho \\ 497461 844-1 85/2377 hy Overo \\ 497463 845-1 85/2447 fa Precoz de Villa Montes \\ 497465 846-1 85/2448 fa Cartucho \\ 497467 847-1 85/2449 fa Cartucho \\ -2 85/2528-1 fa Rosado palido \\ (Valencia?) \\ 497468 847-2 85/2528-2 vu Palido de Villa Montes \\ $497469848 \quad 85 / 2450$ fa Cartucho \\ 497470849 85/2451 fa Cartucho \\ $497471850 \quad 85 / 2452$ fa Cartucho \\ Tarija, Yacuiba \\ 497473 852-1 85/2378 hy Overo \\ 497475 853-1 85/2379 hy Overo \\ Tarija, Villa Montes \\ 497478 854-1 85/2453 fa Cartucho \\ 497479 854-2 85/2531 vu Palido de Villa Montes \\ 497481 855-1 85/2189 hy Bayo americano \\ 497482 855-2 85/2380 hy Overo \\ -3 85/2096 hy Colorado San Simon
}

\section{Colección Menezes, 1985}

Beni, San Borja, río Manique

s/n 85/2511 fa Pálido del Beni
Beni. Prov. Ballivian

$\begin{array}{llll}737 & 2232 & 893 & 1503\end{array}$

738-1 $2233894 \quad 2015$

"- $2 \quad 2234895 \quad 2035$

$7392235896 \quad 2016$

" $\quad 2236897 \quad 2036$ hy Rastrero salmón del Beni San Borja $14^{\circ} 50^{\prime} \mathrm{S} 66^{\circ} 47^{\prime} \mathrm{W}$ fa Colorado de Rurrenabaque Rurrenabaque $14^{\circ} 30^{\prime} \mathrm{S} 67^{\circ} 30^{\prime} \mathrm{W}$ fa Palido del Beni fa Colorado de Rurrenabaque fa Palido del Beni
Yotaú, $16^{\circ} 05^{\prime} \mathrm{S} 63^{\circ} 05^{\prime} \mathrm{W}$

Urubichá, $15^{\circ}, 20^{\prime} \mathrm{S} 62^{\circ} 55^{\prime} \mathrm{W}$

S. Pablo $15^{\circ} 40^{\prime} \mathrm{S} 63^{\circ} 15^{\prime} \mathrm{W}$

S. Ignacio de Moxos, $14^{\circ} 50^{\prime} \mathrm{S} 65^{\circ} 35^{\prime} \mathrm{W}$

Cero Ocho, $14^{\circ} 30^{\prime} \mathrm{S} 66^{\circ} 30^{\prime} \mathrm{W}$

" "

", 


$\begin{array}{cccc}740-1 & 2237 & 898 & 2017 \\ \prime "-2 & 2238 & 899 & 2037 \\ 741 & 2239 & 900 & 1504 \\ 742 & 2240 & 901 & 1984 \\ \prime \prime & 2241 & 902 & 2018 \\ 743 & 2242 & 903 & 1987 \\ \prime \prime & 2243 & 904 & 1985 \\ 746-1 & 2244 & 905 & 2019 \\ -2 & 2245 & 906 & 2038 \\ 747 & 2246 & 907 & 2039\end{array}$

La Paz, prov. Iturralde

$\begin{array}{lll}757 & 2247908 \quad 2020\end{array}$

$\begin{array}{llll}762 & 2248909 \quad 2057\end{array}$

$763 \quad 2249910 \quad 2040$

$\begin{array}{llll}766 & 2250911 \quad 2041\end{array}$

" 2250-2 2048

790-1 $2251912 \quad 2021$

" - $22252913 \quad 2042$

$795 \quad 2253914 \quad 2043$

Beni, prov. Ballivian

798-1 $2254915 \quad 2022$

" - $22255916 \quad 2044$

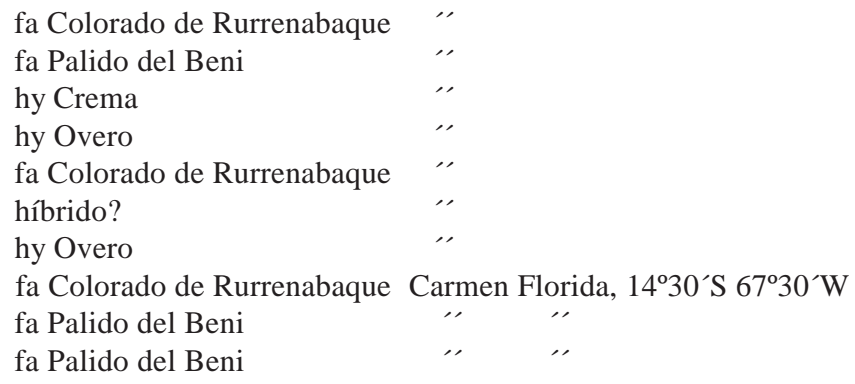

fa Colorado de Rurrenabaque S. Marcos, $13^{\circ} 30^{\prime} \mathrm{S} 67^{\circ} 25^{\prime} \mathrm{W}$ fa Palido del Beni

Pando, prov. Nicolas Suárez

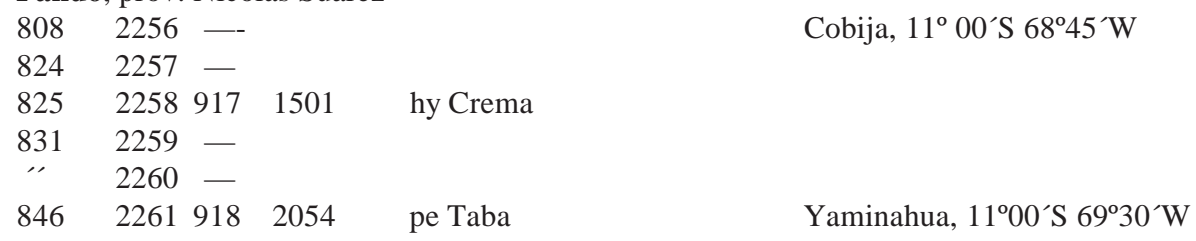

Pando, prov. Madre de Dios

85122629192023 fa Colorado de Rurrenabaque Portachuelo Bajo, $11^{\circ} 15^{\prime} \mathrm{S} 66^{\circ} 18^{\prime} \mathrm{W}$

85222639202045 fa Palido del Beni

Beni, prov. Cercado

87022649211986 hy Overo Trinidad $14^{\circ} 48^{\prime} \mathrm{S} 64^{\circ} 52^{\prime} \mathrm{W}$

Colección D.E. Williams, VI-X-1989

Sta. Cruz, Santa Cruz

9252209 fa Cruceño S.Cruz 43652

Beni, Rurrenabaque 928-1 2210

" 22211

929-1 2212

" - 22213

$930 \quad 2214$

9312215

978-1 2216-1

" - 22217

$1001 \quad 2218$

10102219

$1027 \quad 2220$
fa Colorado de Rurrenabaque 43653
fa Palido del Beni 43654
fa Palido del Beni 43655
fa Negro de Rurrenabaque 43656
fa Negro de Rurrenabaque $\quad 43657$
hy Crema 43658
fa Colorado de Rurrenabaque 43659
fa Palido del Beni 43660
pe Panza 43661
fa Colorado. Cáscara negra 43662
fa Palido del Beni 43663 
Krapovickas, A.\& al., Las razas de maní de Bolivia

$1028 \quad 2221$

fa Amarillo

43664

10342222

fa Colorado de Rurrenabaque 43665

Beni, prov. Ballivian, Carmen Florida $14^{\circ} 30^{\prime} \mathrm{S} 67^{\circ} 30^{\prime} \mathrm{W}$

1143 90/91-2627 fa Colorado. Cáscara negra

1144 90/91-2628 fa Amarillo

1146 90/91-2629 fa Palido del Beni (Pálido grande)

Beni, Rurrenabaque

$1148 \quad 2630$

hy Crema 